\title{
Unfälle mit Krafträdern im Saarland
}

Johannes Priester

Björn Wilhelm

Bernd Brutscher

Werner Knopp

Andreas Schuff

Matthias Kühn

Unfallforschung der Versicherer

Antje Lang 


\section{Unfälle mit Krafträdern im Saarland}

Dr. Johannes Priester

Björn Wilhelm

EPHK Bernd Brutscher

Prof. Dr. Werner Knopp

Dr. Andreas Schuff

Dr. Matthias Kühn

Antje Lang

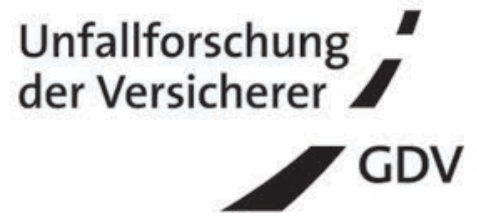




\section{Impressum}

Gesamtverband der Deutschen Versicherungswirtschaft e. V.

\section{Unfallforschung der Versicherer}

Wilhelmstraße 43/43G, 10117 Berlin

Postfach 0802 64, 10002 Berlin

E-Mail: unfallforschung@gdv.de

Internet: www.udv.de

Facebook: www.facebook.com/unfallforschung

Twitter: @unfallforschung

YouTube: www.youtube.com/unfallforschung

ISBN-Nr.: 978-3-939163-58-9

Redaktion: Dr. Matthias Kühn, Antje Lang

Bildnachweis: UDV und Ingenieurbüro Priester \& Weyde

Erschienen: 02/2015 


\section{Unfälle mit Krafträdern im Saarland}

bearbeitet durch:

Ingenieurbüro Dr. Priester

Dr. Johannes Priester

Björn Wilhelm

Priester \& Weyde

Ingenieure für forensische Unfallforschung und
-analyse in überörtlichen Bürogemeinschatten

-analyse in überörttichen
Büro Saarbrücken
Dr. Johannes Priester

Dr. Johannes Priester
Angela-Braun-Str 16

in Zusammenarbeit mit:

Landespolizeipräsidium Saarland

EPHK Bernd Brutscher

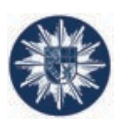

Universitätsklinikum des Saarlandes

Klinik für Unfallchirurgie Hand- und Wiederherstellungschirurgie Prof. Dr. Werner Knopp

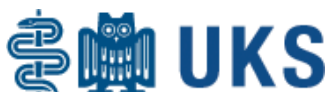

REMAKS

Rechtsmedizin am Klinikum Saarbrücken GmbH

Dr. Andreas Schuff

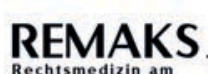

$\underset{\substack{\text { Rechnsmedizin am } \\ \text { klinikum saarbracken }}}{\text { REM }}$

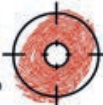

Bei der UDV betreut von:

Dr. Matthias Kühn Antje Lang

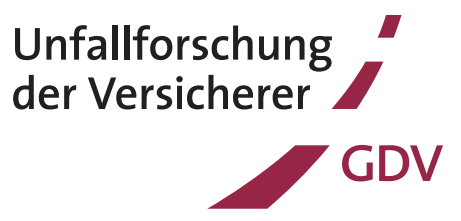





\section{Inhalt}

Inhalt. 5

Abkürzungsverzeichnis 6

Abstract 7

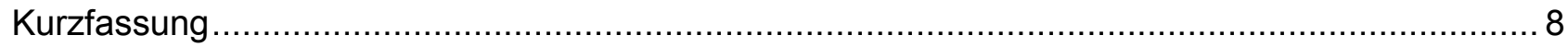

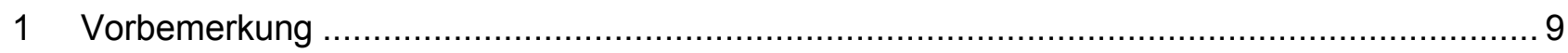

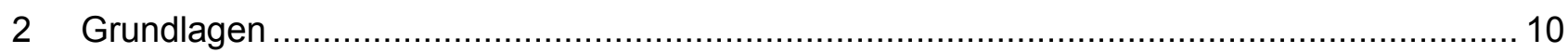

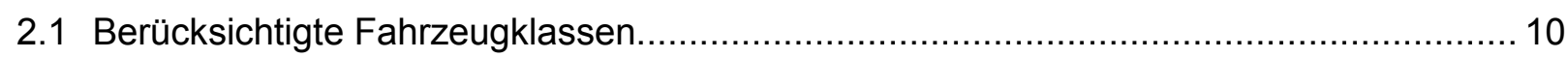

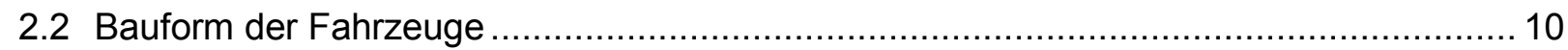

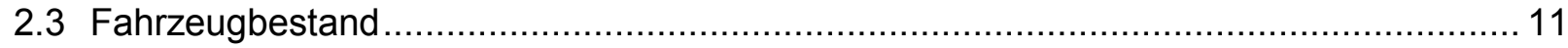

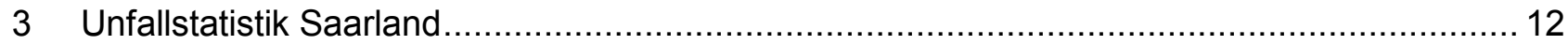

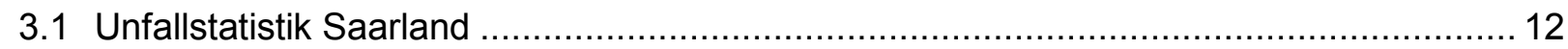

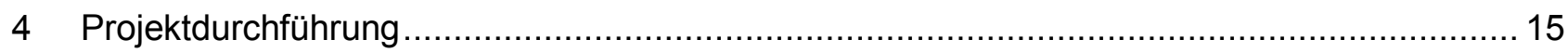

5 Vergleichbarkeit des Saarlandes als Forschungsgebiet ............................................. 16

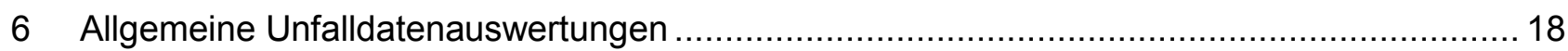

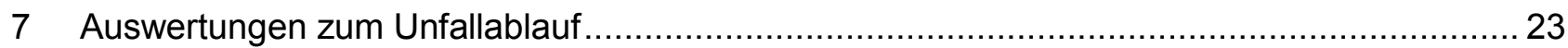

8 Auswertung unter medizinischen Gesichtspunkten ................................................. 27

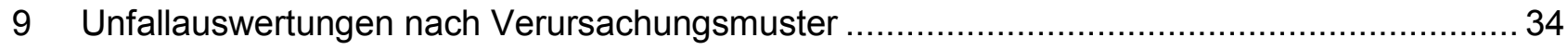

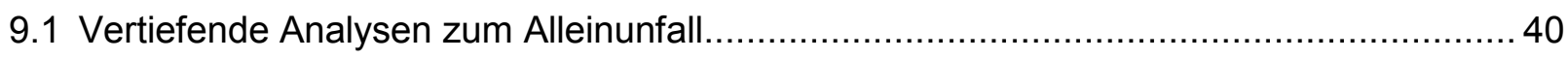

9.2 Vertiefende Analysen bei Unfallverursachung durch den Kradfahrer ............................ 42

9.3 Vertiefende Analysen bei Unfallverursachung durch den Unfallgegner.......................... 44

10 Auswertungen zu Unfällen bei schwer- bzw. schwerstverletzten Kradfahrern ..................... 46

11 Zusammenfassung der Auswertungsergebnisse ............................................................. 48

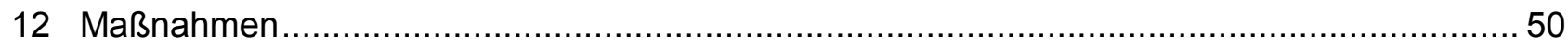

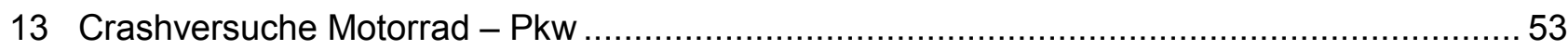

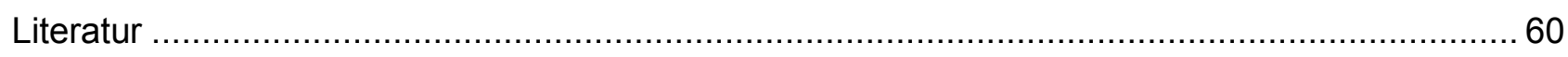

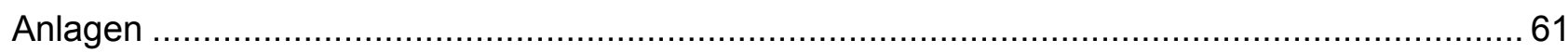




\section{Abkürzungsverzeichnis}

ABS

eCall

Pkw

Krad

kW

U

SV

LV

Get

$U(P)$

$\mathrm{U}(\mathrm{Get})$

U(SV)

U(LV)

$\mathrm{U}(\mathrm{S})$

MZR

AIS

ISO

MAIS

ISS

$\mathrm{V}_{\mathrm{A}}$

$\Delta V$

n
Antiblockiersystem

Emergency Call

Personenkraftwagen

Kraftrad

Kilowatt

Verkehrsunfall

Schwerverletzte( $r)$

Leichtverletzte( $r$ )

Getötete(r)

Unfall mit Personenschaden

Unfall mit Getöteten

Unfall mit Schwerverletzten

Unfall mit Leichtverletzten

Unfall mit Sachschaden

Motorisierte Zweiräder

Abbreviated Injury Scale

Internationale Organisation für Normung

Maximum Abbreviated Injury Scale

Injury Severity Score

Ausgangsgeschwindigkeit

Geschwindigkeitsdifferenz

Anzahl 


\section{Abstract}

According to federal statistics, in 2013 a total of 27,186 drivers and passengers of motorcycles had an accident involving injuries. Of these, 568 motorcyclists were killed because of their injury severity [1]. With a current number of about 4 million motorcycles in Germany, there is a more than an eight times higher possibility to have an accident than with a car, comparing the driven mileage of both vehicles. Considering the death rate, the risk is even about 21 times higher for motorcyclists [2].

A total of 194 traffic accidents involving motorcycles, which was assumed in advance that the motorcyclists suffered injuries, were analyzed and documented by interdisciplinary teams of researchers, such as doctors and accident analysts, during the period from May 2010 to December $31^{\text {st }}$ 2011 in the Saarland area as part of a research project.

The accidents were analyzed both technically and from a medical point of view. In particular, analysis of the accident sequence, the cause of the accident and the injuries of the people involved.

\section{Analysis of the recorded accidents}

Approximately $21 \%$ of the analyzed traffic accidents were single-vehicle accidents. If the remaining cases are considered, it should be noted that only every third accident was caused by the motorcyclist. The accident opponents were mainly cars. The accidents most frequently occurred while turning into crossroads.

Considering design and performance of the motorcycle, sports motorcycles showed aboveaverage frequency in single-vehicle accidents and self-inflicted accidents, whereas 'Tourer' and 'Choppers' were rarely involved in accidents.

Most single-vehicle accidents were caused by motorcyclists between 21 and 30 years of age. In addition, almost half of the single-vehicle accidents occurred on weekends (Saturday and Sunday).

\section{Actions}

Regular driver trainings could reduce the risk of accidents significantly. It would also be beneficial to improve the active safety for motorcycles by the use of technical assistance systems (e.g. ABS with skew detection, distance radar). Even on the roads, it is conceivable to reduce the risk of accidents by appropriate actions. Here it would be possible to significantly reduce the risk of any accident consequences for motorcyclists through the use of underride protection, attached to guardrails. Also the use of rumble strips to achieve a speed reduction on accident-prone roads would be beneficial. 


\section{Kurzfassung}

Laut Bundesstatistik verunglückten im Jahr 2013 insgesamt 27.186 Fahrzeugführer bzw. Mitfahrer von Motorrädern und zogen sich hierbei Verletzungen zu. Hiervon wurden 568 Motorradaufsassen getötet und 8974 schwer verletzt [1]. Das Risiko, bei einem Verkehrsunfall getötet zu werden, lag für Benutzer von Motorrädern bei 14 Getöteten je 100000 Krafträder. Der vergleichbare Wert für Pkw-Insassen lag mit vier Getöteten je 100000 Fahrzeuge um den Faktor 3,5 niedriger. Das bestandsbezogene Risiko auf einem Motorrad getötet zu werden, war im Jahr 2013 fast viermal so hoch wie im Auto [2].

Im Gebiet des Saarlandes wurden im Rahmen eines Forschungsprojektes insgesamt 194 Verkehrsunfälle unter der Beteiligung von Motorrädern, bei denen im Vorfeld davon auszugehen war, dass die beteiligten Motorradaufsassen Verletzungen erlitten hatten, im Zeitraum von Mai 2010 bis zum 31.12.2011 durch interdisziplinäre Forscherteams, bestehend aus Unfallanalytikern und Medizinern vor Ort aufgenommen und analysiert.

Die besichtigten Unfälle wurden sowohl aus technischer als auch aus medizinischer Sicht analysiert. Insbesondere wurden Auswertungen zum Unfallablauf, der Unfallursache sowie dem Verletzungsbild der beteiligten Personen durchgeführt.

\section{Analyse der aufgenommenen Unfälle}

Bei ca. $21 \%$ der ausgewerteten Verkehrsunfälle handelte es sich um Alleinunfälle. Werden die restlichen Fälle betrachtet, so ist festzustellen, dass lediglich jeder dritte Unfall durch den Motorradfahrer verursacht wurde. Bei den Unfallgegnern handelte es sich überwiegend um Pkws. Besonders häufig kam es zu Abbiege- bzw. Einbiegen-Kreuzen-Unfällen.

Werden Bauform sowie Leistung des Motorrades berücksichtigt, so zeigten sich sportliche Motorräder überdurchschnittlich oft bei Alleinunfällen sowie selbst verursachten Unfällen, wohingegen Tourer und Chopper eher selten an Unfällen beteiligt waren. Eine bestandsbezogene Analyse erfolgte nicht.

Die meisten Alleinunfälle wurden durch Motorradfahrer im Alter zwischen 21 und 30 Jahren verursacht. Zudem ereignete sich nahezu die Hälfte der Alleinunfälle am Wochenende (Samstag und Sonntag).

\section{Maßnahmen}

Regelmäßige Fahrertrainings könnten das Unfallrisiko deutlich senken. Zudem ist es sinnvoll, die aktive Sicherheit bei Motorrädern durch den Einsatz von technischen Assistenzsystemen (z.B. ABS mit Schräglagenerkennung, Abstandsradar) weiter zu verbessern. Auch auf den Straßen selbst ist es denkbar, die Unfallgefahr durch geeignete Maßnahmen zu reduzieren. Hier wäre es einerseits möglich, das Risiko eventueller Unfallfolgen für Motorradfahrer durch den Einsatz eines Unterfahrschutzes an Schutzplanken erheblich zu reduzieren. Andererseits wäre auch der Einsatz von Rüttelstreifen zur Temporeduzierung auf unfallträchtigen Strecken eine sinnvolle Maßnahme. 
Gegenstand des Forschungsprojektes „Unfälle mit Krafträdern im Saarland“ sind Verkehrsunfälle, die sich im Zeitraum von Mai 2010 bis einschließlich Dezember 2011 im Gebiet des Saarlandes unter der Beteiligung von Motorrädern bzw. Krafträdern ereignet haben und hierbei Personen verletzt oder getötet wurden.

Hierfür wurde mit dem Landespolizeipräsidium des Saarlandes vereinbart, dass interdisziplinäre Unfallforschungsteams zeitnah über entsprechende Unfälle informiert werden.

Im Anschluss daran wurden die gemeldeten Unfälle durch die Forschungsteams, die in der Regel aus einem Techniker bzw. Sachverständigen für Straßenverkehrsunfälle sowie einem Mediziner bestanden, möglichst zeitnah aufgesucht. An der Unfallstelle wurden die vorgefundenen relevanten Spuren sowohl aus technischer als auch medizinischer Sicht

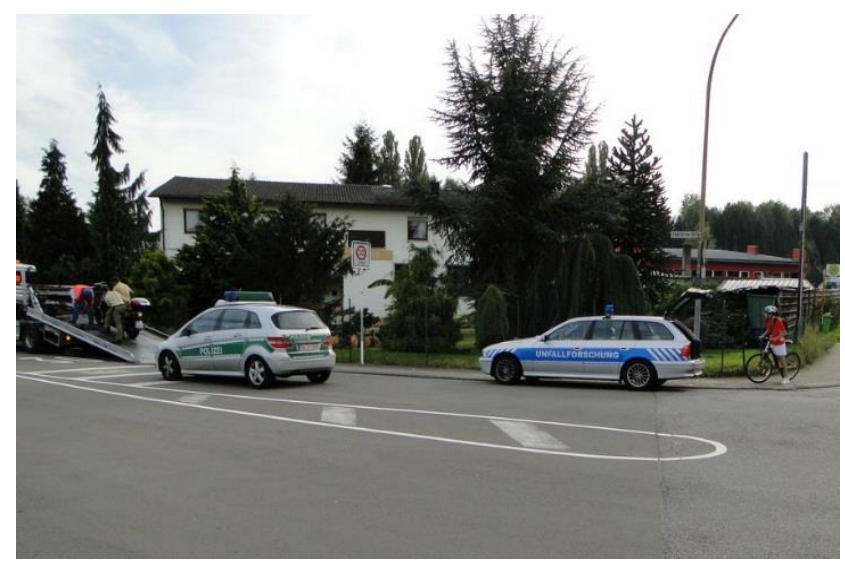

Abbildung 1:

Unfallaufnahme analysiert und dokumentiert.

In vielen Fällen konnten bereits vor Ort beispielsweise das Verletzungsbild sowie die getragenen Bekleidungsgegenstände der beteiligten Motorradaufsassen in Augenschein genommen werden. Darüber hinaus wurde auch teilweise zu den beteiligten Motorradfahrern im Nachhinein Kontakt aufgenommen, um möglichst vollständige medizinische Daten zu erhalten.

Zur weitergehenden Auswertung wurden die Unfalldaten in einer Datenbank anonymisiert erfasst. Durch die interdisziplinäre Zusammenarbeit von Medizinern und Technikern wurden in der Nachbereitung der Unfälle insbesondere Auswertungen zum möglichen Unfallablauf, der Unfallursache, zu den Verletzungen der beteiligten Personen sowie den entsprechenden Ursachen durchgeführt. Im Erhebungszeitraum wurden insgesamt 194 Motorradunfälle im Gebiet des Saarlandes untersucht. Aus den entsprechenden Ergebnissen wurden Problemstellungen sowie denkbare Maßnahmen herausgearbeitet.

Nach Auswertung der Unfalldatensätze wurde ein Pkw-Krad-Crashversuch in einer als Schwerpunkt festgestellten Kollisionskonstellation durchgeführt. 


\subsection{Berücksichtigte Fahrzeugklassen}

Im Forschungsprojekt wurden Verkehrsunfälle unter Beteiligung der folgenden von Krafträdern berücksichtigt:

- Klasse L3e: Krafträder, d. h. zweirädrige Kraftfahrzeuge ohne Beiwagen mit einem Hubraum von mehr als $50 \mathrm{~cm}^{3}$ im Falle von Verbrennungsmotoren und/oder einer bauartbedingten Höchstgeschwindigkeit von mehr als $45 \mathrm{~km} / \mathrm{h}$

- Klasse L4e: Krafträder mit Beiwagen

Allerdings gehören zu den obengenannten EG-Fahrzeugklassen [3] auch sogenannte Leichtkrafträder mit einem Hubraum von über $50 \mathrm{~cm}^{3}$ bis maximal $125 \mathrm{~cm}^{3}$ und einer Motorleistung von maximal $11 \mathrm{~kW}$ (Verhältnis Leistung/Gewicht $\leq 0,1 \mathrm{~kW} / \mathrm{kg}$ ) die nicht berücksichtigt wurden. Zur Eingrenzung der berücksichtigten Fahrzeuge oberhalb von $125 \mathrm{~cm}^{3}$ sind daher weiterhin die Fahrerlaubnisklassen [4] heranzuziehen. Zum Führen der beim Forschungsprojekt berücksichtigten Fahrzeuge sind derzeit die Fahrerlaubnisklassen A2 bzw. A erforderlich.

Die Führerscheinklasse A2 berechtigt zum Führen eines Kraftrades (auch mit Beiwagen) mit einer Motorleistung von maximal $35 \mathrm{~kW}$ bei einem Verhältnis von Leistung/Gewicht von maximal $0,2 \mathrm{~kW} / \mathrm{kg}$. Zum Erwerb der Fahrerlaubnis ist ein Mindestalter von 18 Jahren erforderlich.

Abweichend zur Klasse A2 weist die Führerscheinklasse A keine Einschränkungen im Hinblick auf Motorleistung sowie Leistungsgewicht auf. Hiermit dürfen sämtliche Krafträder (auch mit Beiwagen) geführt werden. Das Mindestalter zum Erwerb der Fahrerlaubnis beträgt für den Direkteinstieg 24 Jahre sowie bei einem Vorbesitz der Führerscheinklasse A2 von mindestens zwei Jahren 20 Jahre.

\subsection{Bauform der Fahrzeuge}

Die Krafträder werden in einer Vielzahl an Bauformen angeboten. Der Motorradmarkt bietet beispielsweise besonders sportliche Motorräder, die in der Regel voll verkleidet sind und eine hohe Motorleistung besitzen, als sogenannte Supersportler bzw. Sportler an. Eine klassische Bauform zeigt sich bei den sog. Naked-Bikes, die weitestgehend unverkleidet sind. Weiterhin findet man auch sogenannte Tourer im Straßenverkehr. Diese Motorräder sind auf Langstreckenkomfort bzw. Reisetauglichkeit ausgelegt. Diese sind von den sogenannten Sporttourern abzugrenzen, die eine tourentaugliche Sitzposition mit den

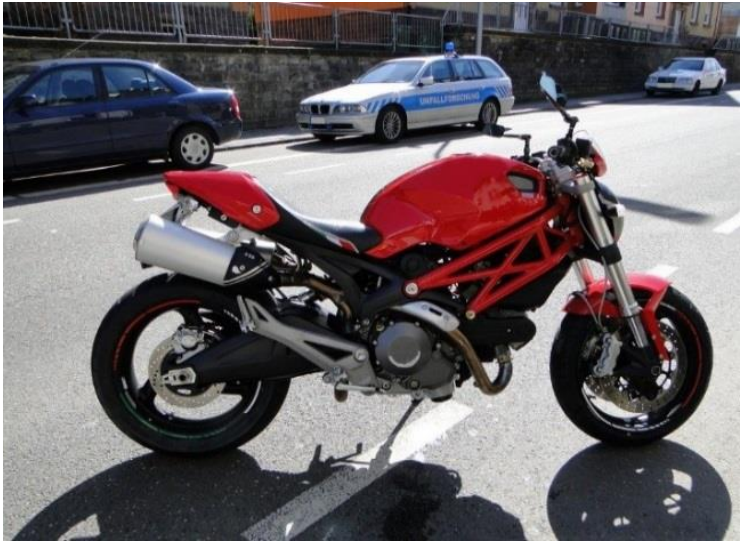

Abbildung 2:

Bauform Naked-Bike Fahrleistungen eines Sportlers kombinieren. Als Enduros werden Motorräder bezeichnet, die insbesondere auf Grund ihrer Fahrwerksgeometrie sowie Bereifungseigenschaften eine Geländegängigkeit aufweisen. Eine weitere Bauform stellt der Chopper bzw. Cruiser dar. Diese Fahrzeuge sind durch eine relativ niedrige, aufrechte Sitzposition 
charakterisiert. Darüber hinaus sind entsprechende Fahrzeuge in der Regel mit relativ breiten Lenkern ausgestattet und unverkleidet.

Zusätzlich zu den vorgenannten üblichen Krad-Bauformen befinden sich am Markt sogenannte Kraftroller. Diese besitzen im Gegensatz zur übliche Baufom keinen Knieschluss. Dies bedeutet, dass ein Durchstieg zwischen Sitzbank und Frontkarosserie vorhanden ist. Auf Grund der vorhandenen Karosserieverkleidungen bieten Kraftroller einen guten Wetterschutz. In der Regel sind entsprechende Fahrzeuge abweichend zu üblichen Krafträdern mit sog. Variomatik-Getrieben ausgestattet. Hiermit entfällt die manuelle Betätigung der Gangschaltung.

\subsection{Fahrzeugbestand}

Aus den statistischen Erhebungen des KBA [5] ergibt sich aus dem zentralen Fahrzeugregister in Deutschland ein Gesamtbestand von 52.966.819 Kraftfahrzeugen (Stand: 01.01.2014). Hiervon handelt es sich bei 3.911.830 Fahrzeugen um motorisierte Zweiräder. 821.322 dieser Fahrzeuge ist durch einen Hubraum von bis zu $125 \mathrm{~cm}^{3}$ charakterisiert.

Im Gebiet des Saarlandes ergibt sich aus den statistischen Erhebungen des KBA [6] mit Stand vom 01.01.2014 ein Gesamtbestand von 713.328 Kraftfahrzeugen. Hiervon kann der Anteil an Krafträdern mit 57.333 Fahrzeugen angegeben werden. Bei dem vorgenannten Wert sind allerdings auch Krafträder mit einem Hubraum von bis zu $125 \mathrm{~cm}^{3}$ sowie auch dreirädrige oder leichte vierrädrige Kraftfahrzeuge berücksichtigt. 


\section{$3 \quad$ Unfallstatistik Saarland}

\subsection{Unfallstatistik Saarland}

Wird die polizeiliche Unfallstatistik des Saarlandes [7] herangezogen, so ereigneten sich im Projektzeitraum vom 01.07.2010 bis zum 31.12.2011 47.562 polizeilich registrierte Verkehrsunfälle. Wird nach dem Verletzungsgrad der beteiligten Personen unterschieden, so lässt sich der Statistik entnehmen, dass insgesamt 62 Personen getötet, 1.119 Personen schwerverletzt sowie 7.432 Personen leicht verletzt wurden.

Die entsprechenden Unfallzahlen sind in der nachfolgenden Tabelle nach Monaten dargestellt.

Tabelle 1:

Verkehrsunfälle im Saarland

Quelle: [7]

\begin{tabular}{|c|c|c|c|c|c|c|c|}
\hline & $\mathbf{U}$ & $\mathbf{U}(\mathbf{G e t})$ & $\mathbf{U}(\mathbf{S V})$ & $\mathbf{U}(\mathbf{L V})$ & $\begin{array}{c}\text { Anzahl } \\
\text { Get }\end{array}$ & $\begin{array}{c}\text { Anzahl } \\
\text { SV }\end{array}$ & $\begin{array}{c}\text { Anzahl } \\
\text { LV }\end{array}$ \\
\hline Juli 10 & 2394 & 10 & 67 & 322 & 11 & 72 & 430 \\
\hline Aug 10 & 2571 & 5 & 60 & 302 & 6 & 66 & 409 \\
\hline Sep 10 & 2621 & 1 & 48 & 368 & 1 & 50 & 482 \\
\hline Okt 10 & 2717 & 1 & 56 & 308 & 1 & 62 & 425 \\
\hline Nov 10 & 2833 & 1 & 50 & 298 & 1 & 54 & 435 \\
\hline Dez 10 & 3282 & 1 & 47 & 266 & 1 & 48 & 357 \\
\hline Jan 11 & 2621 & 3 & 42 & 256 & 3 & 52 & 345 \\
\hline Feb 11 & 2438 & 3 & 39 & 228 & 4 & 46 & 306 \\
\hline März 11 & 2430 & 5 & 51 & 231 & 5 & 59 & 337 \\
\hline Apr 11 & 2584 & 5 & 64 & 314 & 5 & 74 & 443 \\
\hline Mai 11 & 2668 & 3 & 61 & 362 & 3 & 67 & 493 \\
\hline Jun 11 & 2447 & 3 & 80 & 308 & 4 & 98 & 459 \\
\hline Juli 11 & 2275 & 2 & 53 & 284 & 2 & 56 & 391 \\
\hline Aug 11 & 2734 & 1 & 65 & 333 & 1 & 69 & 425 \\
\hline Sep 11 & 2613 & 6 & 65 & 317 & 6 & 71 & 408 \\
\hline Okt 11 & 2608 & 3 & 58 & 314 & 3 & 68 & 435 \\
\hline Nov 11 & 2742 & 3 & 52 & 298 & 3 & 57 & 432 \\
\hline Dez 11 & 2984 & 1 & 44 & 20 & 2 & 50 & 420 \\
\hline Summe & 47562 & 57 & 1002 & 5429 & 62 & 1119 & 7432 \\
\hline
\end{tabular}

Im vorgenannten Zeitraum ereigneten sich unter der Beteiligung motorisierter Zweiräder insgesamt 1.354 Unfälle. In 947 Fällen kam es hierbei zu Verletzungen der Unfallbeteiligten, bei weiteren 10 Verkehrsunfällen kam es zu Verletzungen mit Todesfolge. Wird ausschließlich das Verletzungsbild der Zweiradaufsassen betrachtet, so zeigt sich, dass im Zeitraum vom 01.07.2010 bis 31.12.2011 insgesamt 10 Zweiradaufsassen getötet, weitere 239 schwere Verletzungen erlitten und darüber hinaus 747 Zweiradaufsassen leicht verletzt wurden. 
Die Verteilung der Verkehrsunfälle unter Beteiligung von motorisierten Zweirädern ist in der nachfolgenden Tabelle nach Monaten aufgezeigt.

Tabelle 2:

Verkehrsunfälle im Saarland unter Beteiligung motorisierter Zweiräder Quelle: [7]

\begin{tabular}{|c|c|c|c|c|c|c|c|}
\hline & $\mathbf{U}$ & $\mathbf{U}(\mathbf{G e t})$ & $\mathbf{U}(\mathbf{S V})$ & $\mathbf{U}(\mathbf{L V})$ & $\begin{array}{c}\text { getötete } \\
\text { MZR- } \\
\text { Aufsassen }\end{array}$ & $\begin{array}{c}\text { schwerver- } \\
\text { letzte MZR- } \\
\text { Aufsassen }\end{array}$ & $\begin{array}{c}\text { Leichtver- } \\
\text { letzte MZR- } \\
\text { Aufsassen }\end{array}$ \\
\hline Juli 10 & 118 & 3 & 23 & 54 & 3 & 22 & 59 \\
\hline Aug 10 & 89 & 1 & 20 & 44 & 1 & 18 & 50 \\
\hline Sep 10 & 99 & 0 & 15 & 60 & 0 & 15 & 62 \\
\hline Okt 10 & 70 & 0 & 12 & 38 & 0 & 12 & 38 \\
\hline Nov 10 & 44 & 0 & 7 & 21 & 0 & 7 & 21 \\
\hline Dez 10 & 8 & 0 & 1 & 5 & 0 & 1 & 5 \\
\hline Jan 11 & 31 & 0 & 4 & 19 & 0 & 5 & 19 \\
\hline Feb 11 & 28 & 0 & 4 & 13 & 0 & 4 & 12 \\
\hline März 11 & 69 & 1 & 18 & 35 & 1 & 19 & 37 \\
\hline Apr 11 & 90 & 3 & 15 & 46 & 3 & 15 & 52 \\
\hline Mai 11 & 110 & 1 & 20 & 58 & 1 & 20 & 62 \\
\hline Jun 11 & 111 & 0 & 28 & 50 & 0 & 29 & 55 \\
\hline Juli 11 & 95 & 0 & 15 & 50 & 0 & 15 & 49 \\
\hline Aug 11 & 118 & 0 & 21 & 61 & 0 & 21 & 62 \\
\hline Sep 11 & 117 & 1 & 16 & 67 & 1 & 15 & 75 \\
\hline Okt 11 & 82 & 0 & 13 & 44 & 0 & 13 & 47 \\
\hline Nov 11 & 45 & 0 & 6 & 23 & 0 & 5 & 25 \\
\hline Dez 11 & 30 & 0 & 3 & 18 & 0 & 3 & 17 \\
\hline Summe & 1354 & 10 & 241 & 706 & 10 & 239 & 747 \\
\hline
\end{tabular}

Bei weiterer Unterscheidung zwischen den unfallbeteiligten motorisierten Zweirädern nach Versicherungskennzeichen (Kleinkrafträder 25/45 km/h bzw. $50 \mathrm{~km} / \mathrm{h}$ ) und amtlichen Kennzeichen (Leichtkrafträder, übliche Krafträder) ergibt sich eine Verteilung der Unfälle nach Monaten, wie diese in den Tabellen 3 und 4 dargestellt ist.

Tabelle 3:

Verkehrsunfälle unter Beteiligung von motorisierten Zweirädern mit Versicherungskennzeichen Quelle: [7]

\begin{tabular}{|c|c|c|c|c|c|c|}
\hline & $\mathbf{U}$ & $\mathbf{U}(\mathbf{P})$ & $\mathbf{U}(\mathbf{S})$ & $\begin{array}{c}\text { getötete MZR- } \\
\text { Aufsassen }\end{array}$ & $\begin{array}{c}\text { Schwerver- } \\
\text { letzte MZR- } \\
\text { Aufsassen }\end{array}$ & $\begin{array}{c}\text { Leichtver- } \\
\text { letzte MZR- } \\
\text { Aufsassen }\end{array}$ \\
\hline Juli 10 & 45 & 32 & 13 & 1 & 6 & 26 \\
\hline Aug 10 & 39 & 26 & 13 & 0 & 3 & 23 \\
\hline Sep 10 & 41 & 28 & 13 & 0 & 2 & 26 \\
\hline Okt 10 & 35 & 20 & 15 & 0 & 3 & 17 \\
\hline Nov 10 & 26 & 15 & 11 & 0 & 4 & 11 \\
\hline
\end{tabular}




\begin{tabular}{|c|c|c|c|c|c|c|}
\hline Dez 10 & 7 & 6 & 1 & 0 & 1 & 5 \\
\hline Jan 11 & 16 & 12 & 4 & 0 & 1 & 11 \\
\hline Feb 11 & 14 & 7 & 7 & 0 & 2 & 6 \\
\hline März 11 & 30 & 22 & 8 & 0 & 3 & 20 \\
\hline Apr 11 & 28 & 20 & 8 & 1 & 3 & 19 \\
\hline Mai 11 & 28 & 20 & 8 & 0 & 1 & 20 \\
\hline Jun 11 & 35 & 24 & 11 & 0 & 5 & 22 \\
\hline Juli 11 & 45 & 30 & 15 & 0 & 4 & 21 \\
\hline Aug 11 & 42 & 25 & 17 & 0 & 4 & 31 \\
\hline Sep 11 & 47 & 32 & 15 & 0 & 1 & 24 \\
\hline Okt 11 & 40 & 27 & 13 & 0 & 1 & 12 \\
\hline Nov 11 & 21 & 12 & 9 & 0 & 51 & 331 \\
\hline Dez 11 & 18 & 14 & 4 & 0 & 2 & 5 \\
\hline Summe & 557 & 372 & 185 & & 2 & \\
\hline
\end{tabular}

Tabelle 4:

Verkehrsunfälle unter Beteiligung von motorisierten Zweirädern mit amtlichen Kennzeichen Quelle: [7]

\begin{tabular}{|c|c|c|c|c|c|c|}
\hline & $\mathbf{U}$ & $\mathbf{U}(\mathbf{P})$ & $\mathbf{U}(\mathbf{S})$ & $\begin{array}{c}\text { getötete MZR- } \\
\text { Aufsassen }\end{array}$ & $\begin{array}{c}\text { schwerverletzte } \\
\text { MZR- } \\
\text { Aufsassen }\end{array}$ & $\begin{array}{c}\text { Ieichtverletzte } \\
\text { MZR- } \\
\text { Aufsassen }\end{array}$ \\
\hline Juli 10 & 74 & 49 & 25 & 2 & 16 & 33 \\
\hline Aug 10 & 50 & 39 & 11 & 1 & 15 & 27 \\
\hline Sep 10 & 58 & 47 & 11 & 0 & 13 & 36 \\
\hline Okt 10 & 35 & 30 & 5 & 0 & 3 & 21 \\
\hline Nov 10 & 18 & 13 & 5 & 0 & 0 & 10 \\
\hline Dez 10 & 1 & 0 & 1 & 0 & 4 & 0 \\
\hline Jan 11 & 15 & 11 & 4 & 0 & 3 & 8 \\
\hline Feb 11 & 14 & 10 & 4 & 0 & 17 & 6 \\
\hline Mrz 11 & 39 & 32 & 7 & 1 & 17 & 33 \\
\hline Apr 11 & 62 & 44 & 18 & 2 & 28 & 42 \\
\hline Mai 11 & 82 & 59 & 23 & 1 & 9 & 30 \\
\hline Jun 11 & 76 & 54 & 22 & 0 & 16 & 27 \\
\hline Juli 11 & 50 & 35 & 15 & 0 & 11 & 41 \\
\hline Aug 11 & 76 & 57 & 19 & 0 & 9 & 44 \\
\hline Sep 11 & 70 & 52 & 18 & 1 & 4 & 23 \\
\hline Okt 11 & 42 & 30 & 12 & 0 & 188 & 13 \\
\hline Nov 11 & 24 & 17 & 7 & 0 & & 5 \\
\hline Dez 11 & 12 & 7 & 5 & 0 & 8 & 316 \\
\hline Summe & 798 & 586 & 212 & & & \\
\hline
\end{tabular}


Es zeigt sich, dass der Anteil schwerverletzter sowie getöteter Motorradaufsassen bei motorisierten Zweirädern mit amtlichem Kennzeichen (Leichtkrafträder, Krafträder) gegenüber den motorisierten Zweirädern mit Versicherungskennzeichen (Kleinkraftrad 25/45 km/h bzw. $50 \mathrm{~km} / \mathrm{h}$ ) signifikant höher liegt. Im Zeitraum vom 01.07.2010 bis 31.12.2011 wurden bei Verkehrsunfällen unter Beteiligung von motorisierten Zweirädern mit amtlichen Kennzeichen im Saarland insgesamt 8 Motorradauf-sassen getötet, wohingegen bei Verkehrsunfällen unter Beteiligung von motorisierten Zweirädern mit Versicherungskennzeichen lediglich 2 Aufsassen tödliche Verletzungen erlitten hatten.

\section{$4 \quad$ Projektdurchführung}

Zur Realisierung einer umfassenden Unfallauswertung sowohl aus technischer als auch aus medizinischer Sicht war die Zusammenarbeit von mehreren Institutionen erforderlich. Hierbei handelte es sich im Wesentlichen um:

- Landespolizeidirektion des Saarlandes

- Universitätsklinikum des Saarlandes - Klinik für Unfallchirurgie Hand- und Wiederherstellungschirurgie

- REMAKS Rechtsmedizin am Klinikum Saarbrücken

- Ingenieurbüro Dr. Priester Saarbrücken

Vor dem Beginn der Unfallaufnahmen wurden zunächst die Erfassungsparameter festgelegt. Aus technischer Sicht wurden im Wesentlichen Erhebungen zur Auswertung der nachfolgend aufgeführten Parameter durchgeführt:

- individuelle Ausgangsparameter, z. B. Kategorisierung der Art der unfallbeteiligten Fahrzeuge, Untersuchung der beteiligten Fahrzeuge im Hinblick auf den technischen Zustand

- Parameter zur Unfallörtlichkeit, beispielsweise Ortslage, Zustand und Verlauf der Fahrbahn, Witterungsverhältnisse

- sicherheitsrelevante Parameter im Hinblick auf die beteiligten Zweiradfahrer bzw. aufsassen (z.B. Schutzkleidung ja/nein? Welche Art? Aktive Sicherheitssysteme am beteiligten Zweirad vorhanden?)

- Parameter zum Unfallablauf wie z. B. Ausgangs- und Kollisionsgeschwindigkeiten der unfallbeteiligten Fahrzeuge, vorkollisionäres Fahrverhalten, Kollisionskonstellation, postkollisionäre Auslaufbewegung

Aus medizinischer Sicht wurden allgemeine Informationen zu den Zweiradfahrern wie beispielsweise Alter, Körpergröße sowie Gewicht erhoben. Darüber hinaus wurden auch Erhebungen zur Fahrtüchtigkeit zum Unfallzeitpunkt durchgeführt. Das Verletzungsmuster der Zweiradaufsassen wurde im Hinblick auf Lage und Ausprägung detailliert dokumentiert. Hierbei wurden die Einzelverletzungen nach der Abbreviated Injury Scale (AIS98) [8] codiert. 
Mit der saarländischen Polizei wurde vereinbart, dass den Forschungsteams im Erhebungszeitraum grundsätzlich sämtliche Verkehrsunfälle unter der Beteiligung von Krafträdern zeitnah mitgeteilt werden, bei denen davon auszugehen ist, dass es sich hierbei um Verkehrsunfälle mit Personenschaden (insbesondere der Kradaufsassen) handelt. Wird nun die Zahl der polizeilicherseits erfassten Unfälle mit Krafträdern und Personenschaden im Erhebungszeitraum mit der Zahl der durch die Forschungsteams angefahrenen Unfälle verglichen, so zeigt sich, dass nicht die Gesamtanzahl der Verkehrsunfälle untersucht wurde. Dies war insbesondere darin begründet, dass eine Unfallmeldung durch die Polizei an die Forschungsteams nicht in allen Fällen erfolgte. Weiterhin war in Einzelfällen eine Anfahrt zur Unfallörtlichkeit und entsprechende Analyse durch die Forschungsteams nicht möglich.

Da allerdings keine Selektion der angefahrenen Verkehrsunfälle erfolgt ist, kann von einer stichprobenartigen Unfallaufnahme und demzufolge auch von einer Vergleichbarkeit der Unfalldaten für das Saarland ausgegangen werden.

Da nicht in allen Fällen sämtliche relevanten Parameter aus medizinischer Sicht am Unfallort erhoben werden konnten, wurde auch versucht, die Datensätze im Nachhinein zu vervollständigen. Hierzu wurde Kontakt zu den unfallbeteiligten Zweiradaufsassen aufgenommen. Die entsprechenden Personen wurden zunächst über den Postweg schriftlich kontaktiert, ob Einverständnis zu einer telefonischen Kontaktaufnahme besteht. War dies der Fall, wurde zunächst ein Telefongespräch mit den entsprechenden Personen zur weiteren Vorgehensweise geführt. Im Anschluss wurden Fragebögen zur Beantwortung relevanter Erhebungsdaten zugesandt. Die Erfassung sämtlicher medizinischer Daten in einer elektronischen Datenbank erfolgte in anonymisierter Form.

\section{$5 \quad$ Vergleichbarkeit des Saarlandes als Forschungsgebiet}

Im Hinblick auf die Ergebnisse der Unfallerhebungen im Gebiet des Saarlandes stellt sich zunächst die Frage, ob diese als vergleichbar in Bezug auf das gesamte Bundesgebiet anzusehen sind.

Wird beispielsweise die Bevölkerungsstruktur der saarländischen und gesamtdeutschen Bevölkerung im Hinblick auf Alter sowie Geschlecht verglichen, so ergeben sich lediglich geringe Abweichungen zueinander [9]. Das durchschnittliche Lebensalter ergibt sich beispielsweise im Bundesdurchschnitt mit 43,9 Jahren und weicht im Saarland mit 45,4 Jahren hiervon nur unwesentlich $\mathrm{ab}$. Auch die Verteilung der einzelnen Altersgruppen zeigt keine deutlichen Unterschiede im Vergleich zueinander.

Ein Vergleich der Gebietsstruktur des Saarlandes mit dem gesamtdeutschen Raum zeigt in beiden Fällen eine große Heterogenität verschiedener Regionen. Dies verdeutlicht insbesondere die Betrachtung entsprechender Reliefkarten. Die Flächennutzungsstruktur des Saarlandes im Vergleich mit dem gesamtdeutschen Raum verdeutlicht, dass auch hier nur geringe Abweichungen in den Kategorien „Verkehr", „Wohnen“ und „Gewerbe/Industrie“ festzustellen sind [10].

Wird die Verkehrsinfrastruktur des Saarlandes mit dem Bundesdurchschnitt verglichen, wird deutlich, dass im Saarland ein signifikant höherer Autobahnanteil (Anteil an Gesamtstraßenkilometern Saarland 11,7\%, Bundesgebiet: 5,6\%) vorliegt [11]. 
Der Anteil der verschiedenen Fahrzeugtypen im Hinblick auf den Gesamtfahrzeugbestand weist im Saarland eine ähnliche Verteilung wie im gesamten Bundesgebiet auf. Es ergibt sich insbesondere ein Anteil an Pkw im Saarland von 84,6\% und an Krafträdern von 8,0\% (Bundesgebiet: Pkw $82,8 \%$, Krafträder 7,7\%) [6].

Werden die saarländische sowie die bundesweite Verkehrsunfallstatistik miteinander verglichen, so ergeben sich auch hier insbesondere bei der Verunglückten- und Getötetenrate nur relativ geringe Abweichungen zwischen dem Bundesgebiet sowie dem Saarland [1]. In den nachfolgenden Diagrammen sind die entsprechenden Kennzahlen nach Bundesländern wiedergegeben.

\section{Verunglückte je 100.000 Einwohner}

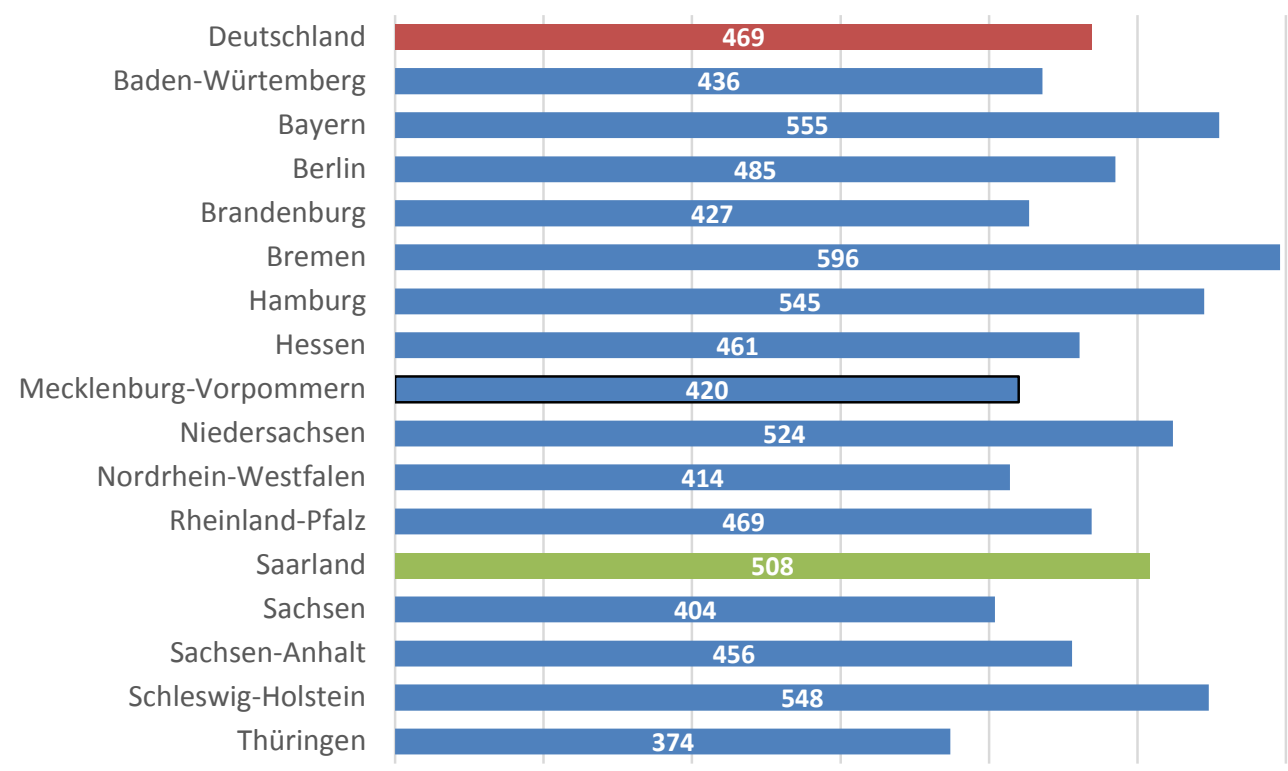

Diagramm 1:

Verunglücktenrate nach Bundesländern (2013)

Quelle: [1] 


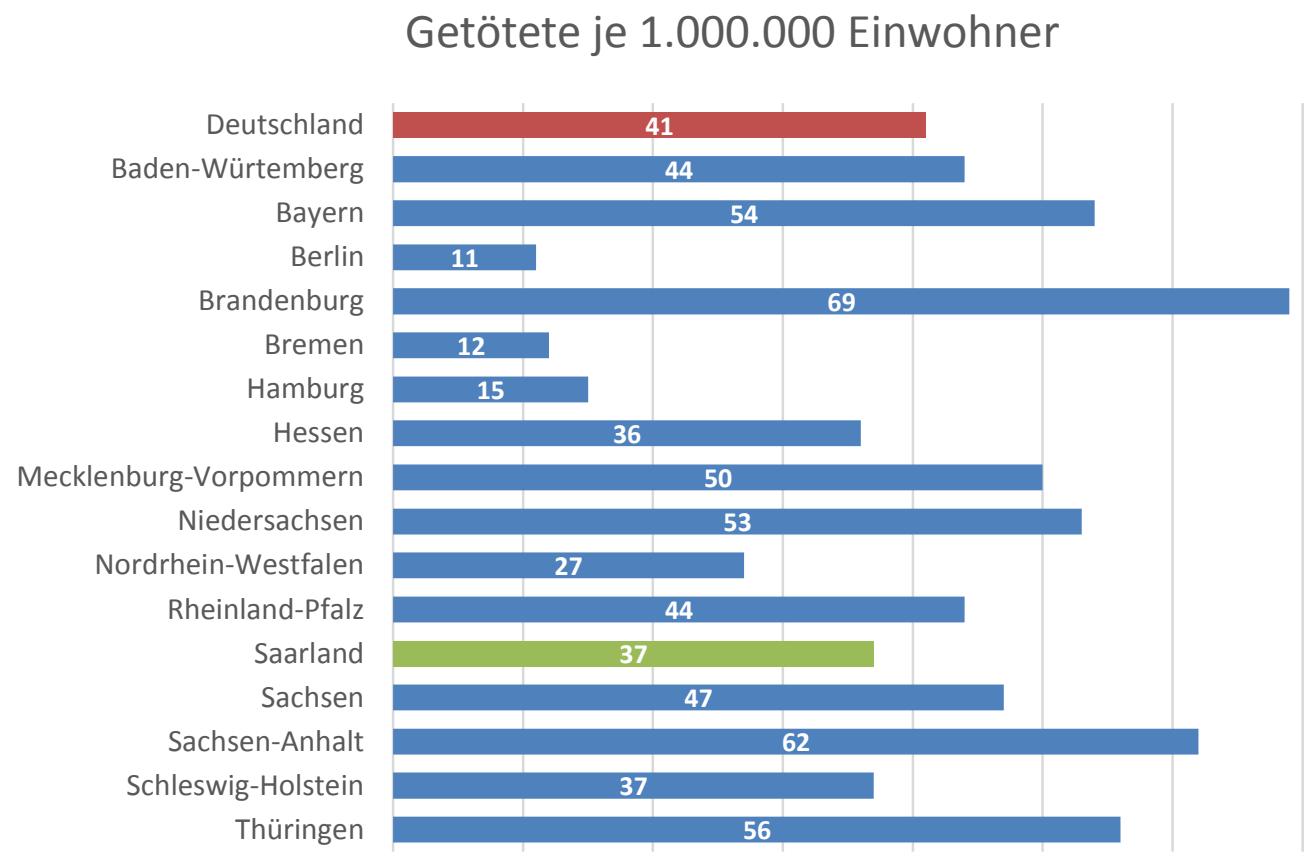

Diagramm 2:

Getötetenrate nach Bundesländern (2013)

Quelle: [1]

Zusammenfassend ist folglich bei einer Vielzahl an Merkmalen von einer guten Vergleichbarkeit der für die Unfallforschung relevanten Parameter zum gesamten Bundesgebiet auszugehen.

\section{6}

Allgemeine Unfalldatenauswertungen

Im Forschungsprojekt wurden im Zeitraum von Mai 2010 bis einschließlich Dezember $2011 \mathrm{im}$ Gebiet des Saarlandes insgesamt 194 Verkehrsunfälle unter der Beteiligung von Krafträdern vor Ort analysiert und weitergehend ausgewertet. Die wesentlichen allgemeinen Auswertungsergebnisse aller berücksichtigten Verkehrsunfälle sind nachfolgend wiedergegeben.

\section{Untersuchungsmonate}

Im Diagramm 3 ist die Verteilung aller im Erhebungszeitraum aufgenommenen Verkehrsunfälle unter der Beteiligung von Krafträdern über den jeweiligen Monat dargestellt. 


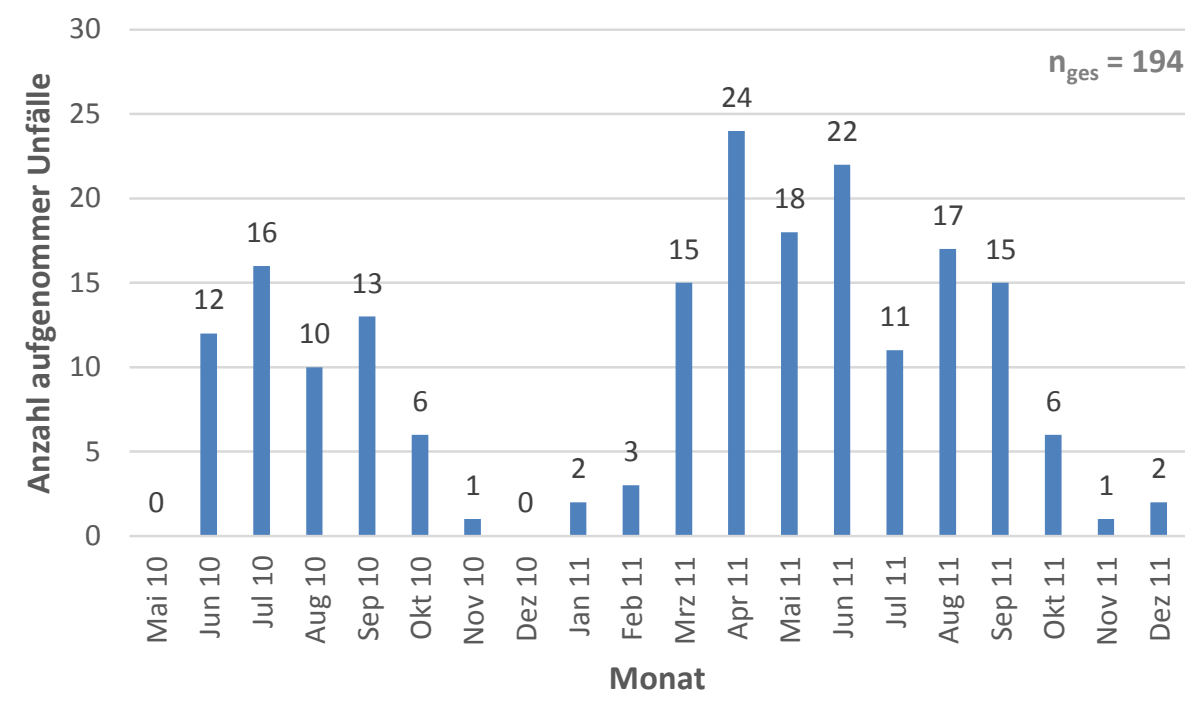

Diagramm 3:

Anzahl der untersuchten Verkehrsunfälle nach Monaten

\section{Krad-Bauform}

Im Diagramm 4 ist die prozentuale Verteilung der unfallbeteiligten Krafträder nach der jeweiligen Bauform wiedergegeben.

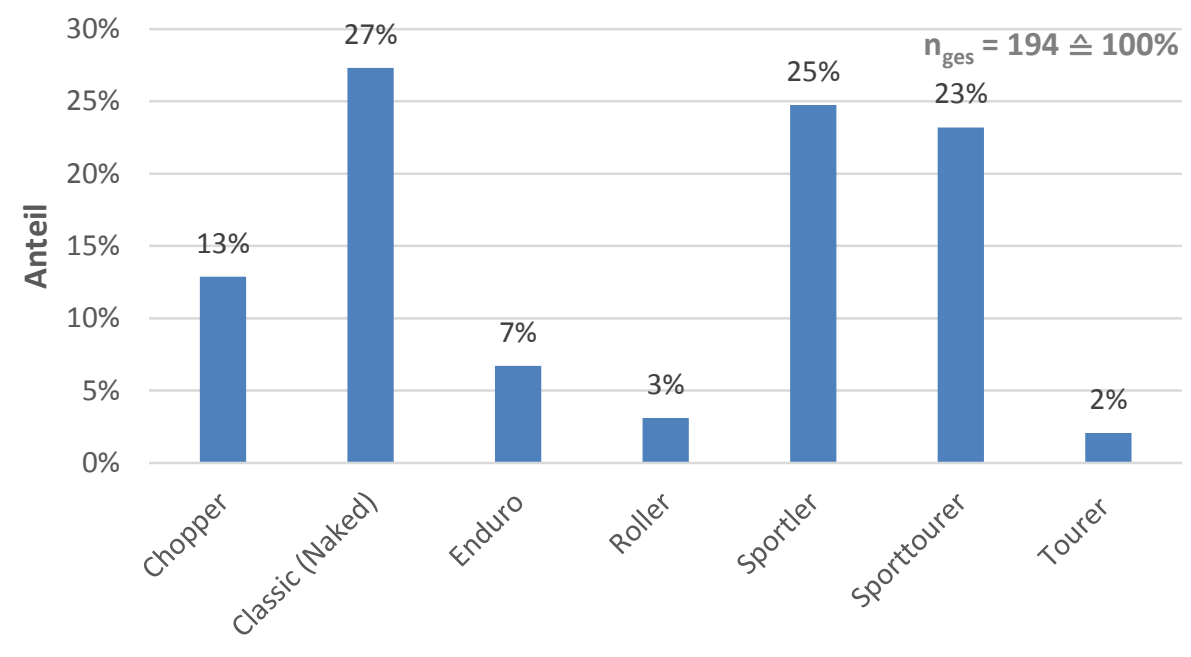

Bauform

\section{Diagramm 4:}

Prozentuale Verteilung nach Fahrzeugbauform

Es wird ersichtlich, dass es sich bei den unfallbeteiligten Krafträdern überwiegend um sogenannte Naked-Bikes bzw. Sportler sowie Sporttourer handelte. Im Hinblick auf die oben genannten Feststellungen ist allerdings darauf hinzuweisen, dass es sich hierbei lediglich um eine Auswertung der tatsächlich festgestellten Fahrzeugtypen im Rahmen der aufgenommenen Verkehrsunfälle handelt. Ein Abgleich bzw. Vergleich mit dem tatsächlichen Fahrzeugbestand erfolgte nicht. 


\section{Wochentage}

Im nachfolgend dargestellten Diagramm ist die Wochentagsverteilung aller untersuchten Verkehrsunfälle unter der Beteiligung von Krafträdern dargestellt.

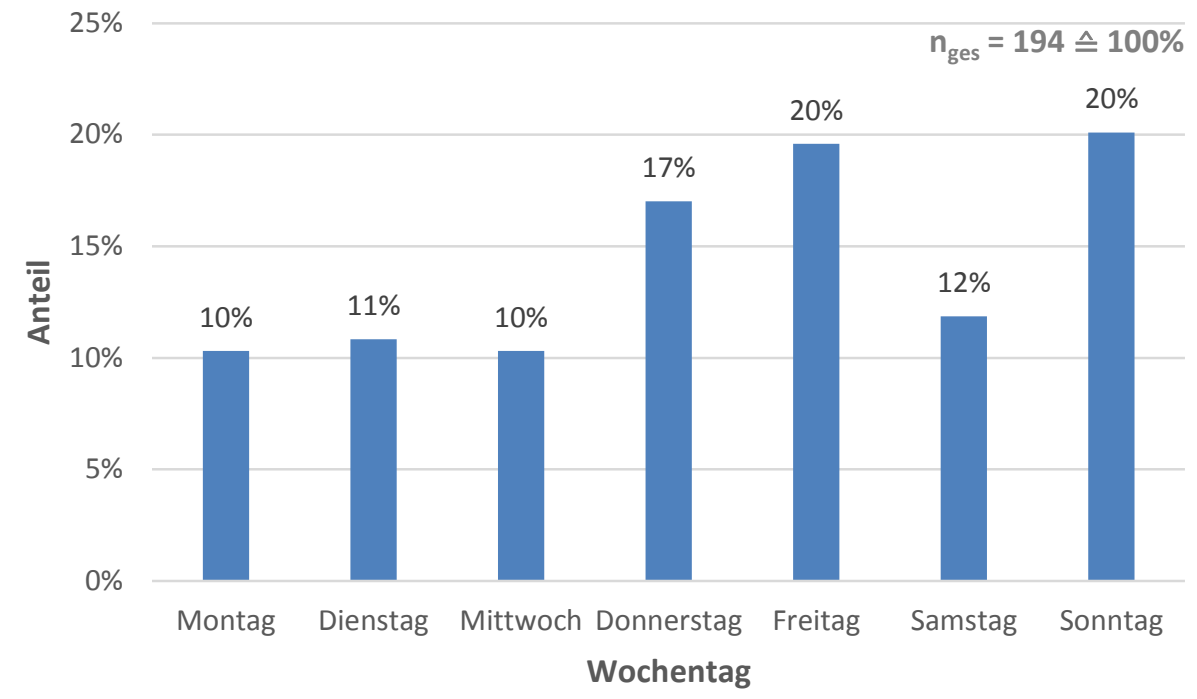

\section{Diagramm 5:}

Prozentuale Verteilung der untersuchten Verkehrsunfälle nach Wochentagen

Besonders häufig ereigneten sich Verkehrsunfälle am Wochenende (insbesondere Freitags und Sonntags).

\section{Uhrzeiten}

Die prozentuale Verteilung der von den Unfallforschungsteams untersuchten Verkehrsunfälle ist im nachfolgenden Diagramm nach Uhrzeiten dargestellt.

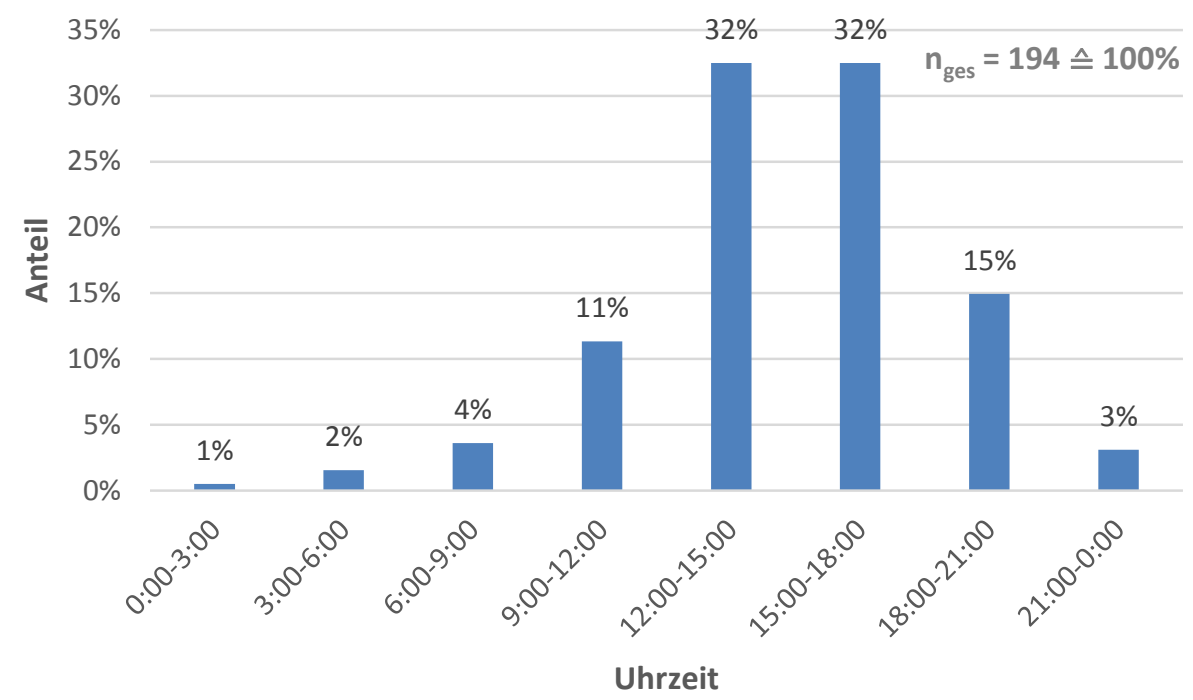

\section{Diagramm 6:}

Prozentuale Verteilung der aufgenommenen Verkehrsunfälle nach Uhrzeiten

Nahezu zwei Drittel aller analysierten Verkehrsunfälle ereigneten sich im Zeitraum von 12 Uhr bis $18 \mathrm{Uhr}$. 


\section{Ortslage}

Der größte Teil der analysierten Verkehrsunfälle (60\%) ereignete sich innerhalb geschlossener Ortschaften (siehe Diagramm 7).

\section{Ortslage}

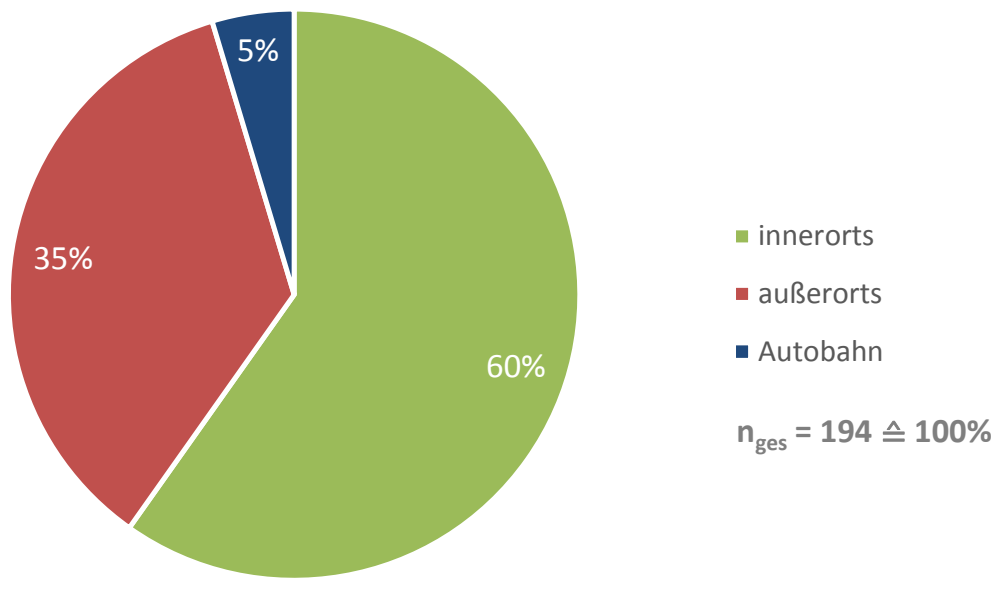

Diagramm 7:

Prozentuale Verteilung der untersuchten Verkehrsunfälle nach Ortslage

\section{Licht- und Witterungsverhältnisse}

Die prozentuale Verteilung der untersuchten Verkehrsunfälle im Hinblick auf die zum Unfallzeitpunkt herrschenden Lichtverhältnisse ist im nachfolgenden Diagramm aufgezeigt.

\section{Lichtverhältnisse}

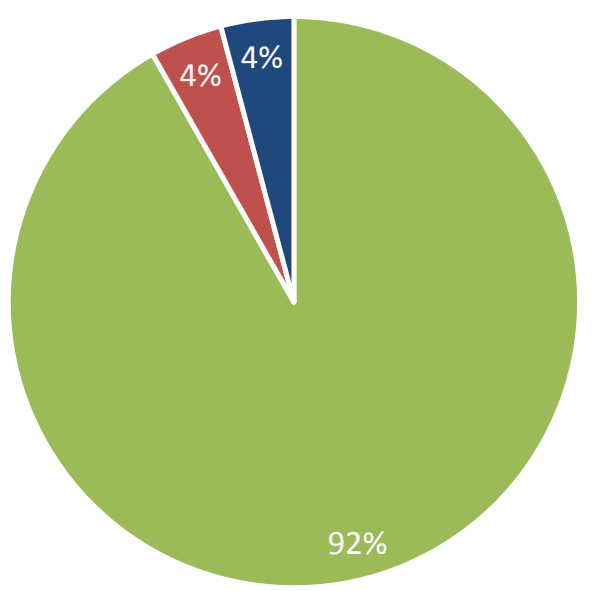

$$
\begin{aligned}
& \text { - Tageslicht } \\
& \text { - Dämmerung } \\
& \text { - Dunkelheit } \\
& \mathrm{n}_{\text {ges }}=194 \bumpeq 100 \%
\end{aligned}
$$

\section{Diagramm 8:}

Prozentuale Verteilung der untersuchten Verkehrsunfälle nach Lichtverhältnissen 
Im Hinblick auf die zum Unfallzeitpunkt herrschenden Witterungsverhältnisse ist im Diagramm 9 die prozentuale Verteilung der untersuchten Verkehrsunfälle dargestellt.

\section{Witterungsverhältnisse}

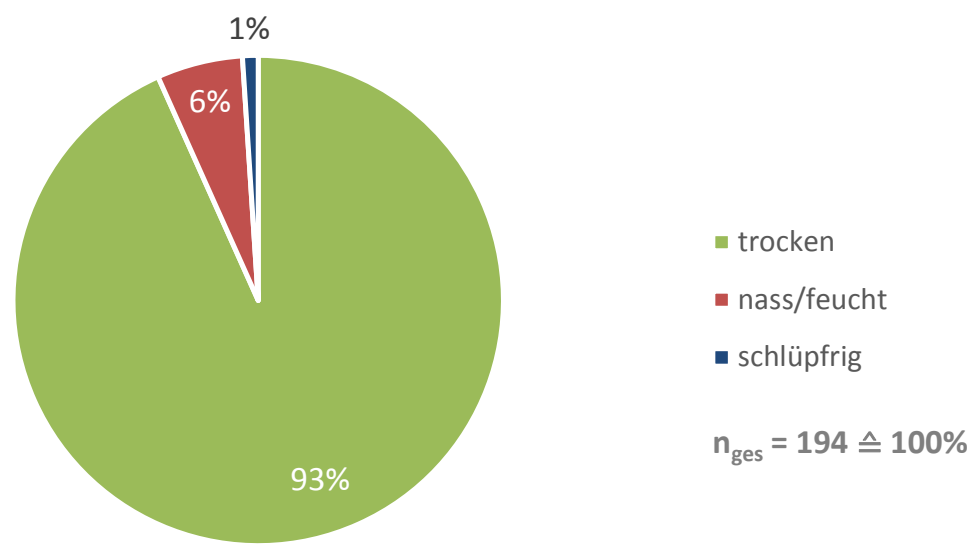

Diagramm 9:

Prozentuale Verteilung der aufgenommenen Verkehrsunfälle nach Witterungsverhältnissen

In der Regel ereigneten sich die Verkehrsunfälle bei Tageslicht und trockener Fahrbahnoberfläche.

\section{Unfallgegner}

Die prozentuale Verteilung der untersuchten Verkehrsunfälle nach Unfallgegnern ist im Diagramm 10 aufgezeigt.

\section{Unfallgegner}

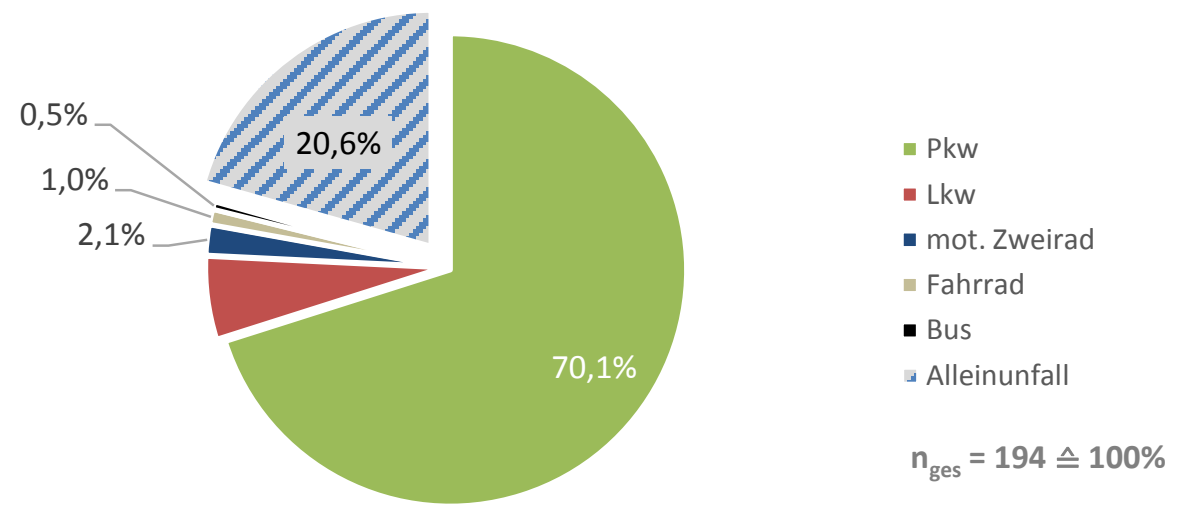

\section{Diagramm 10:}

Prozentuale Verteilung der untersuchten Zweiradunfälle nach Unfallgegnern

Nahezu 21\% der ausgewerteten Verkehrsunfälle stellten sich als sogenannte Alleinunfälle ohne die Beteiligung anderer Verkehrsteilnehmer heraus. Sofern ein Unfallgegner vorhanden war, handelte es sich bei diesem in der Regel um einen Pkw $(70,1 \%)$. 
Die Auswertungen der aufgenommenen Verkehrsunfälle im Hinblick auf den Unfallablauf erfolgten auf Grund der an der jeweiligen Unfallstelle vorgefundenen Spurenlage. Insbesondere wurden hier die Schadenbilder an den unfallbeteiligten Fahrzeugen sowie auch die unfallbedingten Spuren (z. B. Kratzspuren auf der Fahrbahnoberfläche, Endlagen bzw. Endstände der unfallbeteiligten Fahrzeuge) analysiert. Unter Zugrundelegung der vorgenannten Feststellungen wurden im Anschluss daran umfangreiche Unfallrekonstruktionen aus technischer Sicht durchgeführt. Die entsprechenden Ergebnisse sind nachfolgend im Wesentlichen wiedergegeben.

\section{Unfallverursacher}

Werden die aufgenommenen Verkehrsunfälle betrachtet, bei denen es sich nicht um Alleinunfälle handelte, so ergibt sich eine prozentuale Verteilung im Hinblick auf den technischen Unfallverursacher, wie diese im nachfolgenden Diagramm wiedergegeben ist.

\section{Unfallverursacher}

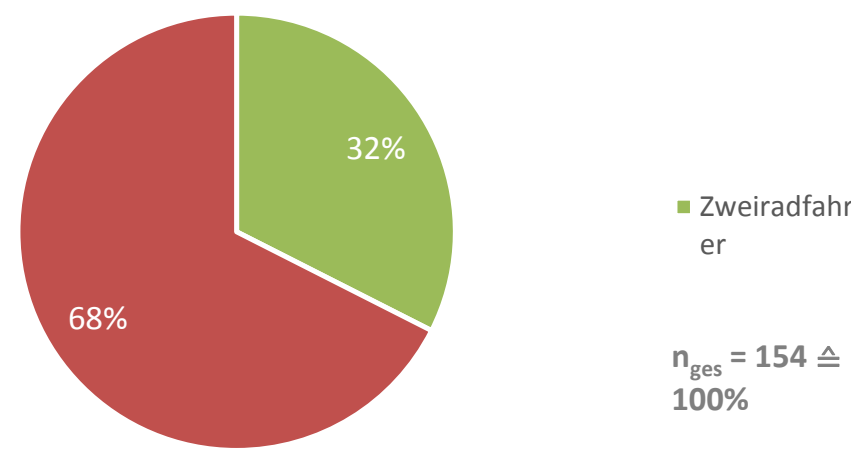

Diagramm 11:

Prozentuale Verteilung der untersuchten Verkehrsunfälle nach Unfallverursacher

\section{Unfallart}

Nach den Definitionen des statistischen Bundesamtes wird zwischen folgenden Unfallarten [1] unterschieden:

- Unfallart 1: Zusammenstoß mit einem anderen Fahrzeug, das anfährt, anhält oder im ruhenden Verkehr steht

- Unfallart 2: Zusammenstoß mit anderem Fahrzeug, das vorausfährt oder wartet

- Unfallart 3: Zusammenstoß mit anderem Fahrzeug, das seitlich in gleicher Richtung fährt

- Unfallart 4: Zusammenstoß mit anderem Fahrzeug, das entgegenkommt

- Unfallart 5: Zusammenstoß mit anderem Fahrzeug, das abbiegt, einbiegt oder kreuzt

- Unfallart 6: Zusammenstoß zwischen Fahrzeug und Fußgänger

- Unfallart 7: Aufprall auf ein Hindernis auf der Fahrbahn

- Unfallart 8: Abkommen von der Fahrbahn nach rechts

- Unfallart 9: Abkommen von der Fahrbahn nach links

- Unfallart 10: Unfall anderer Art 
Werden die im Erhebungszeitraum aufgenommenen Verkehrsunfälle entsprechend kategorisiert, so ergibt sich die im Diagramm 12 aufgezeigte prozentuale Verteilung.

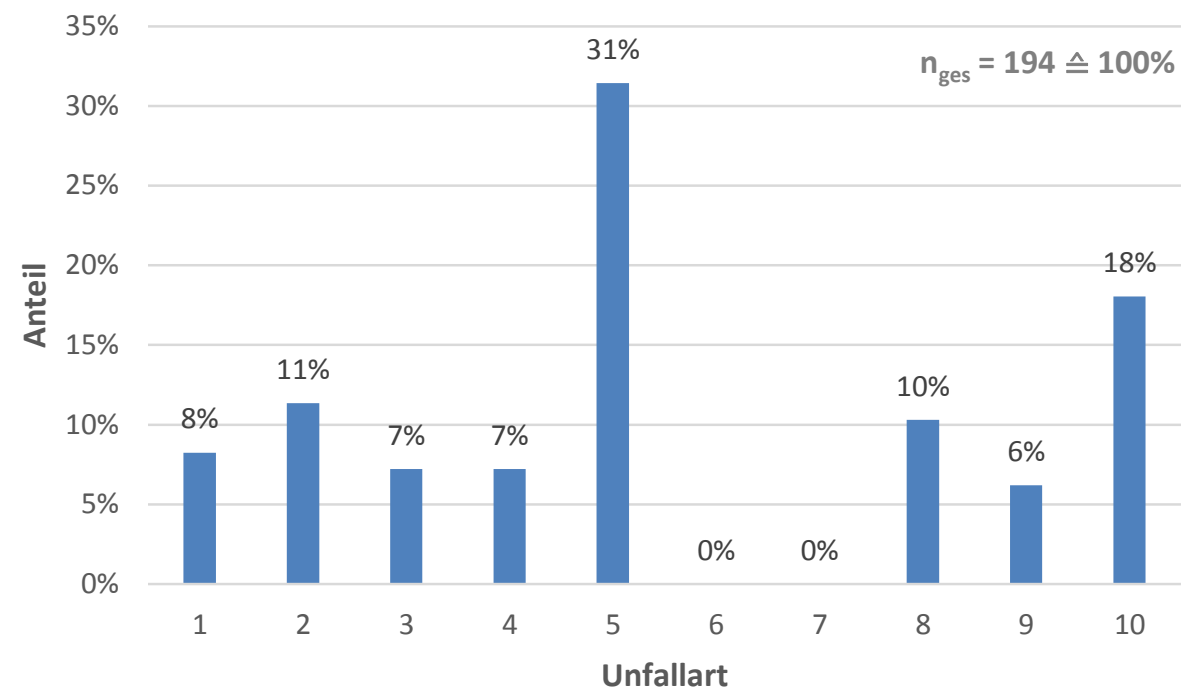

Diagramm 12:

Prozentuale Verteilung der untersuchten Verkehrsunfälle nach Unfallart

Mit $31 \%$ konnten am häufigsten Verkehrsunfälle festgestellt werden, bei denen es zum Zusammenstoß mit einem anderen Fahrzeug kam, das abbog, einbog oder aber kreuzte (Unfallart 5).

\section{Unfalltyp}

Werden die untersuchten Verkehrsunfälle nach dem Unfalltyp [1] unterschieden, so ergibt sich das nachfolgend dargestellte Verteilungsmuster. Der Unfalltyp beschreibt die Konfliktsituation, die zum Unfall führte.

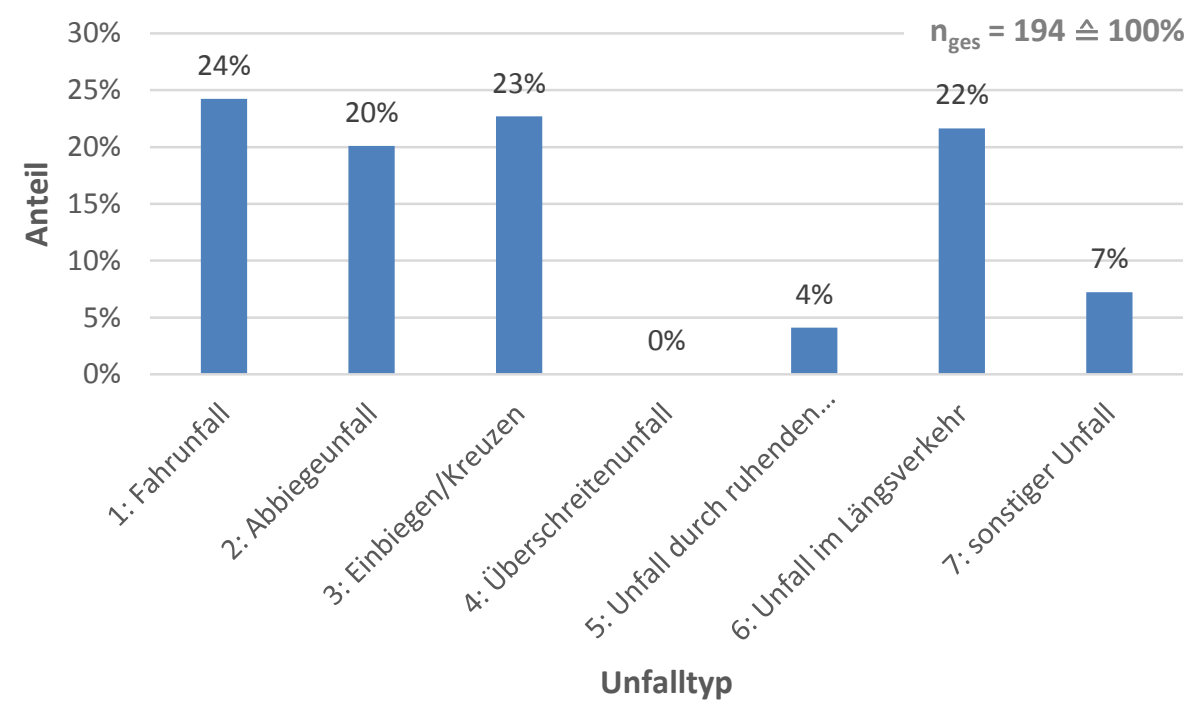

Diagramm 13:

Prozentuale Verteilung der untersuchten Verkehrsunfälle nach Unfalltyp

Als häufigster Unfalltyp zeigten sich Fahrunfälle, die auf ein Fehlverhalten des Zweiradfahrers ohne jegliche Fremdbeteiligung anderer Verkehrsteilnehmer zurückzuführen waren. Weiterhin kam 
es häufig zu sogenannten Einbiegen/Kreuzen-Unfällen, Unfällen im Längsverkehr aber auch Abbiegeunfällen mit einem jeweiligen Anteil von etwa $20 \%$.

\section{Unfallmechanismus}

Werden sämtliche untersuchten Verkehrsunfälle unter der Beteiligung von Krafträdern nach dem Unfallmechanismus des Zweirades unterschieden, so ergibt sich die nachfolgende Verteilung.

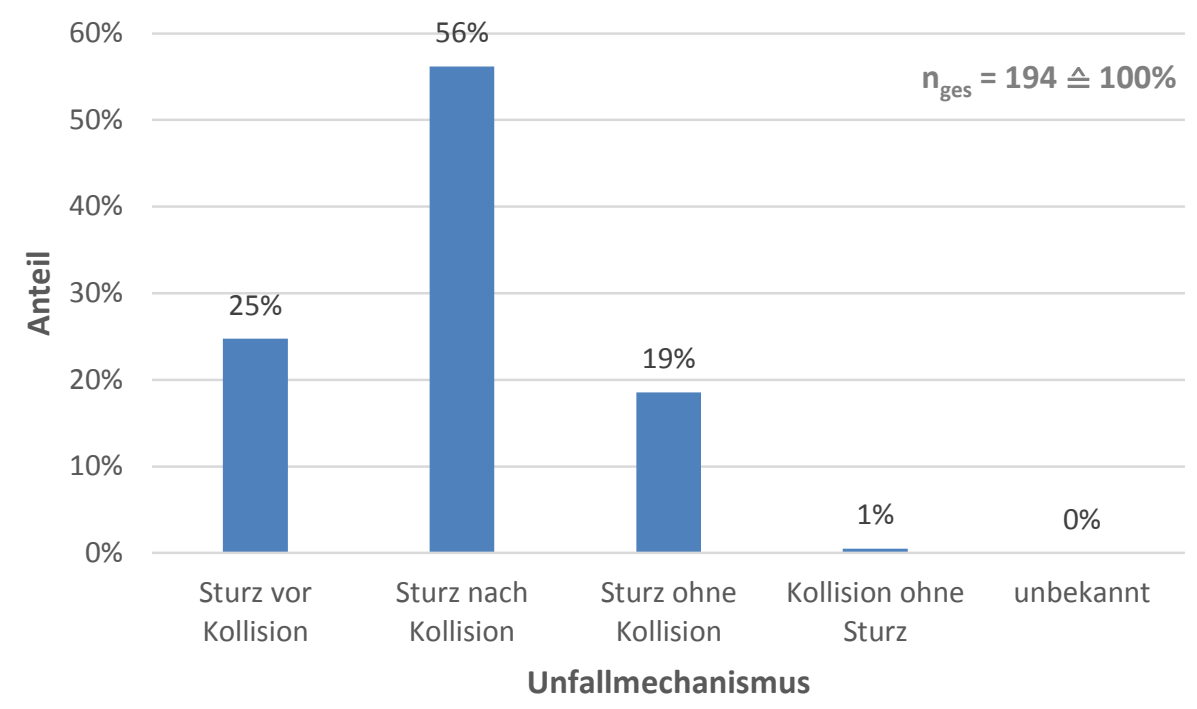

Diagramm 14:

Prozentuale Verteilung der untersuchten Verkehrsunfälle nach Unfallmechanismus des Zweirades

Im Zuge der Rekonstruktion der jeweiligen Unfallmechanismen wird deutlich, dass die Zweiradfahrer überwiegend (56\%) aus einer aufrechten Fahrposition heraus mit dem jeweiligen Kollisionspartner zusammengestoßen waren und es im Anschluss daran zu einem Sturz im Zuge der postkollisionären Auslaufphase kam.

\section{Ausrüstung der Krafträder mit ABS}

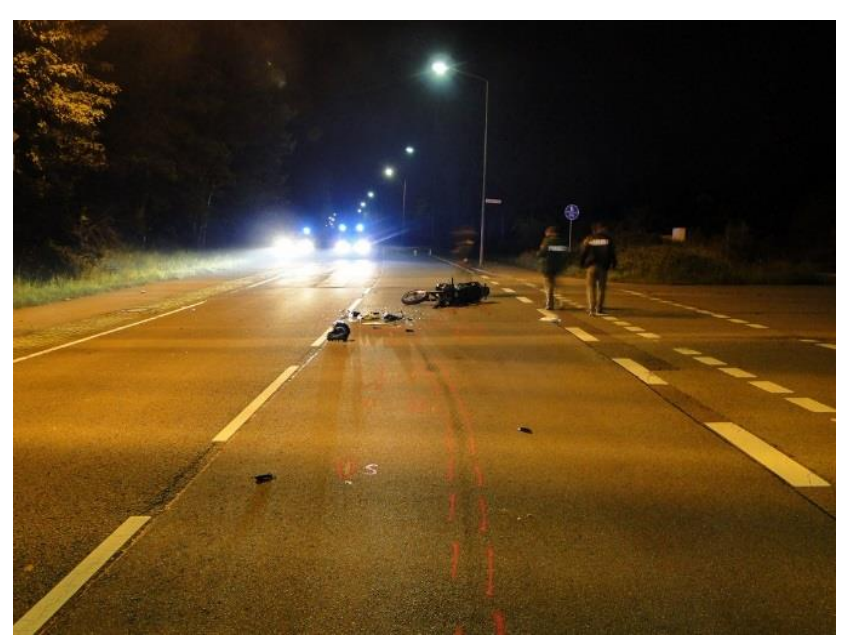

Wird das Diagramm 14 näher betrachtet, so zeigt sich weiterhin, dass es bei einem Viertel der Verkehrsunfälle im Erhebungszeitraum zu einem Sturz des Zweiradfahrers ohne Kollision im Vorfeld gekommen war. Ursächlich hierfür war in den meisten Fällen (ca. 83\%) die Einleitung einer starken Bremsverzögerung, die mit einem Blockieren eines Rades bzw. beider Räder einherging. Kam ein Kradfahrer zu Fall, ohne dass eine Kollision im Vorfeld bzw. im Nachhinein stattfand, ist es ebenfalls durchaus denkbar, dass zuvor eine starke Verzögerung

Abbildung 3:

Brems- bzw. Blockierspur Kraftrad mit einem Blockieren von zumindest einem Rad sturzursächlich war. Ein sogenanntes 
Antiblockiersystem, welches das Blockieren der Räder bei einer starken Abbremsung bzw. Vollverzögerung verhindert, könnte derartige Unfallgeschehen positiv beeinflussen bzw. teilweise möglicherweise sogar gänzlich vermeiden. Im Hinblick darauf wurde untersucht, ob die unfallbeteiligten Krafträder mit einem Antiblockiersystem ausgestattet waren. Die entsprechende Verteilung ist nachfolgend wiedergegeben. Weitergehende Auswertungen zum Unfallvermeidungspotenzial von ABS sind im Kapitel 12 wiedergegeben.

\section{Antiblockiersystem}

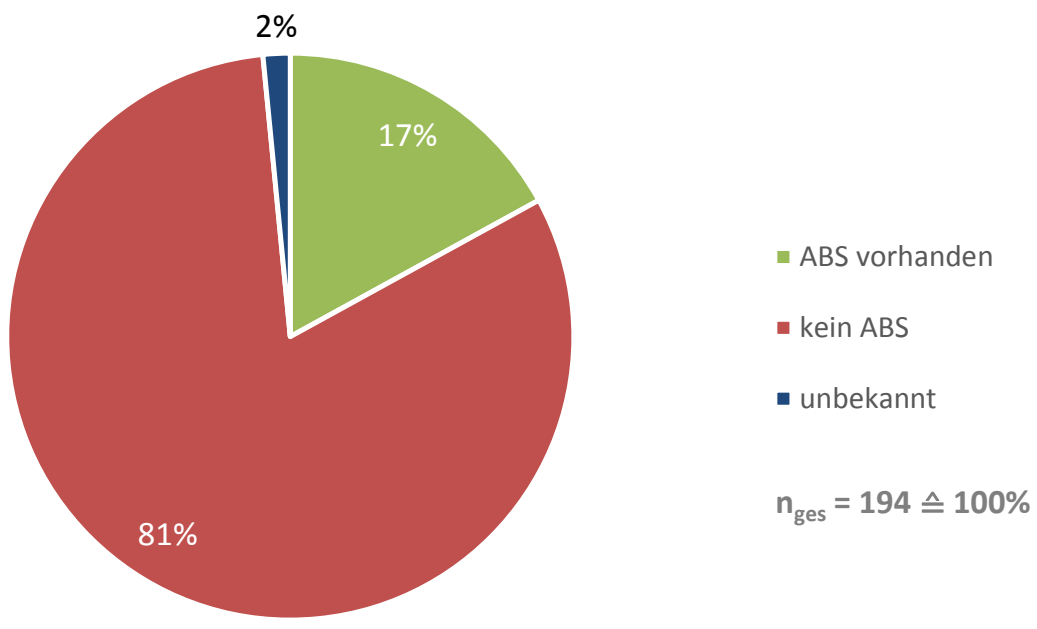

\section{Diagramm 15:}

Prozentuale Verteilung der untersuchten Krafträder im Hinblick auf Ausstattungsumfang Antiblockiersystem

Weniger als jedes fünfte unfallbeteiligte Kraftrad war mit ABS ausgestattet.

\section{Kollisionstyp}

Die Auswertung der Anstoßkonstellation der unfallbeteiligten Fahrzeuge nach ISO 13232 ist in der Abbildung 4 dargestellt. Hierbei wurden sämtliche untersuchten Verkehrsunfälle unter der Beteiligung von Krafträdern, bei denen es zu einer Kollision mit einem Pkw gekommen war, berücksichtigt $(n=127)$. 
Anstoßbereich am Pkw

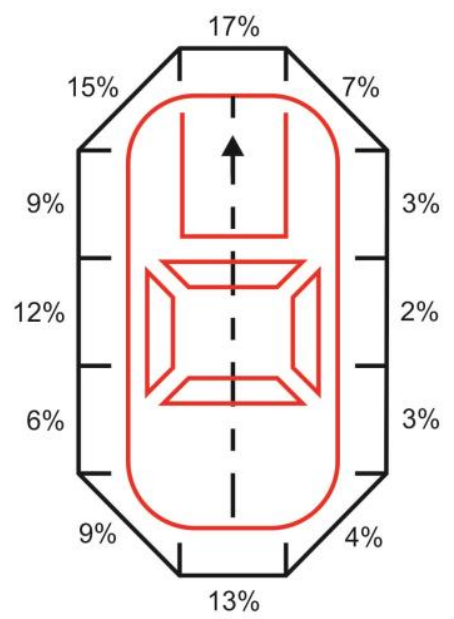

Anstoßbereich am Krad

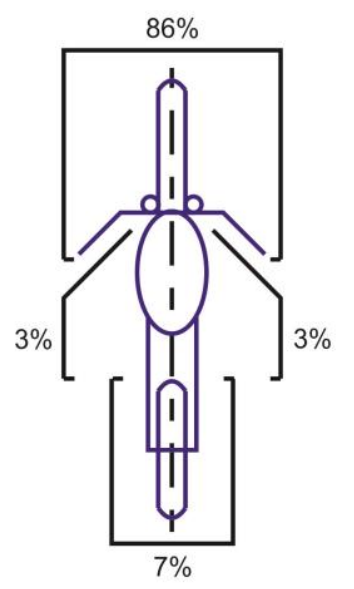

unbekannt: $1 \%$
Anstoßwinkel Pkw / Krad zueinander

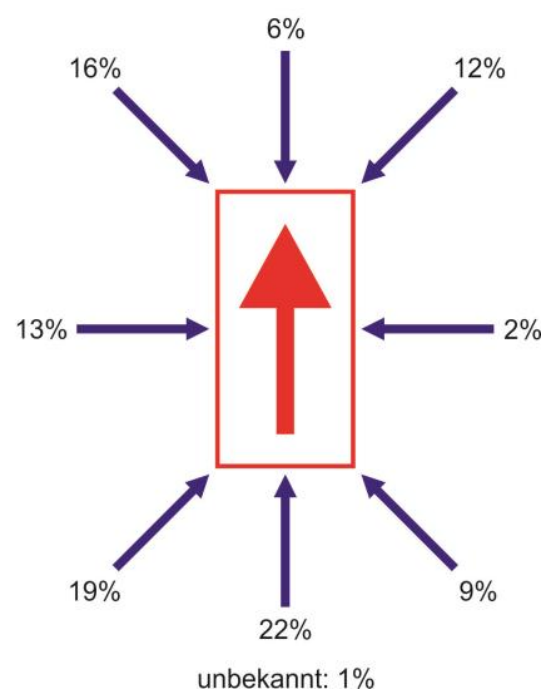

Abbildung 4:

Kollisionskonstellationen

Besonders häufig kam es zu einem Anstoß am unfallbeteiligten Pkw im Bereich der Fahrzeugfront. In ca. $86 \%$ der Fälle prallte das Kraftrad mit der Fahrzeugfront an seinen Kollisionsgegner. Recht häufig konnte festgestellt werden, dass die unfallbeteiligten Fahrzeuge in schräger Ausrichtung zueinander kollidiert waren. Eine weitere Häufung zeigt sich bei der Kollisionskonstellation in gleicher Fahrtrichtung bei nahezu längsachsenparalleler Ausrichtung.

Allerdings ist darauf hinzuweisen, dass die Auswertungen der Anstoßparameter jeweils Einzelbetrachtungen sind. Die Herleitung einer typischen Gesamtkollisionskonstellation bei Kombination der Einzelauswertungsergebnisse ist folglich nicht möglich.

\section{$8 \quad$ Auswertung unter medizinischen Gesichtspunkten}

Wie bereits dargelegt, wurde auch eine Vielzahl an medizinischen Parametern erhoben und ausgewertet. Hierbei wurden Feststellungen zu allgemeinen Informationen wie insbesondere Alter, Geschlecht, Größe sowie Gewicht der Zweiradfahrer getätigt. Aber auch die Fahrtüchtigkeit der Zweiradfahrer wurde überprüft.

Das tatsächliche Verletzungsbild wurde zudem im Hinblick auf die betroffene Körperregion sowie das Ausmaß erfasst. Auch wurde zwischen oberflächlichen (Hämatome, Hauteinblutungen, Schürfungen, Riss- oder Schnittwunden) sowie tieferliegenden Verletzungsmustern (Frakturen, Prellungen, Verletzungen innerer Organe) unterschieden. Der Bewusstseinszustand und die initial gemessenen Vitalparameter wurden ebenso erfasst.

Die Einstufung bzw. Kategorisierung der Einzelverletzungen nach Körperregionen erfolgte nach der Abbreviated Injury Scale (AIS 1990 Revision Update 98 [8]). Bei der AIS handelt es sich um einen Bewertungsmaßstab zur Letalität der erlittenen einzelnen Verletzungen. Die Verletzungsschwere wird hierbei in insgesamt sechs verschiedene Stufen (1-6) unterteilt. Darüber hinaus erfolgt eine Codierung nach der Art bzw. Ausprägung der Verletzung sowie der entsprechenden Lage. 
$\mathrm{Da}$ allerdings durch die AIS-Codierung lediglich Einzelverletzungen erfasst bzw. spezifiziert werden, ist zunächst keine Beurteilung der Gesamtverletzungsschwere einer Person möglich. Mit Hilfe der Maximum Abbreviated Injury Scale (MAIS) kann die gesamte Verletzungsschwere einer Person beurteilt werden. In Abweichung zur allgemein üblichen Definition wird im Folgenden der MAIS darüber hinaus auch zur Beschreibung der schwersten Verletzungen einer Körperregion angewendet. Zur Bestimmung des entsprechenden MAIS-Wertes wird der Maximalwert der Einzelverletzungen zwischen 1 und 6 berücksichtigt.

Die Beurteilung des Verletzungsmusters wurde darüber hinaus unter Zuhilfenahme des Injury Severity Score (ISS) [12] durchgeführt. Der ISS-Wert ergibt sich aus der Summe der Quadrate der AIS-Werte der drei am schwersten verletzten Körperregionen. Es können sich Werte zwischen 0 und 75 ergeben. Ergeben sich ISS-Werte $\geq 16$, so spricht man von einem sogenannten Polytrauma. Im allgemeinen medizinischen Sprachgebrauch zählen polytraumatisierte Patienten zu den Schwerstverletzten, wobei mehrere gleichzeitig geschehene Verletzungen verschiedener Körperregionen vorliegen und mindestens eine Verletzung oder die Kombination mehrerer Verletzungen als lebensbedrohlich anzusehen sind.

Um möglichst vollständige Datensätze zu erhalten, war eine umfangreiche Nachbearbeitung notwendig, da es nur teilweise bereits am Unfallort möglich war, alle erforderlichen Parameter zu erheben. Diese bestand aus z. B. der persönlichen Kontaktaufnahme zu den betroffenen Zweiradfahrern und der Einsichtnahme der Krankenakte. Eine vollständige Datenerhebung unter medizinischen Gesichtspunkten war dennoch nicht in allen Fällen möglich. In diesem Zusammenhang ist darauf hinzuweisen, dass personenbezogene Daten anonymisiert erfasst wurden und weitergehende Informationen (z. B. Einsichtnahme in Patientenakten) nur dann erfasst wurden, wenn eine ausdrückliche Zustimmung durch die entsprechenden Motorradaufsassen gegeben wurde.

Im Zuge der Besichtigung der getragenen Bekleidungsgegenstände der Zweiradaufsassen wurde im Wesentlichen überprüft, ob spezielle Schutzkleidung getragen wurde und auch das möglicherweise vorhandene Beschädigungsbild an der Bekleidung dokumentiert.

\section{Altersverteilung der Unfallbeteiligten}

Bei den untersuchten Verkehrsunfällen wurden sowohl die beteiligten Zweiradfahrer als auch die Unfallgegner in Altersgruppen unterteilt. Die entsprechende prozentuale Verteilung ist im nachfolgend dargestellten Diagramm wiedergegeben. 


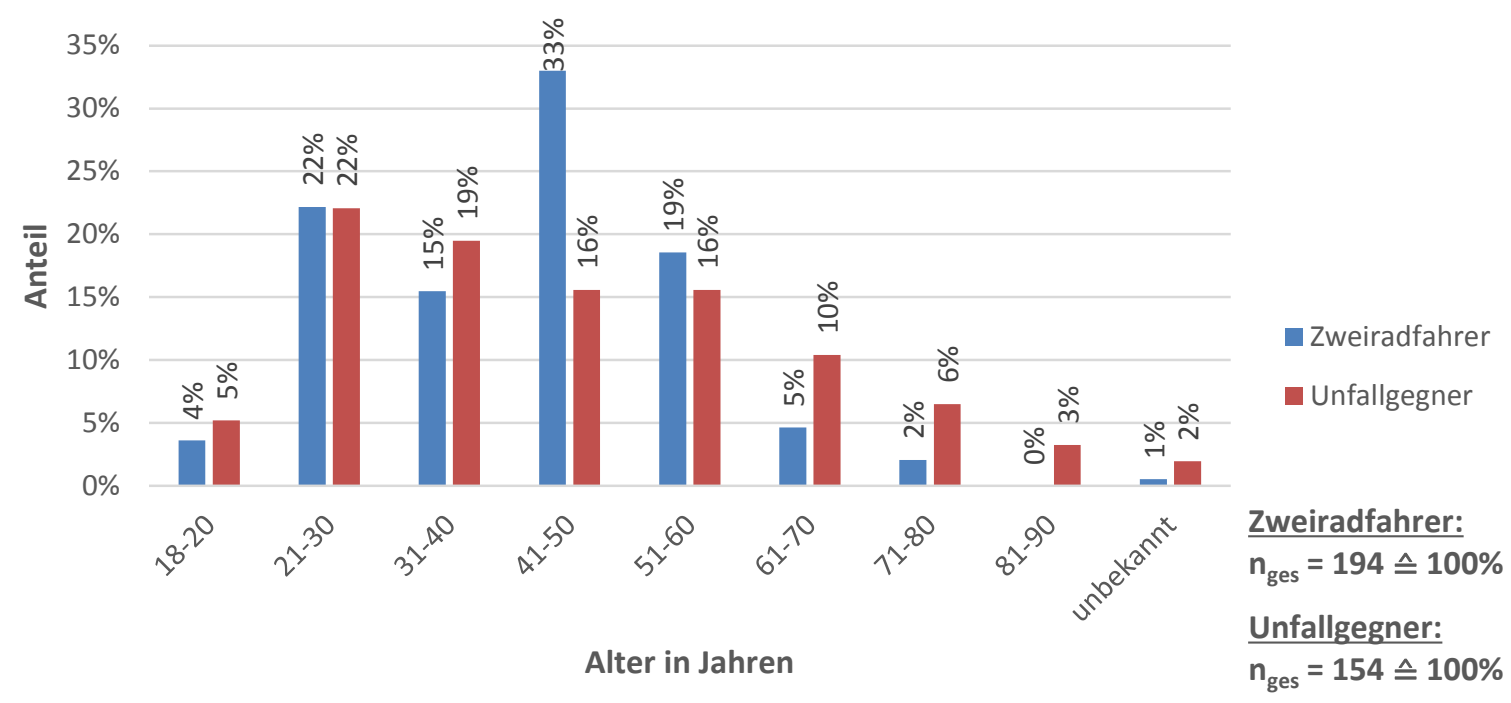

Diagramm 16:

Prozentuale Verteilung der untersuchten Verkehrsunfälle nach Alter der Unfallbeteiligten

Ca. ein Drittel der verunfallten Zweiradfahrer konnte der Gruppe der 41- bis 50-Jährigen zugeordnet werden. Darüber hinaus zeigte sich ein recht hoher Anteil von ca. $26 \%$ in der Altersgruppe der 18-bis 30-jährigen Zweiradfahrer.

Auch besonders häufig waren Unfallgegner im Alter zwischen 18 und 30 Jahren an den untersuchten Verkehrsunfällen beteiligt. Sie stellten einen Anteil von insgesamt 27\% dar.

\section{Fahrtüchtigkeit}

Bei den im Erhebungszeitraum untersuchten Verkehrsunfällen konnte in Einzelfällen eine Fahruntüchtigkeit der Zweiradfahrer nachvollzogen werden. Hierbei handelte es sich im Wesentlichen um eine Beeinträchtigung der Fahrtüchtigkeit durch Alkoholeinfluss, wie dies im nachfolgend dargestellten Diagramm aufgezeigt ist.

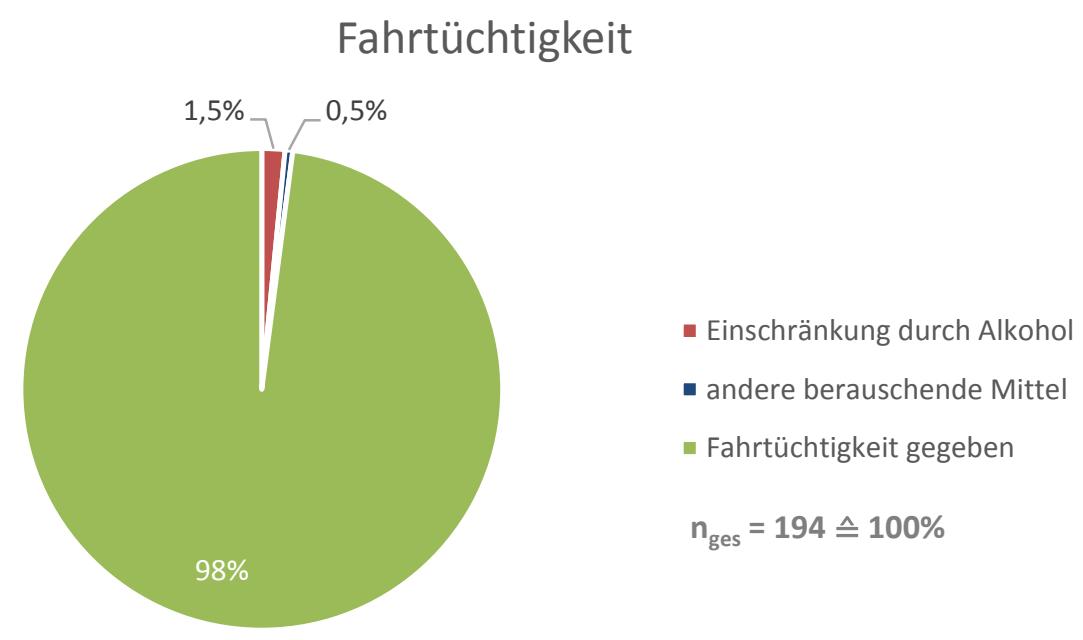

Diagramm 17:

Prozentuale Verteilung der Fahrtüchtigkeit der Zweiradfahrer 
In insgesamt drei Fällen konnte somit nachvollzogen werden, dass die Fahrtüchtigkeit der Zweiradfahrer durch Alkoholeinfluss deutlich beeinträchtigt war. Darüber hinaus konnte in einem Fall der Einfluss von illegalen Drogen nachgewiesen werden.

\section{Verletzungsschwere nach polizeilicher Erhebung}

Die prozentuale Verteilung der von den Unfallforschungsteams untersuchten Verkehrsunfälle ist nachfolgend nach dem Verletzungsschweregrad der Zweiradfahrer dargestellt.

Die Verletzungsschwere ist wie nachfolgend wiedergegeben definiert:

- Getötete: Personen, die im Zeitraum von maximal 30 Tagen unfallbedingt sterben

- Schwerverletzte: Personen, die zur Behandlung der unfallbedingten Verletzungen stationär (mindestens 24 Stunden) in einem Krankenhaus aufgenommen werden

- Leichtverletzte: alle weiteren Verletzungsbilder

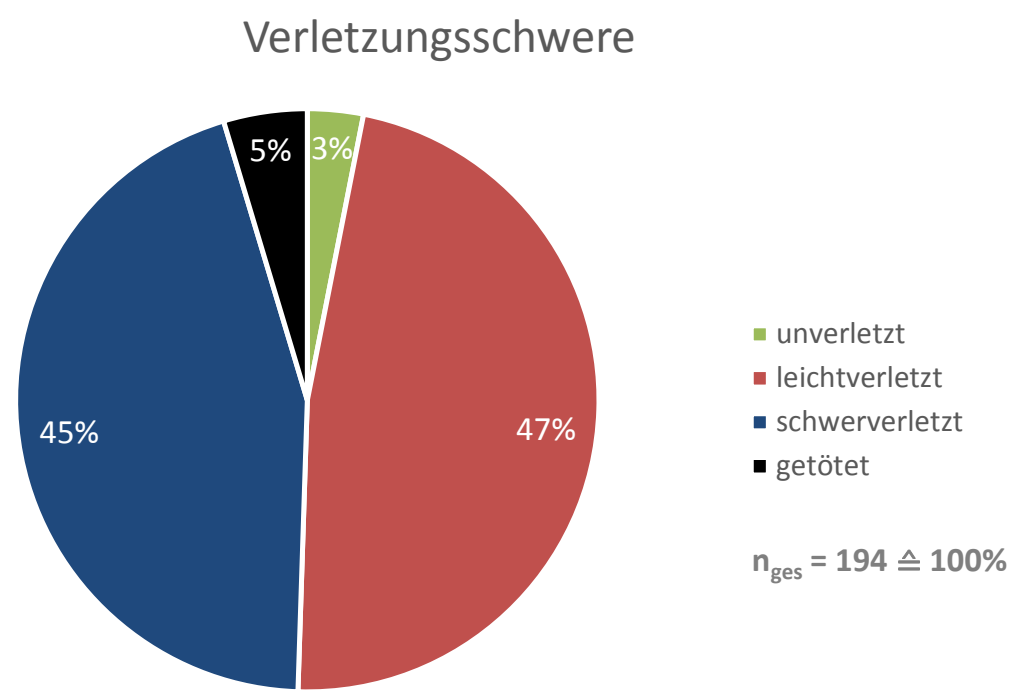

Diagramm 18:

Prozentuale Verteilung der untersuchten Verkehrsunfälle nach Verletzungsschwere der Zweiradfahrer

Nahezu jeder zweite Zweiradfahrer erlitt entsprechend der polizeilichen Definition schwere Verletzungen.

\section{Verletzungsschwere nach Körperregion}

Werden die analysierten Verkehrsunfälle im Hinblick auf die Verletzungsschwere der Motorradfahrer nach der jeweiligen Körperregion (nach MAIS-Werten) ausgewertet, so ergibt sich eine prozentuale Verteilung, wie diese im nachfolgenden Diagramm dargestellt ist. 


\section{Verletzungsschwere nach Körperregion}

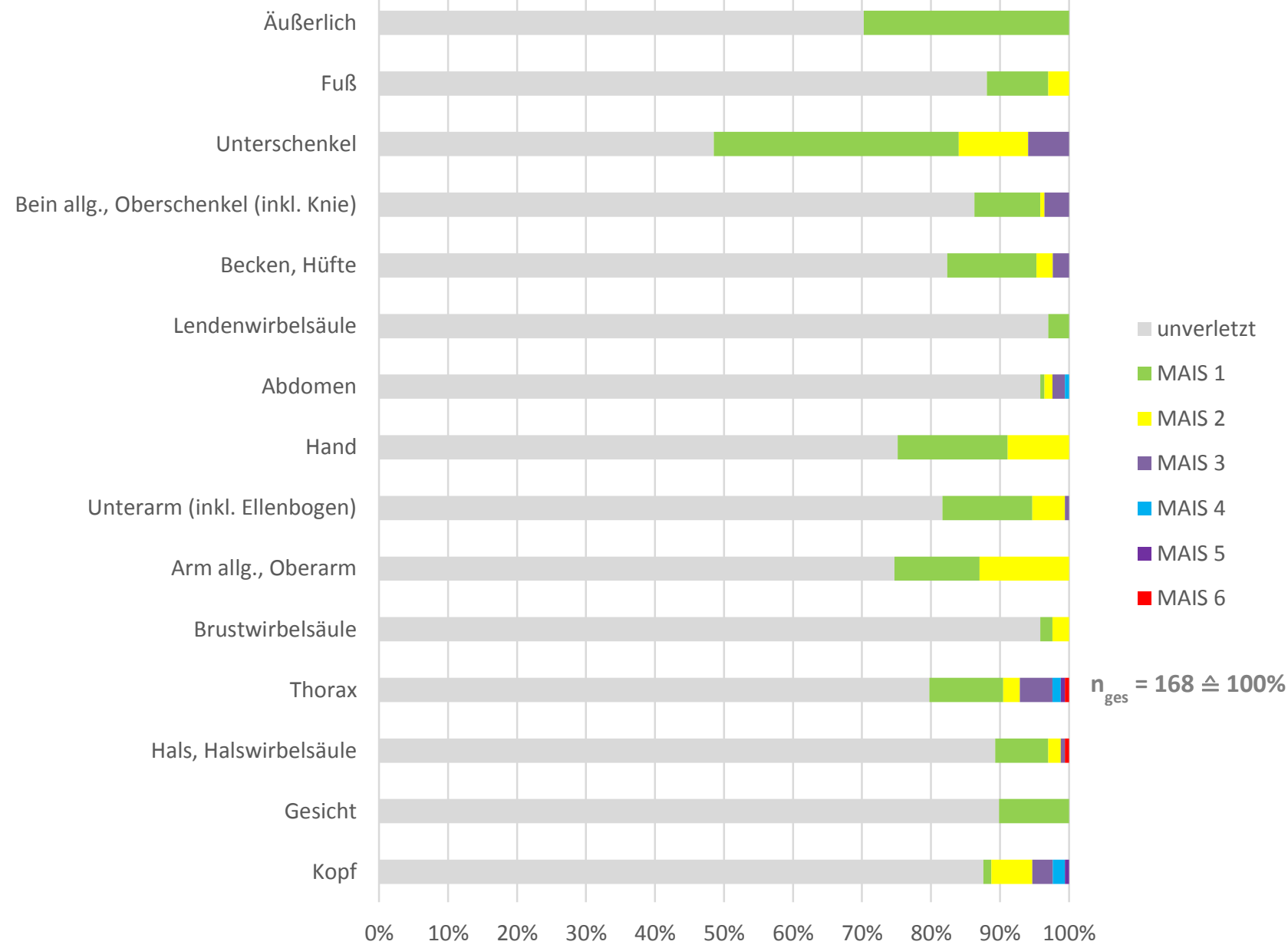

Diagramm 19:

Verletzungsschwere nach Körperregion

Das Diagramm verdeutlicht, dass es zu besonders schweren Verletzungen (MAIS 4-6) ausschließlich in den Bereichen Abdomen, Thorax und Kopf gekommen war. Darüber hinaus zeigten sich am häufigsten Verletzungen am Unterschenkel sowie äußerliche Verletzungen.

Werden die einzelnen Körperregionen weiter zusammengefasst und entsprechend gruppiert, so wird deutlich, dass es grundsätzlich neben den äußerlichen Verletzungen (beispielsweise Schürfungen, Prellungen) besonders häufig zu Verletzungen der oberen und unteren Extremitäten gekommen war. Für weitere Einzelheiten wird auf die nachfolgende Tabelle verwiesen.

Tabelle 5:

Verletzungsschwere nach zusammengefassten Körperregionen

\begin{tabular}{|l|c|c|c|c|c|c|c|}
\hline & \multicolumn{7}{|c|}{ MAIS-Wert } \\
\hline & unverletzt & $\mathbf{1}$ & $\mathbf{2}$ & $\mathbf{3}$ & $\mathbf{4}$ & $\mathbf{5}$ & $\mathbf{6}$ \\
\hline Kopf & $88,9 \%$ & $6,3 \%$ & $2,6 \%$ & $1,2 \%$ & $0,6 \%$ & $0,2 \%$ & $0,2 \%$ \\
\hline Oberkörper & $87,8 \%$ & $6,3 \%$ & $2,4 \%$ & $2,4 \%$ & $0,6 \%$ & $0,3 \%$ & $0,3 \%$ \\
\hline Obere Extremitäten & $77,2 \%$ & $13,8 \%$ & $8,9 \%$ & $0,2 \%$ & $0,0 \%$ & & \\
\hline Bauch & $96,4 \%$ & $1,8 \%$ & $0,6 \%$ & $0,9 \%$ & $0,3 \%$ & $0,0 \%$ & $0,0 \%$ \\
\hline Untere Extremitäten & $76,3 \%$ & $16,7 \%$ & $4,0 \%$ & $3,0 \%$ & $0,0 \%$ & $0,0 \%$ & $0,0 \%$ \\
\hline Äußerlich & $70,2 \%$ & $29,8 \%$ & & $0,0 \%$ & $0,0 \%$ & $0,0 \%$ & $0,0 \%$ \\
\hline
\end{tabular}




\section{Bekleidung}

Sofern es möglich war, wurde die Bekleidung der Zweiradaufsassen durch die Unfallforschungsteams noch im Bereich der Unfallörtlichkeit in Augenschein genommen. Hierbei wurde zunächst überprüft, ob es sich hierbei um spezielle Motorradschutzbekleidung handelte und zudem wurden eventuell vorliegende Beschädigungen erfasst.

Nicht in allen Fällen war eine vollständige Analyse der getragenen Bekleidungsgegenstände am Unfallort möglich, da die Zweiradaufsassen teilweise bei Ankunft der Unfallforschungsteams bereits im Zuge der medizinischen Versorgungsmaßnahmen abtransportiert worden waren. Allerdings konnten teilweise auch Informationen zur getragenen Bekleidung nachträglich erhoben und entsprechend ausgewertet werden. Die nachfolgend dargestellten Auswertungsergebnisse beziehen sich somit auf eine Teilmenge aller aufgenommenen Verkehrsunfälle.

\section{Schutzhelm}

Die prozentuale Verteilung der durch die Zweiradaufsassen getragenen Schutzhelme ist im Diagramm 20 nach dem entsprechenden Helmtyp dargestellt. Die unfallbeteiligten Zweiradaufsassen trugen überwiegend (83\%) einen Integralschutzhelm. Es konnte festgestellt werden, dass alle Motorradaufsassen einen Schutzhelm zum Unfallzeitpunkt getragen hatten.

\section{Schutzhelm}

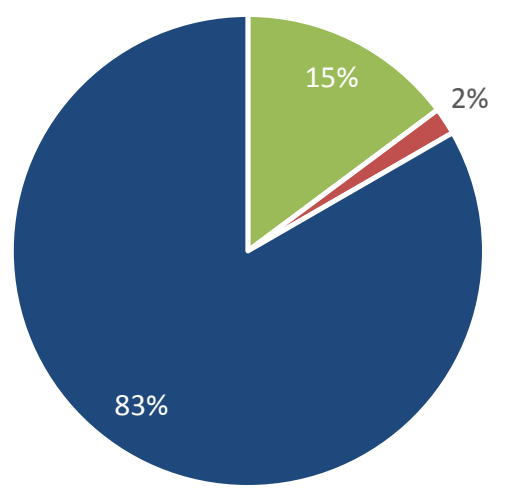

- Jethelm

- Endurohelm

- Integralhelm

$\mathrm{n}_{\mathrm{ges}}=162 \bumpeq 100 \%$

\section{Diagramm 20:}

Prozentuale Verteilung getragener Schutzhelme nach Typ

\section{Schutzjacke}

Die prozentuale Verteilung der durch die Zweiradaufsassen getragenen Oberkörperbekleidung nach Ausführung ist im nachfolgenden Diagramm wiedergegeben. 


\section{Schutzjacke}

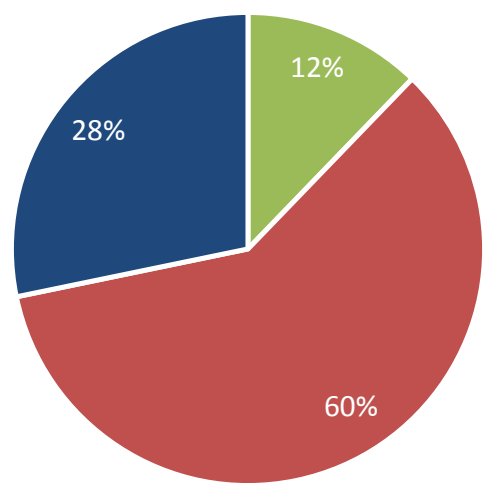

- keine Schutzjacke

- Schutzjacke Goretex

- Schutzjacke Leder

$\mathrm{n}_{\mathrm{ges}}=131 \triangleq 100 \%$

Diagramm 21:

Prozentuale Verteilung getragener Oberbekleidung nach Ausführung

\section{Schutzhose}

Im Diagramm 22 ist die prozentuale Verteilung der durch die Zweiradaufsassen getragenen Hosen im Hinblick auf die jeweilige Ausführung aufgezeigt.

\section{Schutzhose}

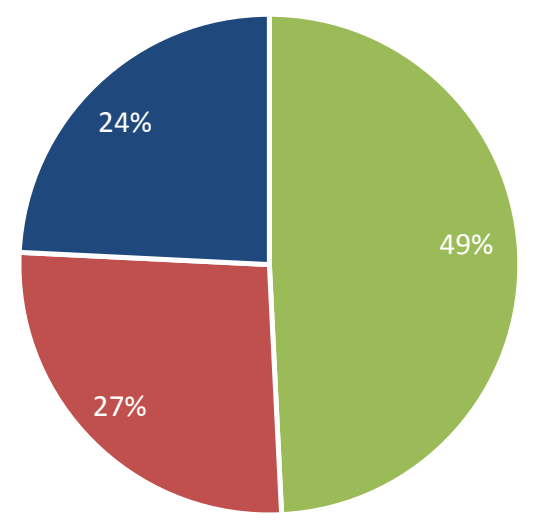

- keine Schutzhose

- Schutzhose Goretex

- Schutzhose Leder

$$
\mathrm{n}_{\mathrm{ges}}=128 \bumpeq 100 \%
$$

\section{Diagramm 22:}

Prozentuale Verteilung getragener Hosen nach Ausführung

\section{Schuhwerk}

Die prozentuale Verteilung des durch die Zweiradaufsassen getragenen Schuhwerks im Hinblick auf die jeweilige Art ist nachfolgend dargestellt. 


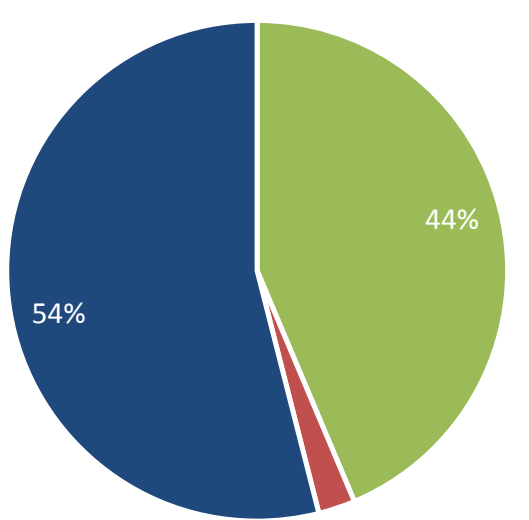

$2 \%$
- kein Sicherheitsschuhwerk

- Sicherheitsschuhwerk

- Motorradschutzstiefel

$\mathrm{n}_{\text {ges }}=126 \triangleq 100 \%$

\section{Diagramm 23:}

Prozentuale Verteilung des getragenen Schuhwerks nach Art

Die Auswertung der getragenen Bekleidungsstücke zeigt, dass durch die Kradfahrer in der Regel spezielle Motorradschutzkleidung getragen wurde. Allerdings zeigte sich im Hinblick auf das Schuhwerk, dass kaum mehr als jeder zweite Kradfahrer spezielle Motorradschutzstiefel getragen hatte. Jeder Kradfahrer war mit einem Schutzhelm ausgestattet, wobei es sich hier meistens (ca. $83 \%$ ) um Integralhelme gehandelt hatte.

\section{$9 \quad$ Unfallauswertungen nach Verursachungsmuster}

Zur weitergehenden Auswertung wurden alle im Erhebungszeitraum aufgenommenen Verkehrsunfälle $(n=194)$ nach dem Verursachungsmuster kategorisiert. Hierfür wurden die relevanten Feststellungen vor Ort zur Rekonstruktion herangezogen.

Die Einteilung erfolgte in die nachfolgend aufgeführten Kategorien:

- Alleinunfälle $(n=40)$

- Kradfahrer als Unfallverursacher $(n=50)$

- Unfallgegner als Unfallverursacher $(n=104)$

Die entsprechenden Einzelauswertungen der genannten Kategorien sind nachfolgend vergleichend wiedergegeben.

\section{Bauform des Kraftrades}

Im Diagramm 24 ist die prozentuale Verteilung der unfallbeteiligten Zweiräder nach der jeweiligen Bauform dargestellt. 


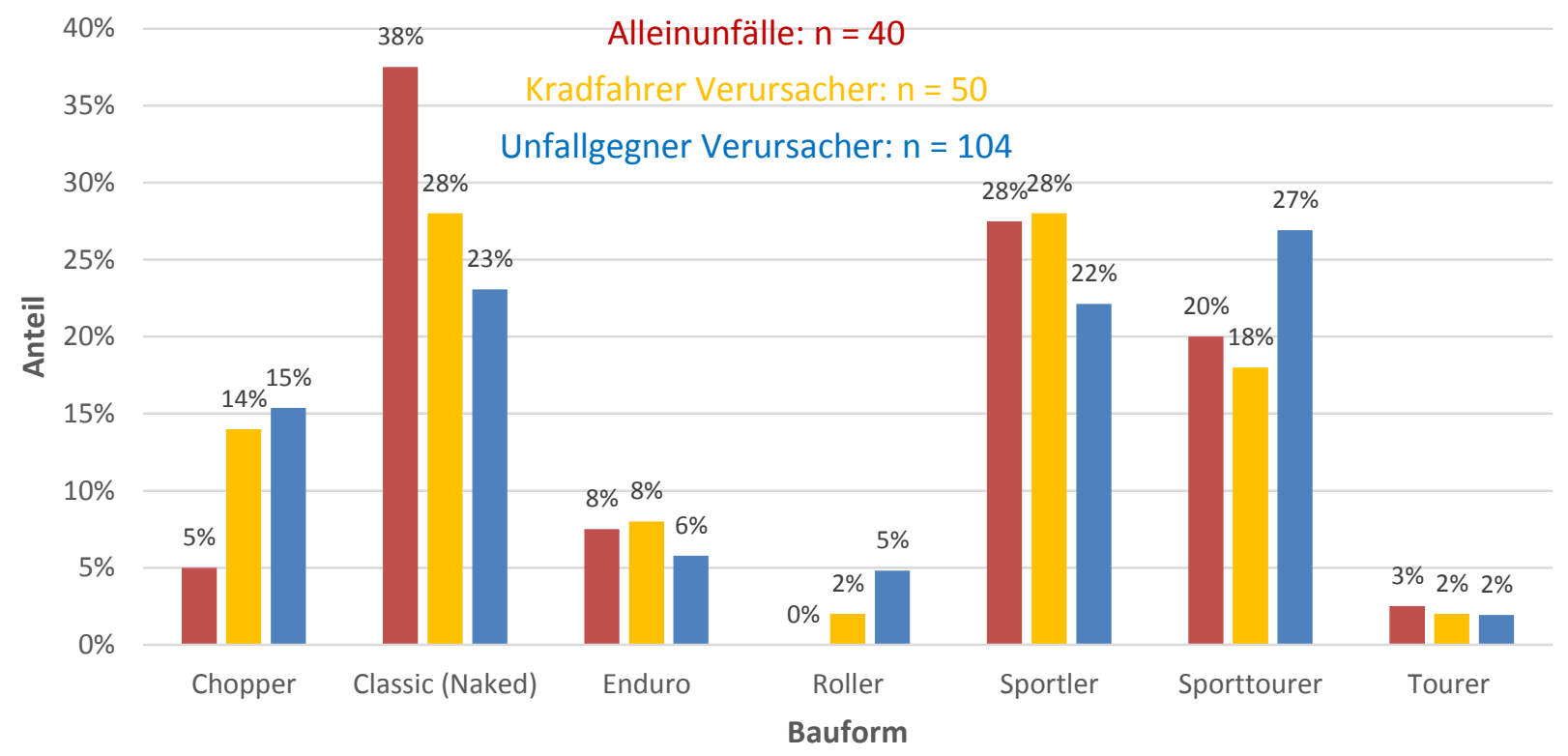

Diagramm 24:

Prozentuale Verteilung der untersuchten Verkehrsunfälle nach Krad-Bauform

Insbesondere bei Alleinunfällen sowie Unfällen mit mehreren Beteiligten, bei denen der Unfall durch den Kradfahrer verursacht wurde, zeigt sich eine hoher Anteil an sogenannten Naked-Bikes bzw. Sportlern.

\section{Wochentage}

Die Wochentagsverteilung aller von den Unfallforschungsteams untersuchten Verkehrsunfälle nach Unterteilung in die Unfallverursachungsmuster ist nachfolgend wiedergegeben.

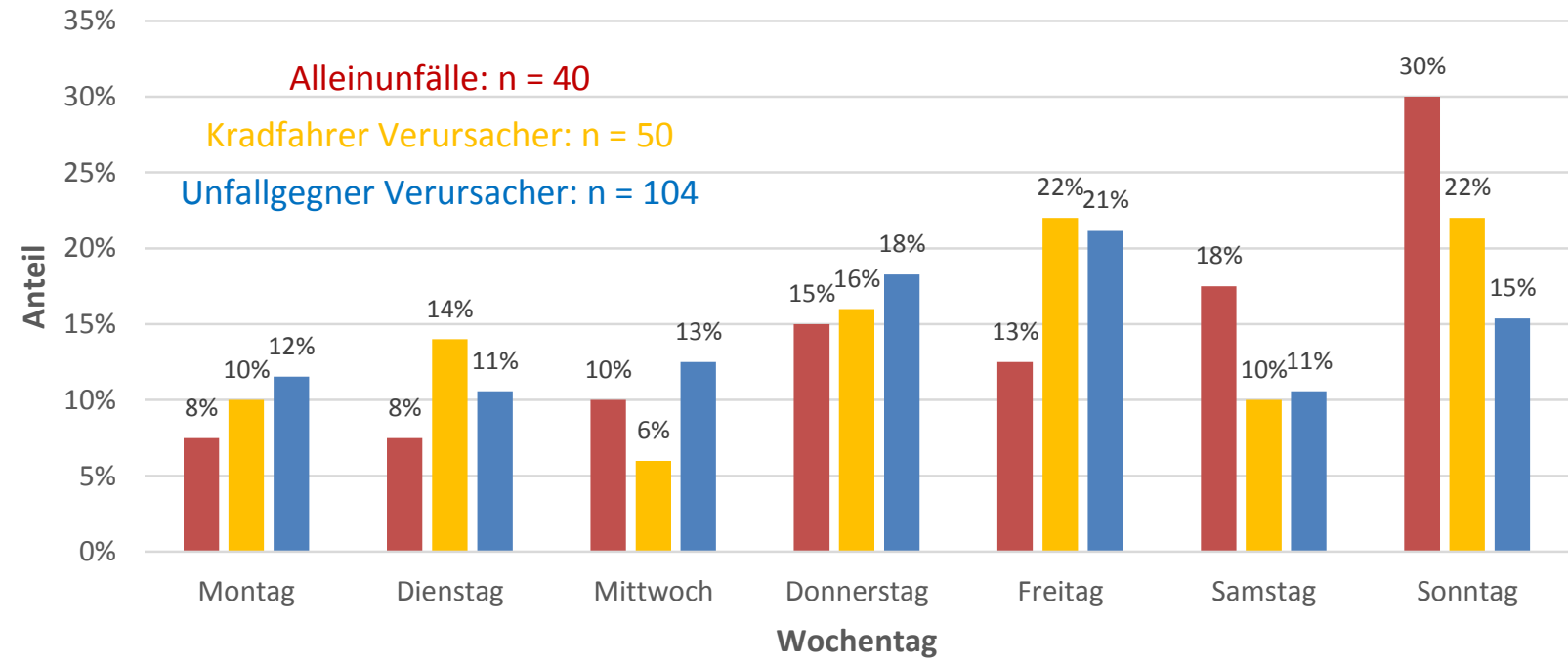

\section{Diagramm 25:}

Prozentuale Verteilung der untersuchten Verkehrsunfälle nach Wochentagen

Nahezu die Hälfte aller Alleinunfälle ereignete sich an den Tagen des Wochenendes (Samstag und Sonntag; 48\%). Verkehrsunfälle, die durch einen Unfallgegner verursacht wurden, ereigneten sich überwiegend innerhalb der Woche. 


\section{Uhrzeiten}

Die prozentuale Verteilung der untersuchten Verkehrsunfälle ist im nachfolgenden Diagramm nach Uhrzeiten dargestellt.

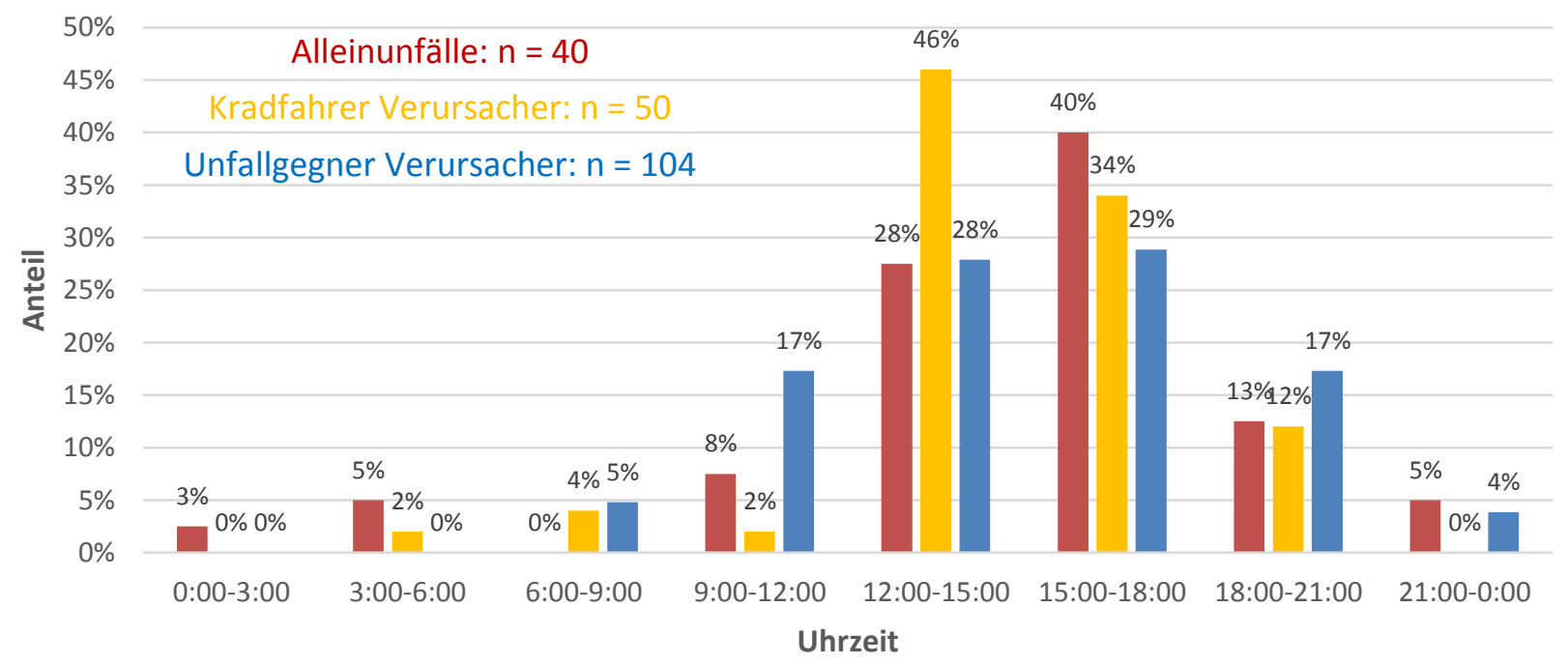

\section{Diagramm 26: \\ Prozentuale Verteilung der aufgenommenen Verkehrsunfälle nach Uhrzeiten}

Unabhängig vom Unfallverursachungsmuster ereignete sich der überwiegende Teil der analysierten Verkehrsunfälle im Zeitraum zwischen 12 und 18 Uhr.

\section{Ortslage}

Im Diagramm 27 ist die prozentuale Verteilung der untersuchten Verkehrsunfälle im Hinblick auf die Ortslage unter Kategorisierung nach dem Verursachungsmuster dargestellt.

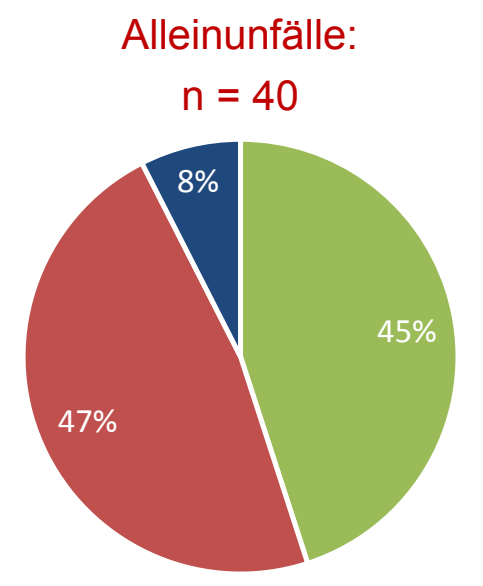

m innerorts a außerorts a Autobahn

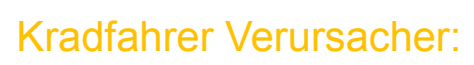

$$
n=50
$$

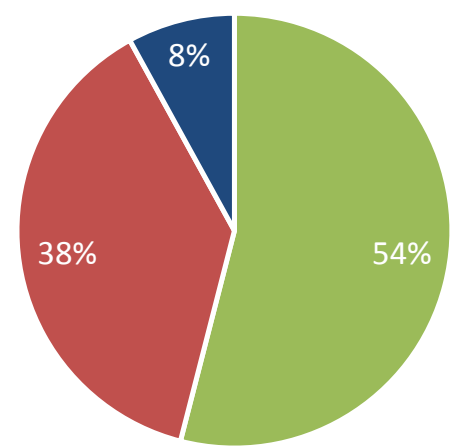

- innerorts a außerorts - Autobahn
Unfallgegner Verursacher:

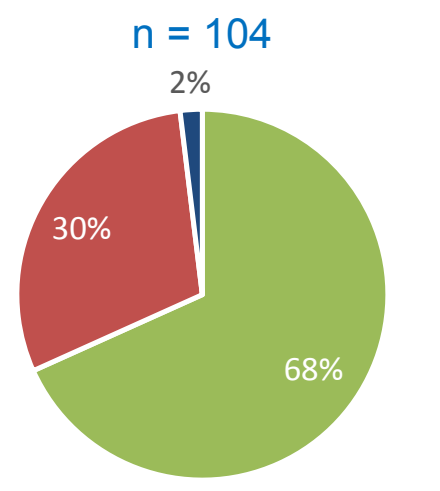

- innerorts a außerorts - Autobahn

Diagramm 27:

Prozentuale Verteilung der untersuchten Verkehrsunfälle nach Ortslage

Die Alleinunfälle ereigneten sich insbesondere außerorts, wohingegen es überwiegend innerorts zu Verkehrsunfällen kam, die durch einen Unfallgegner verursacht wurden. 


\section{Altersverteilung der Unfallbeteiligten}

Werden die im Erhebungszeitraum unfallbeteiligten Zweiradfahrer in Altersklassen unterteilt und wird weiterhin nach dem Verursachungsmuster kategorisiert, so ergibt sich die im nachfolgend dargestellten Diagramm aufgezeigte prozentuale Verteilung.

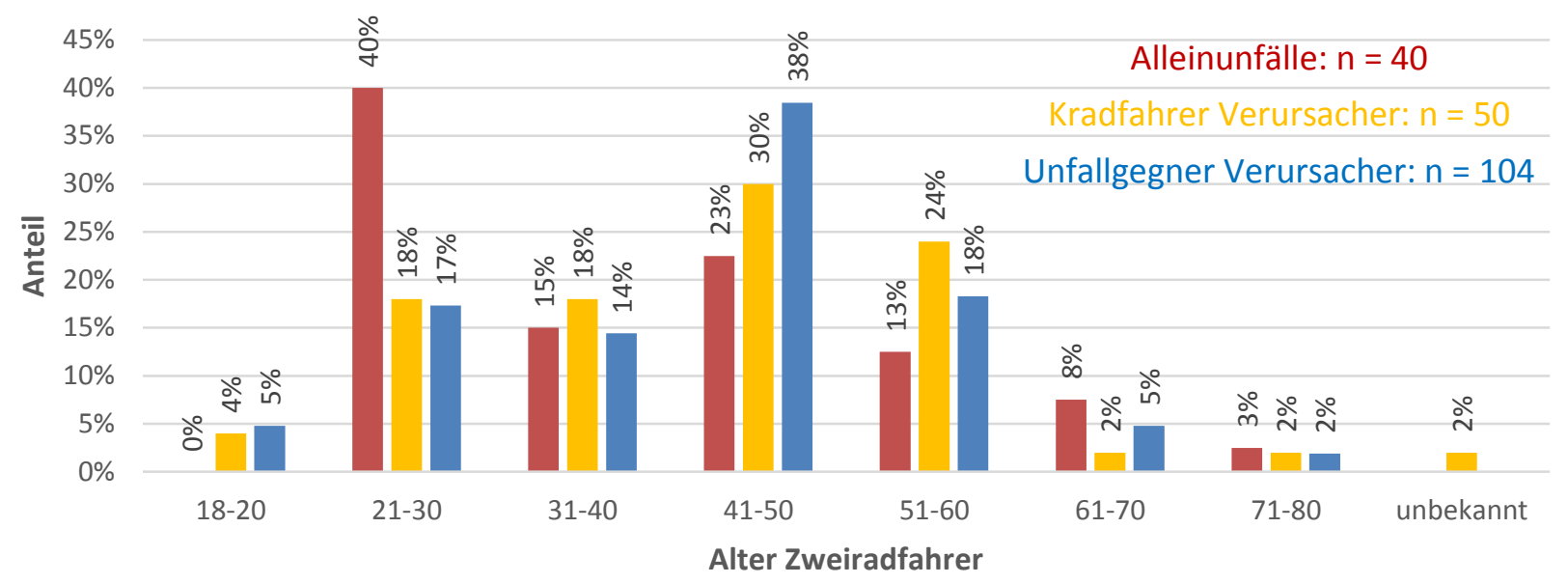

Diagramm 28:

Prozentuale Verteilung der untersuchten Verkehrsunfälle nach Alter der Zweiradfahrer

Besonders häufig kam es zu Alleinunfällen in der Gruppe der 21- bis 30-jährigen Zweiradfahrer. Bei mehreren Unfallbeteiligten kam es überwiegend zu Verkehrsunfällen, bei denen Zweiradfahrer im Altersbereich zwischen 41 und 60 Jahren beteiligt waren.

\section{Verletzungsschwere nach ISS}

Im Diagramm 29 ist die prozentuale Verteilung der untersuchten Verkehrsunfälle im Hinblick auf die Verletzungsschwere (ISS) unter Berücksichtigung des Verursachungsmusters dargestellt.
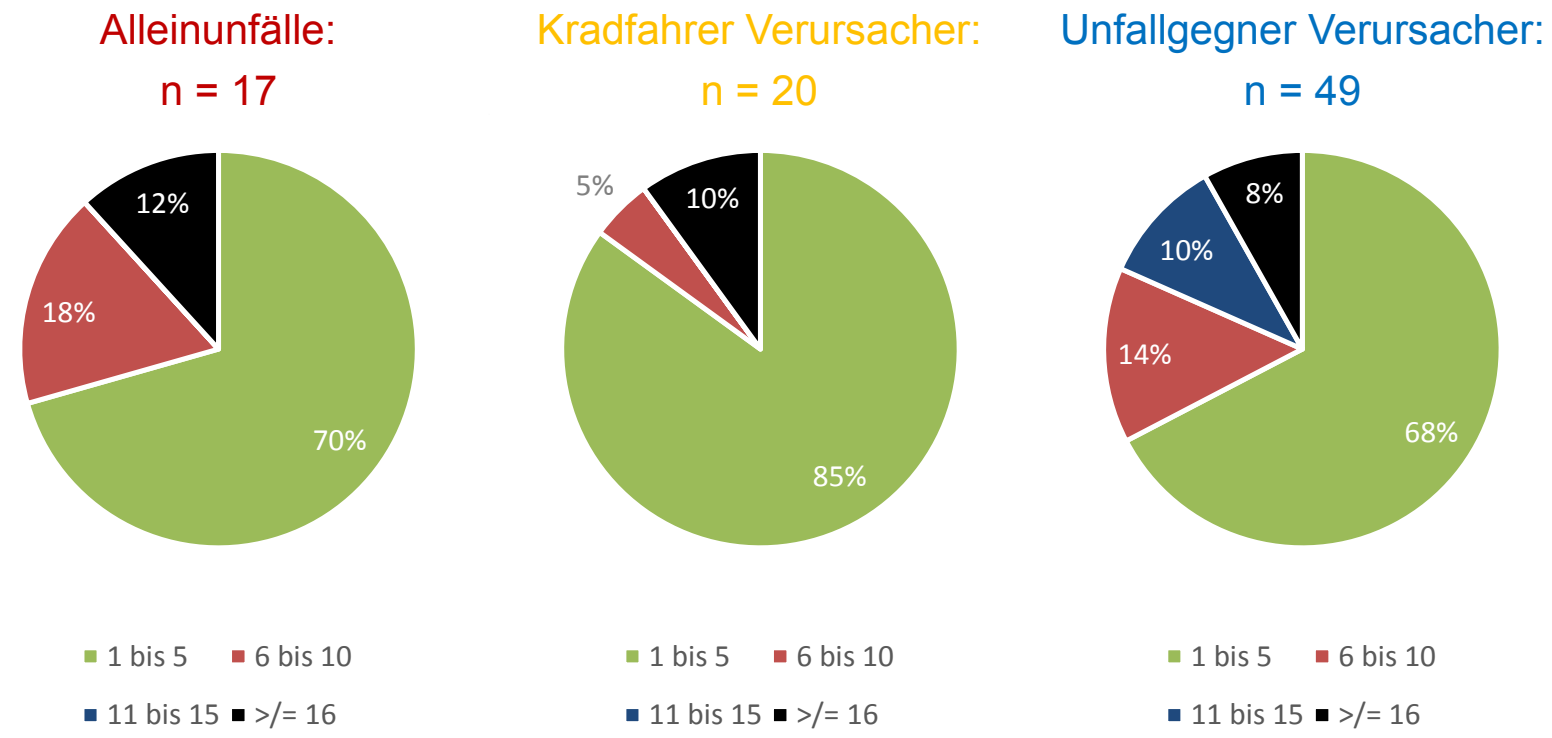

Diagramm 29:

Verletzungsschwere nach Injury Severity Score (ISS) 
Es zeigt sich, dass es überwiegend, unabhängig von dem Verursachungsmuster, zu ISS-Werten im Bereich von 1 bis 5 gekommen war. Schwerwiegende Verletzungen (ISS $\geq 16$ ) traten am häufigsten bei Alleinunfällen auf.

Werden alle schwerverletzten Zweiradaufsassen (nach polizeilicher Erhebung) berücksichtigt, so ergibt sich ein Anteil polytraumatisierter Zweiradaufsassen mit einem ISS $\geq 16$ von ca. 10\%. Bei derartigen Verletzungsbildern kann davon ausgegangen werden, dass mindestens eine Verletzung oder die Kombination mehrerer Verletzungen lebensbedrohlich sind.

\section{Verletzungsschwere nach MAIS}

Werden die analysierten Verkehrsunfälle nach dem Verursachungsmuster unterschieden, so ergibt sich die jeweilige prozentuale Verteilung der Verletzungsschwere nach MAIS, wie diese im nachfolgenden Diagramm dargestellt ist.

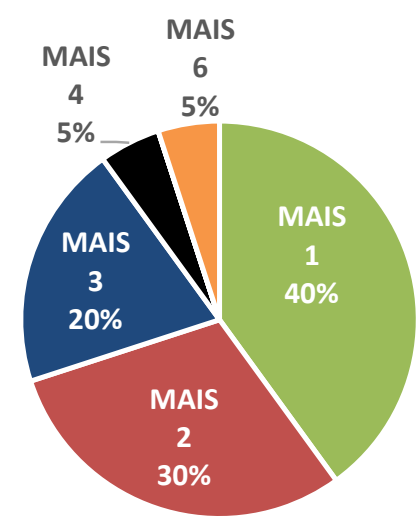

Alleinunfälle:

$$
n=20
$$

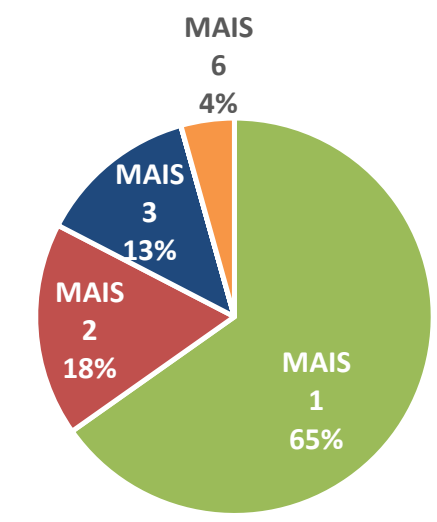

Kradfahrer Verursacher:

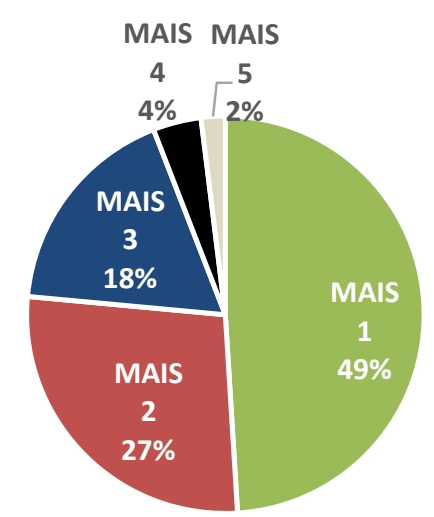

Unfallgegner Verursacher:

$$
n=51
$$

\section{Diagramm 30:}

Prozentuale Verteilung der Verletzungsschwere nach Maximum Abbreviated Injury Scale

$\mathrm{Zu}$ schwerwiegenden Verletzungen (MAIS 2+) kam es überwiegend bei Alleinunfällen (60\%). Zudem zeigt sich, dass es offensichtlich bei Verkehrsunfällen, die durch den Kradfahrer verursacht wurden (Alleinunfälle sowie Verkehrsunfälle mit mehreren Beteiligten), zu den schwersten Verletzungen (MAIS 6) gekommen war.

\section{Motorleistung der unfallbeteiligten Krafträder}

Die im Erhebungszeitraum unfallbeteiligten Krafträder wurden im Hinblick auf ihre Motorleistung untersucht und in Leistungsgruppen unterteilt. Hieraus ergibt sich die nachfolgend dargestellte prozentuale Verteilung unter Berücksichtigung des jeweiligen Verursachungsmusters. 
Alleinunfälle:

$\mathrm{n}=40$

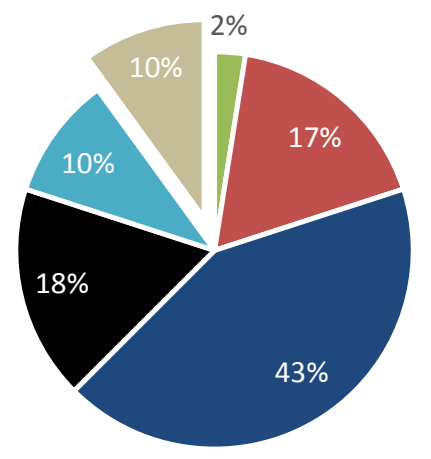

bis $25 \mathrm{~kW} \quad 26$ bis $50 \mathrm{~kW}$

- 51 bis 75 kW 76 bis 100 kW

äber $100 \mathrm{~kW}$ unbekannt
Kradfahrer Verursacher:

$n=50$

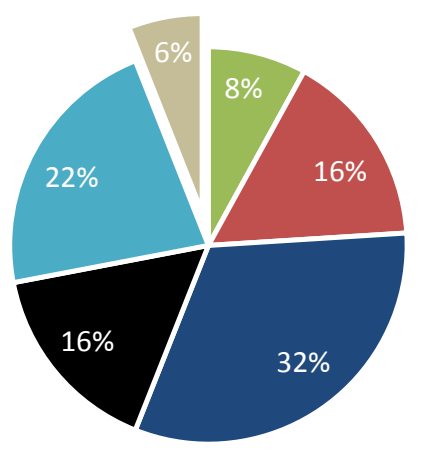

- bis $25 \mathrm{~kW} \quad 26$ bis $50 \mathrm{~kW}$

- 51 bis $75 \mathrm{~kW}$ - 76 bis $100 \mathrm{~kW}$

- über $100 \mathrm{~kW}$ - unbekannt
Unfallgegner Verursacher:

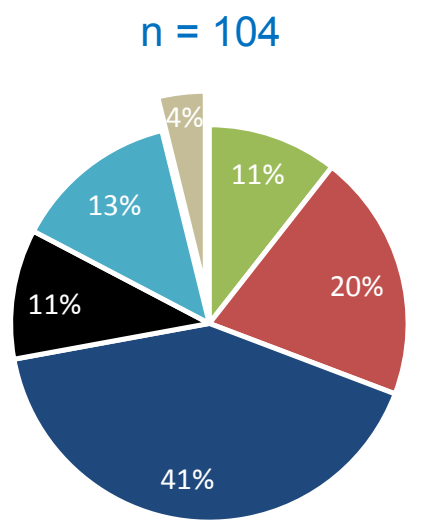

— bis 25 kW - 26 bis 50 kW
- 51 bis 75 kW - 76 bis 100 kW
über 100 kW unbekannt

Diagramm 31:

Prozentuale Verteilung der untersuchten Krafträder nach Motorleistung

Es konnte festgestellt werden, dass sich nur sehr wenige Alleinunfälle (2\%) unter der Beteiligung von recht leistungsschwachen ( $\leq 25 \mathrm{KW}$ bzW. $34 \mathrm{PS}$ ) Motorrädern ereignet hatten. Mehr als jedes fünfte Motorrad bei den Verkehrsunfällen mit mehreren Beteiligten, die durch den Kradfahrer verursacht wurden, wies eine Motorleistung über $100 \mathrm{KW}$ (entspricht $136 \mathrm{PS}$ ) auf.

\section{Unfallart}

Werden die im Erhebungszeitraum aufgenommenen Verkehrsunfälle nach dem Verursachungsmuster kategorisiert, so ergibt sich die nachfolgend dargestellte prozentuale Verteilung nach Unfallarten.

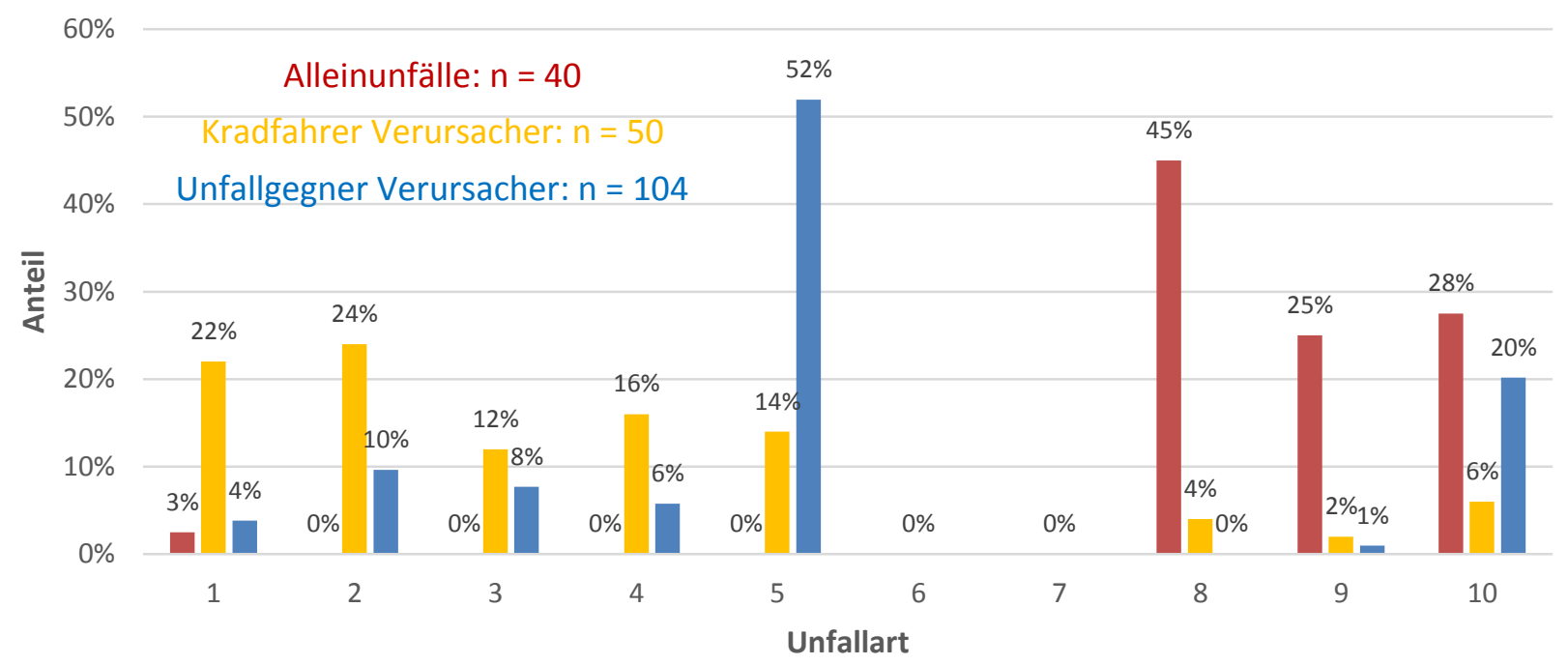

Diagramm 32:

Prozentuale Verteilung der untersuchten nach dem Verursachungsmuster kategorisierten Verkehrsunfälle nach Unfallart 
Ein Abkommen von der Fahrbahn (Unfallart 8 und 9) ist charakteristisch für den Alleinunfall. Wurden die Verkehrsunfälle durch den Kradfahrer verursacht, so zeigt sich, dass es hier überwiegend zu Auffahrkollisionen gekommen war (Unfallart 1 und 2). In mehr als der Hälfte der erhobenen Unfälle, die durch einen Unfallgegner verursacht wurden, kam es zu Zusammenstößen im abbiegenden, einbiegenden oder kreuzenden Verkehr (Unfallart 5) (52\%).

\section{Unfalltyp}

Werden die hier aufgenommenen Verkehrsunfälle nach dem Unfalltyp unterschieden, so zeigt sich, dass es sich bei den Alleinunfällen in der Regel um sogenannte Fahrunfälle handelte. Nahezu jeder zweite Unfall, der durch den Kradfahrer verursacht wurde, kann als Unfall im Längsverkehr eingegrenzt werden (48\%). Lag die Verursachung beim Unfallgegner, so zeigen sich die Schwerpunkte bei den analysierten Verkehrsunfällen bei den Einbiegen/Kreuzen- sowie Abbiegeunfällen.

Die entsprechenden Feststellungen sind im nachfolgenden Diagramm wiedergegeben.

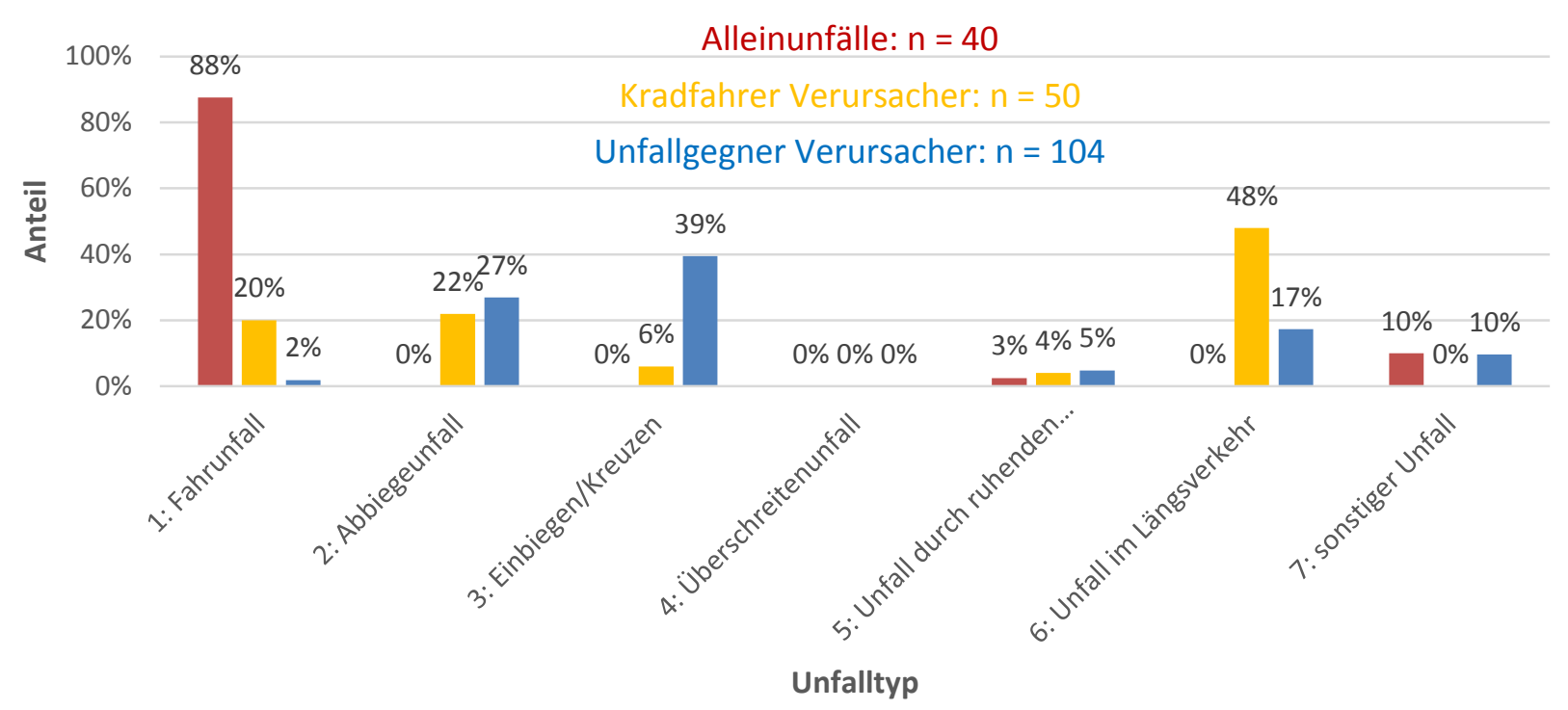

Diagramm 33:

Prozentuale Verteilung der untersuchten nach dem Verursachungsmuster kategorisierten Verkehrsunfälle nach Unfalltyp

\subsection{Vertiefende Analysen zum Alleinunfall}

Werden alle Alleinunfälle herangezogen und werden ferner lediglich die Unfallarten 8 (Abkommen von der Fahrbahn nach rechts) sowie 9 (Abkommen von der Fahrbahn nach links) berücksichtigt, so konnten weitere wesentliche Auswertungsergebnisse festgestellt werden. Beispielsweise ereignete sich nur etwa jeder dritte Verkehrsunfall innerhalb geschlossener Ortschaften. Weiterhin zeigte sich, dass es in $93 \%$ zu einem Abkommen von der Fahrbahn im Bereich von Kurvenverläufen (Linkskurven ca. 57\%, Rechtskurven ca. 36\%) kam. Nur in 18\% der Fälle konnte eine Reaktion vor dem Unfallereignis durch Einleitung einer Bremsverzögerung sicher nachvollzogen werden. 
Im Rahmen der Auswertung der Ursachen der aufgenommenen Krad-Alleinunfälle zeigte sich, dass deutlich mehr als die Hälfte aller Verkehrsunfälle durch nicht angepasste Geschwindigkeit und eine Überschreitung der örtlich zulässigen Geschwindigkeit verursacht wurden.

\section{Unfallursache}

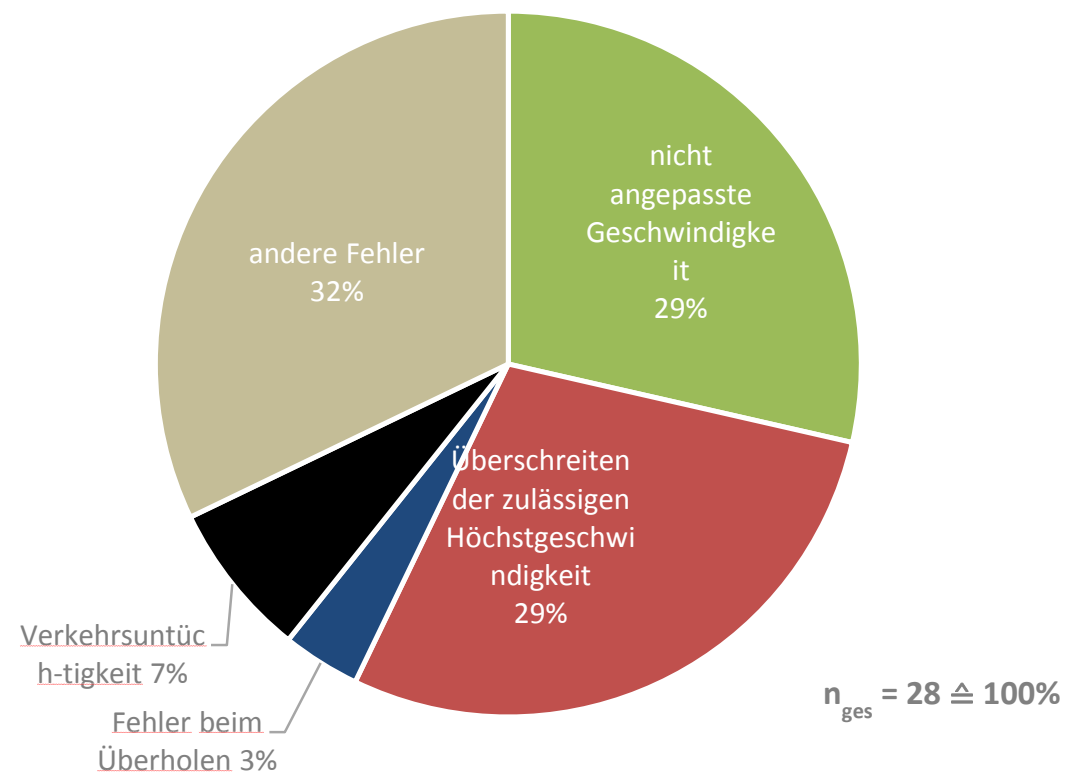

Diagramm 34:

Prozentuale Verteilung der Alleinunfälle nach Unfallursache

Werden die untersuchten Alleinunfälle nach dem Unfallablauf bzw. Unfallmechanismus des Zweirades unterschieden, so ergibt sich die im Diagramm 35 wiedergegebene Verteilung.

\section{Unfallmechanismus}

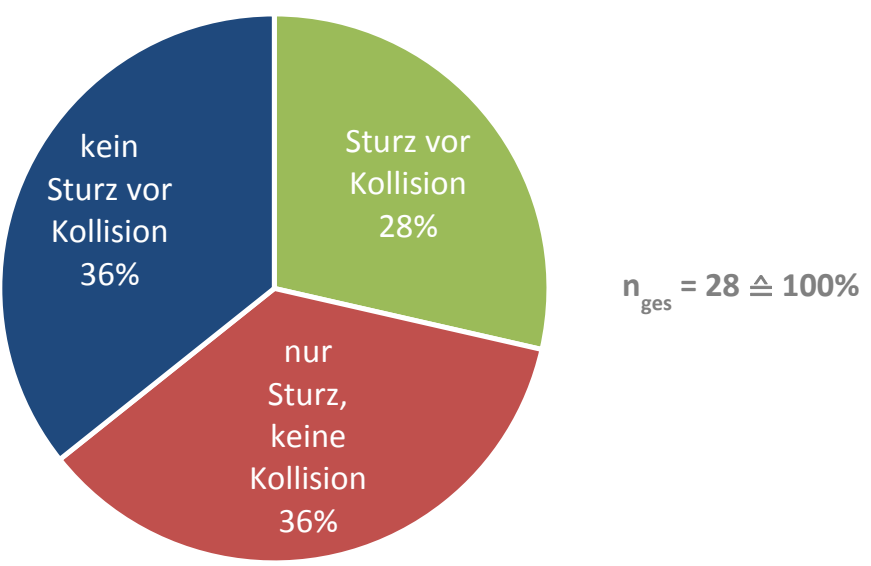

\section{Diagramm 35:}

Prozentuale Verteilung der Alleinunfälle nach Unfallmechanismus des Zweirades

Die Rekonstruktion des Unfalles verdeutlicht, dass es bei den hier analysierten Alleinunfällen am häufigsten zu Stürzen gekommen war, an die sich teilweise eine Kollision in der Rutschbewegung anschloss (64\%). Zu einer Kollision aus der aufrechten Fahrbewegung heraus, ohne dass zuvor ein Sturz vorgelegen hatte, kam es in $36 \%$ der Fälle. 
Unter Berücksichtigung der am Unfallort vorgefundenen Spurenlage wurden darüber hinaus die Geschwindigkeiten der unfallbeteiligten Fahrzeuge überschlägig rekonstruiert. Mittels der Schadenbilder an den unfallbeteiligten Fahrzeugen sowie durch die Analyse der postkollisionären Auslaufphasen wurden die Kollisionsgeschwindigkeiten eingegrenzt. Lag darüber hinaus eine vorkollisionäre Spurenzeichnung an der Unfallörtlichkeit vor, konnte auch eine Rekonstruktion der Ausgangsgeschwindigkeit erfolgen. Kam es lediglich zu einem Sturz ohne Kollision, war auch hier eine Rekonstruktion der Ausgangsgeschwindigkeiten in Abhängigkeit der Spurenlage am Unfallort möglich.

In dem nachfolgenden Diagramm sind die ermittelten Ausgangs- und Kollisionsgeschwindigkeiten der unfallbeteiligten Krafträder bei den analysierten Alleinunfällen aufgezeigt.

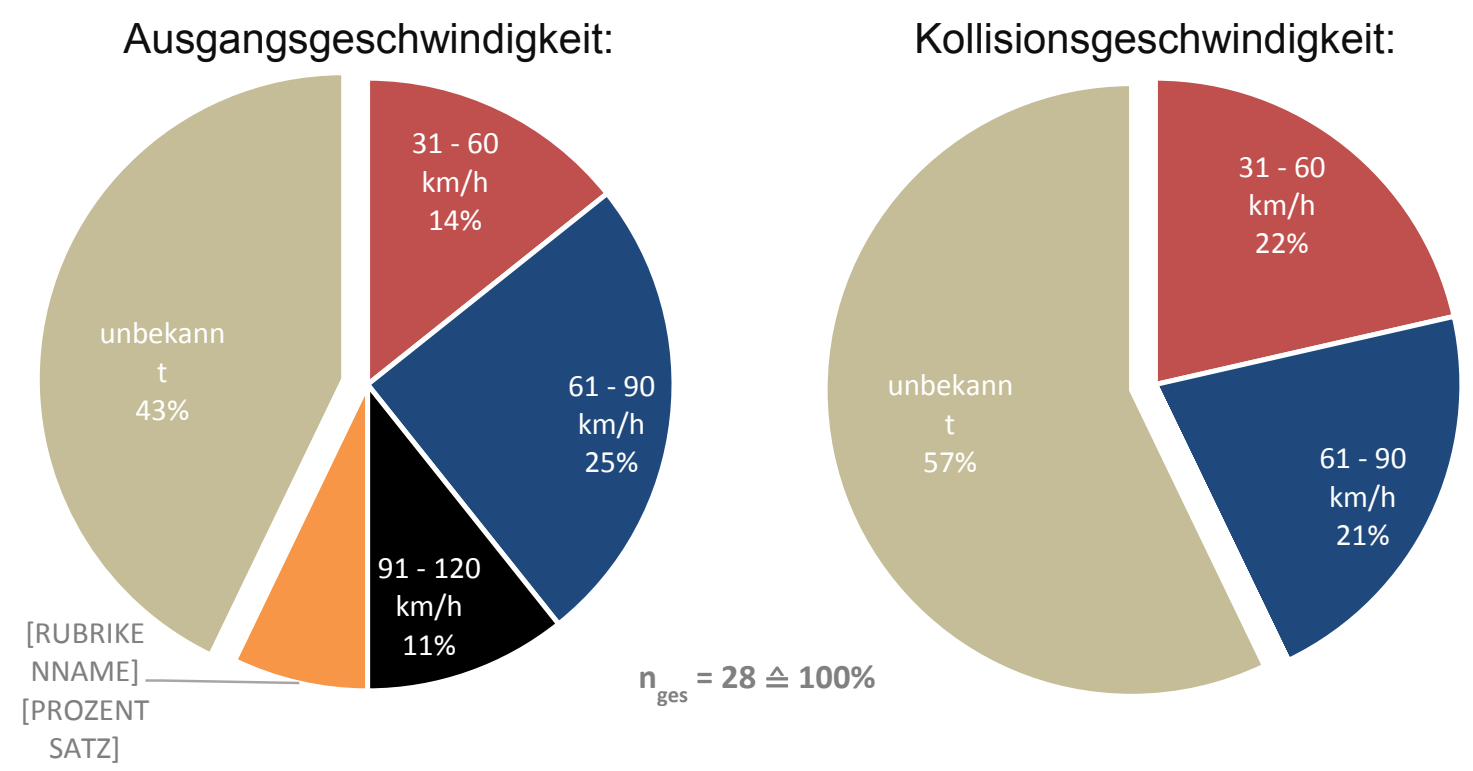

Diagramm 36:
Prozentuale Verteilung der Alleinunfälle nach Geschwindigkeiten der Krafträder

Die rekonstruierten Kollisionsgeschwindigkeiten der Krafträder lagen bei den Alleinunfällen im Bereich zwischen 31 und $90 \mathrm{~km} / \mathrm{h}$, die Ausgangsgeschwindigkeiten im Bereich von 31 bis 150 $\mathrm{km} / \mathrm{h}$. Die Durchschnittsgeschwindigkeit zum Zeitpunkt der Kollision ergibt sich mit $62,5 \mathrm{~km} / \mathrm{h}$.

\subsection{Vertiefende Analysen bei Unfallverursachung durch den Kradfahrer}

Werden die von Kradfahrern verursachten Unfälle betrachtet, so ergibt sich ein Schwerpunkt bei den Unfallarten 1 (Zusammenstoß mit einem anderen Fahrzeug, das anfährt, anhält oder im ruhenden Verkehr steht) sowie 2 (Zusammenstoß mit einem anderen Fahrzeug, das vorausfährt oder wartet).

In diesen Fällen ereigneten sich die Unfälle überwiegend (ca. 57\%) innerorts. Meist handelte es sich bei der jeweiligen Unfallörtlichkeit nicht um Einmündungs- bzw. Kreuzungsbereiche, sondern um Strecken ohne Knotenpunkte, deren Verkehrsfluss beispielsweise durch am Fahrbahnrand geparkte Fahrzeuge beeinflusst wurde. Bei nahezu der Hälfte der analysierten Verkehrsunfälle (ca. 43\%) kam es zunächst zu einem Sturz des Krades vor dem Hauptunfallereignis (Kollision). 
Werden die entsprechenden Verkehrsunfälle nach der jeweiligen Unfallursache unterschieden, so zeigt sich, dass sich mehr als jeder dritte Unfall (35\%) auf Grund von ungenügendem Sicherheitsabstand ereignet hatte. Im nachfolgenden Diagramm ist die prozentuale Verteilung der Unfallursachen dargestellt.

\section{Unfallursache}

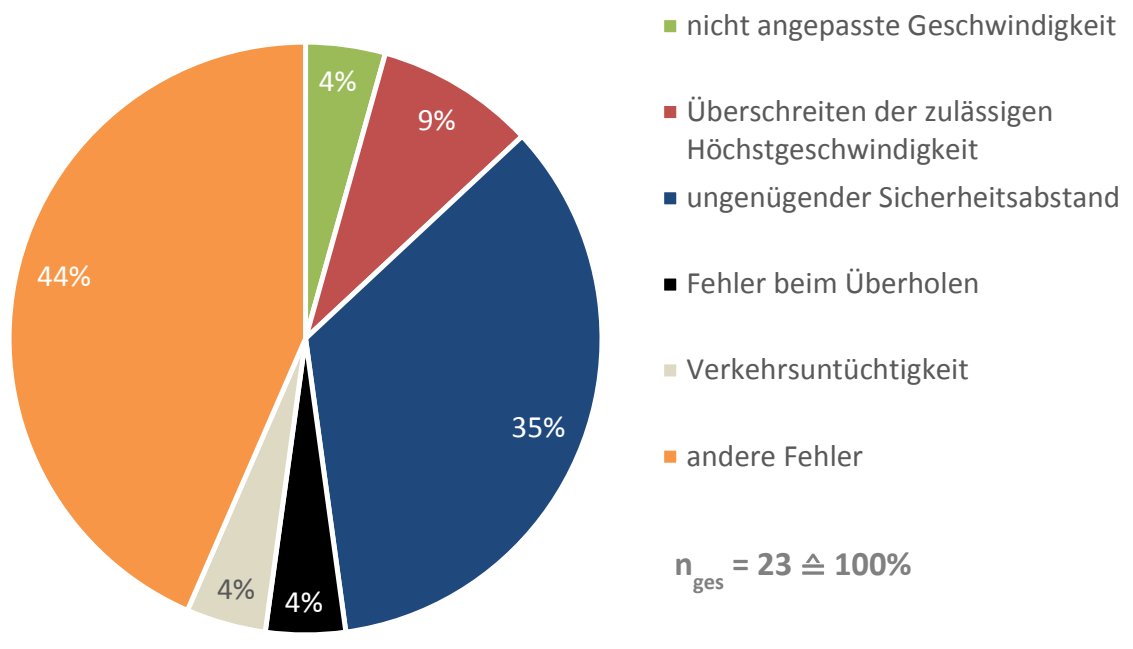

\section{Diagramm 37:}

Prozentuale Verteilung der durch den Kradfahrer verursachten Verkehrsunfälle nach Unfallursache

Wird ferner der jeweilige Unfallmechanismus der untersuchten Verkehrsunfälle näher betrachtet, so wird deutlich, dass in der Regel vor dem eigentlichen Unfallereignis eine sogenannte Abwehrhandlung des Kradfahrers nachvollzogen werden konnte. Hierbei handelte es sich im Wesentlichen um die Einleitung eines Bremsvorganges, teilweise in Kombination mit einem Ausweichvorgang. Es ergibt sich die im Diagramm 38 wiedergegebene Verteilung.

\section{Reaktion vor Unfallereignis}

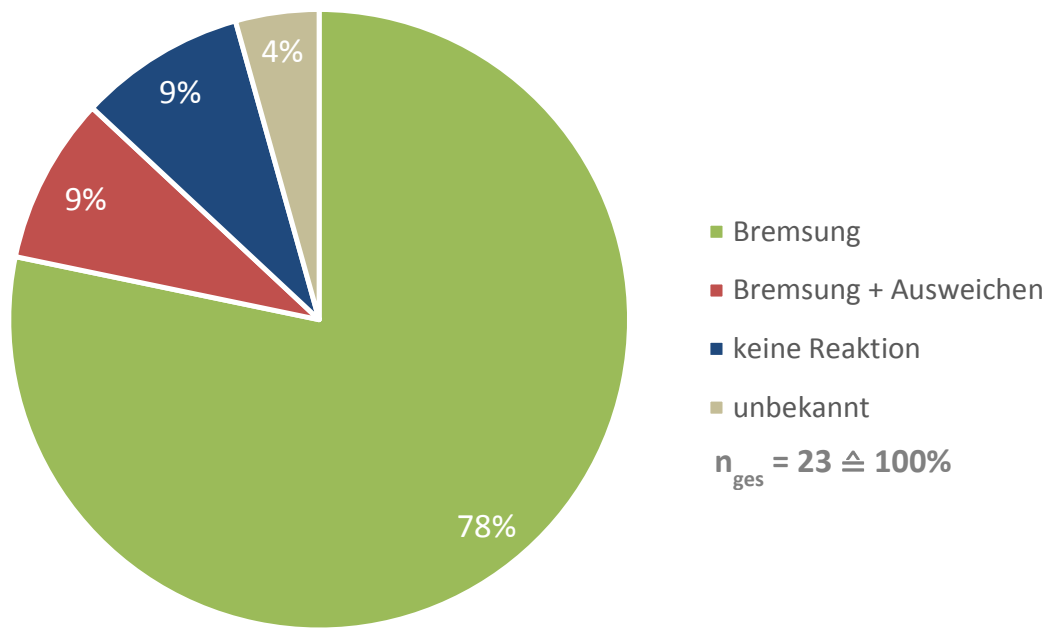

Diagramm 38:

Prozentuale Verteilung der untersuchten, vom Kradfahrer verursachten Unfälle nach Reaktion vor Unfallereignis 
Im nachfolgend dargestellten Diagramm sind die rekonstruierten Ausgangs- und Kollisionsgeschwindigkeiten der unfallbeteiligten Krafträder bei den analysierten Verkehrsunfällen, die durch den Kradfahrer verursacht wurden, dargestellt.

Ausgangsgeschwindigkeit:

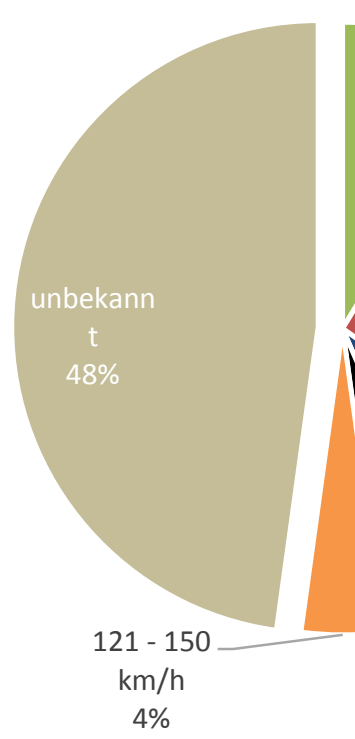

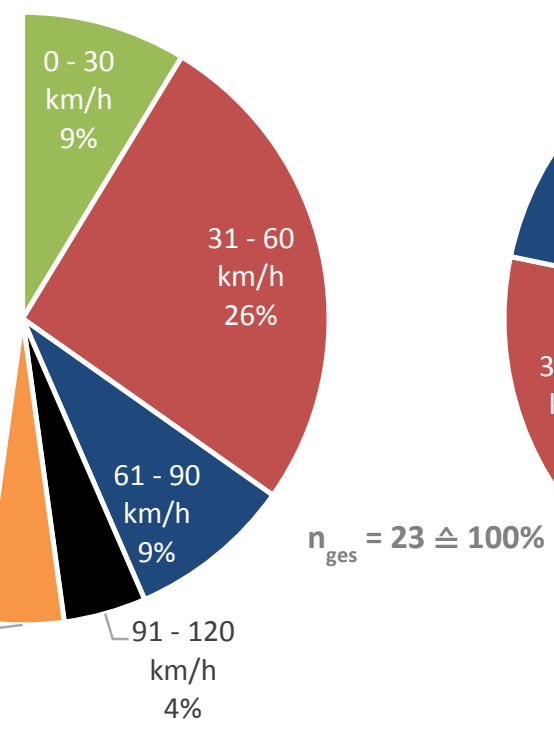

Kollisionsgeschwindigkeit:

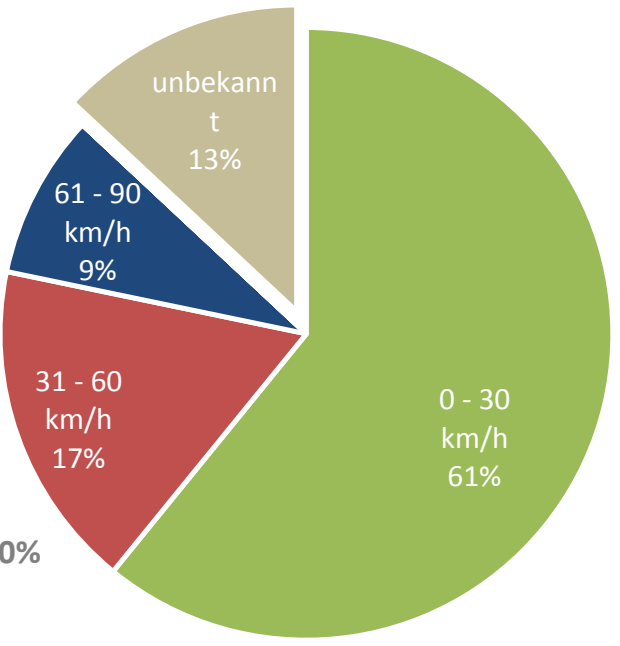

Diagramm 39:

Prozentuale Verteilung der vom Kradfahrer verursachten Unfälle nach Geschwindigkeitsniveau der Krafträder

Es zeigt sich, dass die Kollisionsgeschwindigkeiten überwiegend im Bereich von bis $\mathrm{zu} 60 \mathrm{~km} / \mathrm{h}$ lagen (78\%). Die durchschnittliche Kollisionsgeschwindigkeit ergibt sich mit 25,8 km/h.

\subsection{Vertiefende Analysen bei Unfallverursachung durch den Unfallgegner}

Wurden die im Erhebungszeitraum analysierten Verkehrsunfälle durch den Unfallgegner verursacht, so handelte es sich hierbei überwiegend um Zusammenstöße des Kraftrades mit einem anderen Fahrzeug, das abbiegt, einbiegt oder kreuzt (Unfallart 5, $n=54$, ca. 52\%).

Etwa zwei Drittel dieser Unfälle ereigneten sich innerorts. Zudem handelte es sich beim Unfallort im Wesentlichen um Einmündungs- bzw. Kreuzungsbereiche (ca. 82\%).

Die Auswertung der Ursachen der aufgenommenen Verkehrsunfälle verdeutlicht, dass diese in der Regel auf Grund von Fehlern beim Abbiegen, Wenden, Einfahren bzw. durch die Missachtung der Vorfahrtsregelung, entstanden waren. Im nachfolgenden Diagramm ist die prozentuale Verteilung der Unfallursachen schematisch dargestellt. 


\section{Unfallursache}

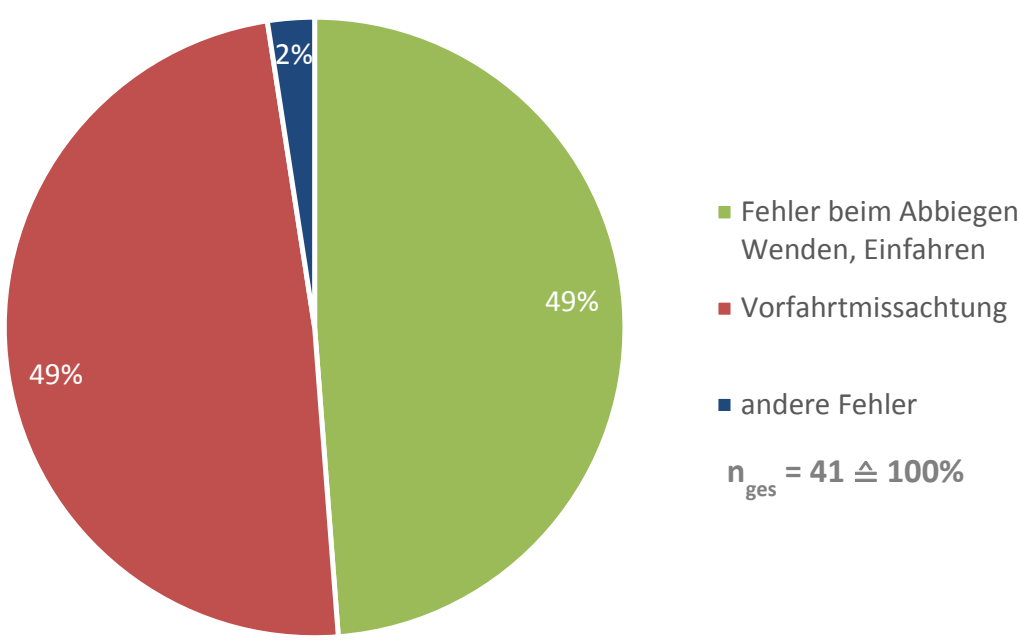

Diagramm 40:

Prozentuale Verteilung der Verkehrsunfälle, die durch den Unfallgegner verursacht wurden, nach entsprechender Ursache

In ca. 57\% der entsprechenden Fälle konnte vor dem jeweiligen Unfallereignis eine sogenannte Abwehrhandlung des Kradfahrers in Form eines Bremsvorganges nachvollzogen werden. In den übrigen Fällen konnte im Zuge der jeweiligen Unfallrekonstruktion keine Reaktion vor dem Unfallereignis nachvollzogen werden.

Werden die untersuchten Unfälle weiter im Hinblick auf den Unfallablauf bzw. Unfallmechanismus des Zweirades untersucht, so zeigt sich, dass die Zweiräder überwiegend (ca. 74\%) in aufrechter Position mit dem Unfallgegner kollidiert waren. Zu einem vorkollisionären Sturz des Kraftrades kam es in ca. $26 \%$ der berücksichtigten Fälle.

In dem nachfolgenden Diagramm sind die rekonstruierten Ausgangs- und Kollisionsgeschwindigkeiten der unfallbeteiligten Krafträder (Unfallart 5), wiedergegeben.

Ausgangsgeschwindigkeit:

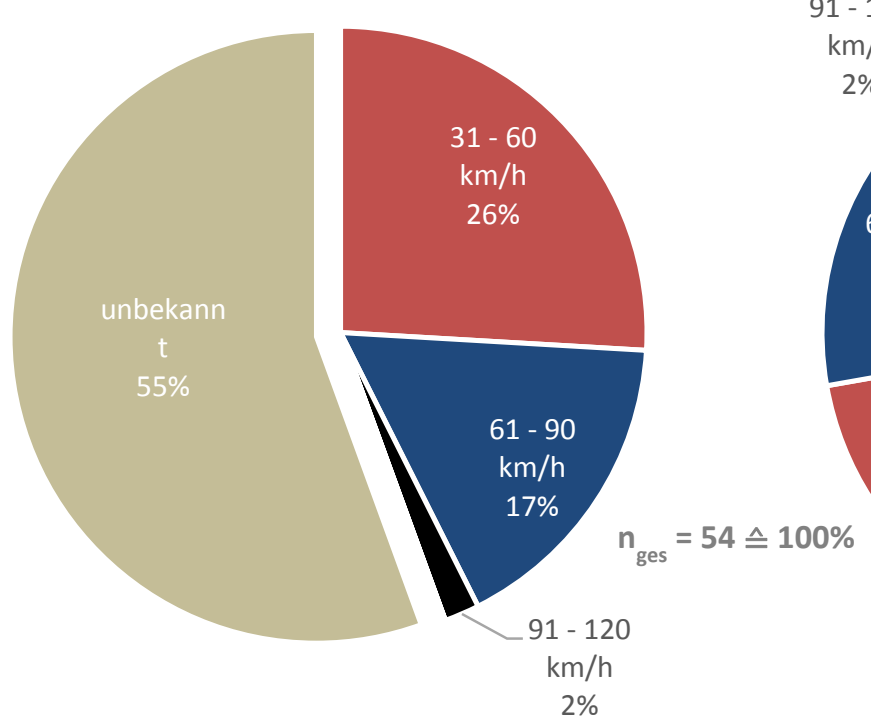

Kollisionsgeschwindigkeit:

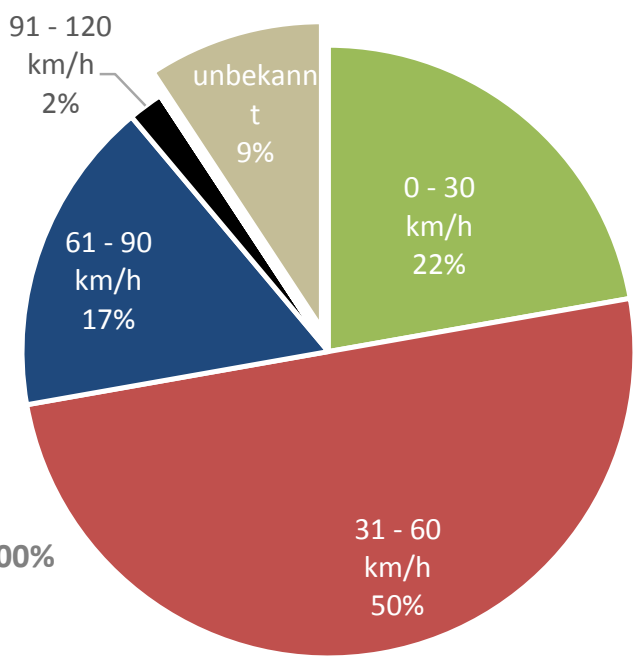

Diagramm 41:

Prozentuale Verteilung der durch den Unfallgegner verursachten Unfälle nach Geschwindigkeiten der Krafträder 
Die Rekonstruktion zeigt, dass die Krafträder in etwa der Hälfte der Fälle Kollisionsgeschwindigkeiten im Bereich von $31-60 \mathrm{~km} / \mathrm{h}$ innehatten. Die Durchschnittsgeschwindigkeit zum Zeitpunkt der Kollision ergibt sich zu 46,4 km/h.

\section{Auswertungen zu Unfällen bei schwer-bzw. schwerstverletzten Kradfahrern}

Die im Erhebungszeitraum aufgenommenen und analysierten Verkehrsunfälle wurden auch im Hinblick auf den Verletzungsgrad der Kradfahrer weitergehend ausgewertet. Insbesondere erfolgte eine Analyse der Verkehrsunfälle, bei denen besonders schwerwiegende Verletzungen (MAIS 3+) der Kradfahrer eingetreten waren $(n=22)$. In diesen Fällen wurden somit die Maximalwerte der Einzelverletzungen nach der AIS-Codierung zwischen 3 und 6 berücksichtigt.

Es konnte festgestellt werden, dass es zu entsprechend schwerwiegenden Verletzungsbildern der Kradfahrer in ca. $64 \%$ der Fälle außerorts gekommen war. Wird ferner das Unfallverursachungsmuster analysiert, so zeigt sich, dass in ca. 55\% der entsprechenden Fälle von einer Verursachung durch den Unfallgegner auszugehen war. In ca. 18\% der Fälle wurden die Verkehrsunfälle durch den Kradfahrer verursacht. Bei dem Rest der Unfälle (ca. 27\%) handelte es sich um Alleinunfälle.

Bei nahezu zwei Drittel der Fälle (ca. 64\%) kollidierte das Kraftrad mit dem Unfallgegner in einer aufrechten Fahrbewegung. Vor dem Unfallereignis konnte eine Abwehrhandlung des Kradfahrers in nur etwa jedem zweiten Fall sicher nachvollzogen werden.

Im nachfolgend dargestellten Diagramm sind die ermittelten Ausgangs- und Kollisionsgeschwindigkeiten der unfallbeteiligten Krafträder bei den analysierten Verkehrsunfällen mit besonders schwerwiegenden Verletzungen der Kradfahrer (MAIS 3+) wiedergegeben.

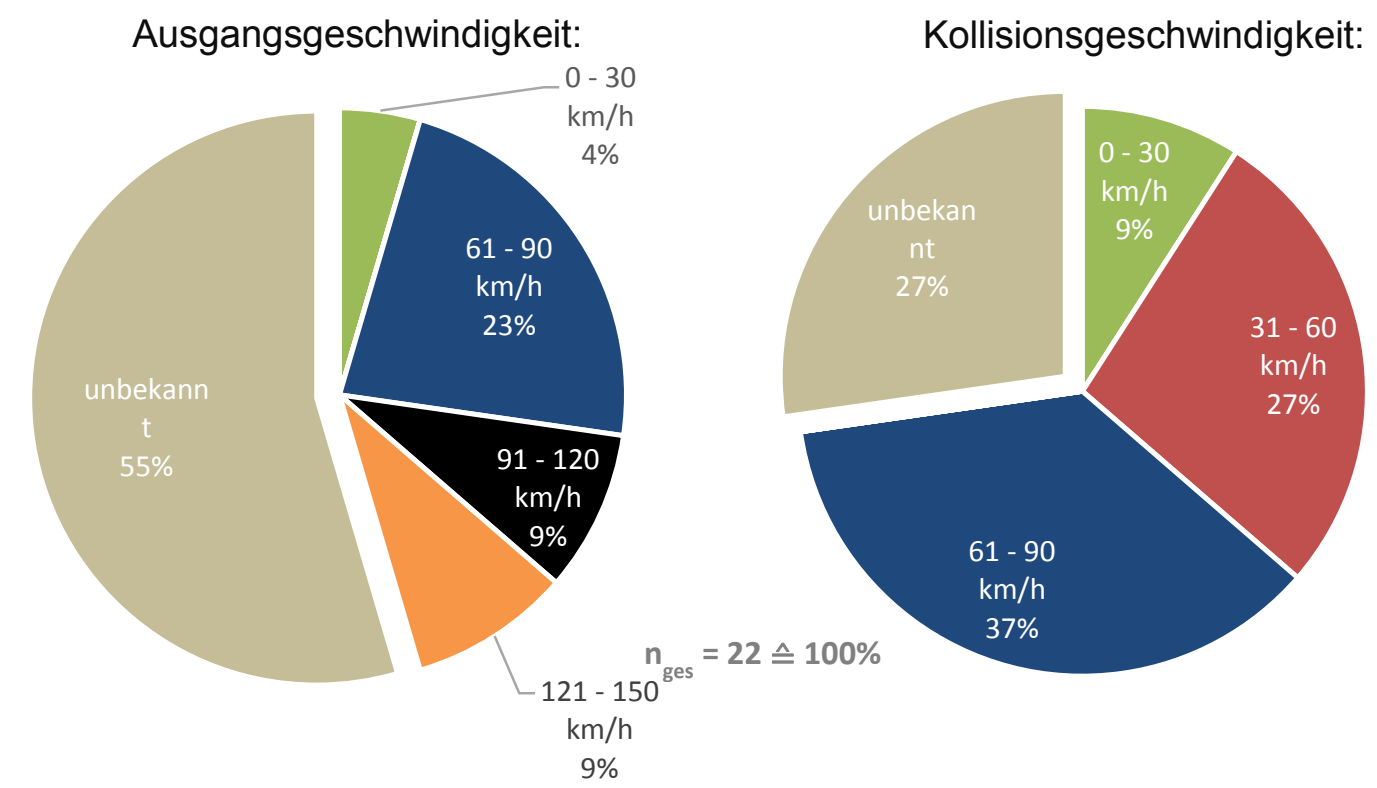

Diagramm 42:

Prozentuale Verteilung der Verkehrsunfälle mit besonders schwerwiegenden Verletzungsmustern der Kradfahrer nach Geschwindigkeiten der Krafträder 
Das vorstehende Diagramm verdeutlicht, dass es besonders häufig zu schweren Verletzungen (MAIS 3+) der Kradfahrer in einem Kollisionsgeschwindigkeitsbereich von ca. 31 - $90 \mathrm{~km} / \mathrm{h}$ gekommen war. Der Durchschnitt der rekonstruierten Kollisionsgeschwindigkeiten ergibt sich mit $58,8 \mathrm{~km} / \mathrm{h}$.

Werden die entsprechenden, im Erhebungszeitraum aufgenommenen Verkehrsunfälle nach der Unfallart kategorisiert, so ergibt sich die im nachfolgend dargestellten Diagramm aufgezeigte prozentuale Verteilung.

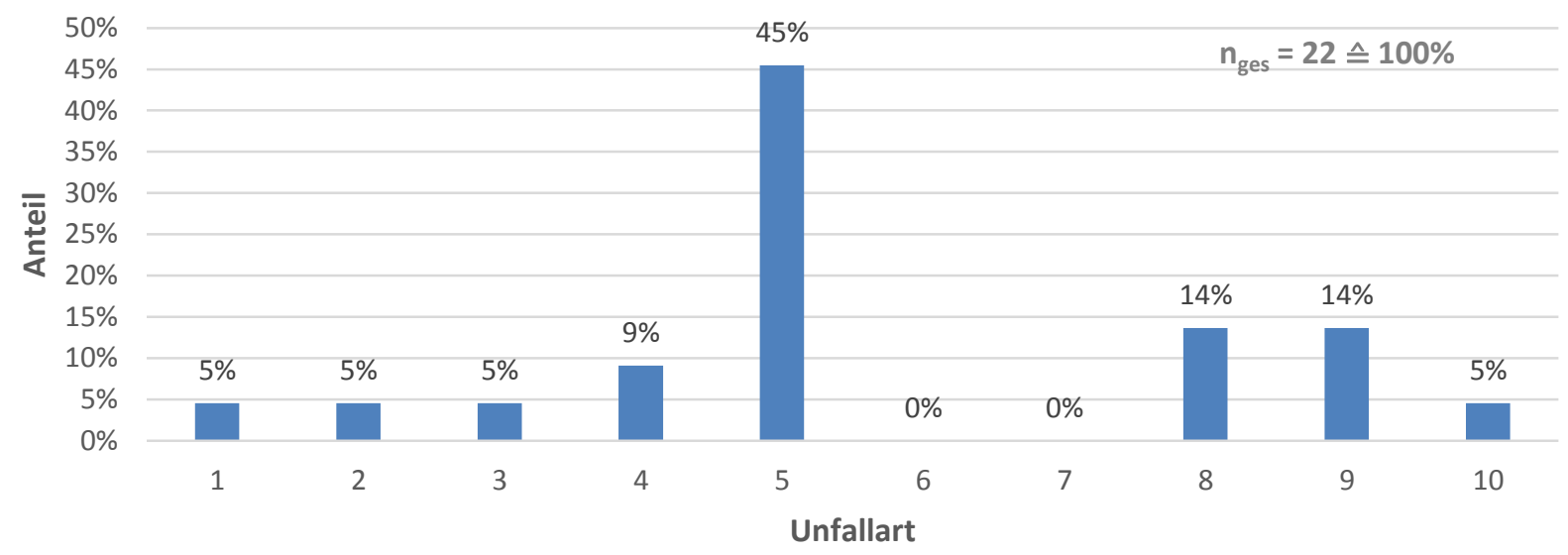

Diagramm 43:

Prozentuale Verteilung der untersuchten Verkehrsunfälle mit besonders schwerwiegenden Verletzungen der Kradfahrer (MAIS 3+) nach Unfallart

Es wird ersichtlich, dass häufig Verkehrsunfälle, bei denen es zum Zusammenstoß mit einem anderen Fahrzeug kam, das abbog, einbog oder kreuzte (Unfallart 5), zu besonders schwerwiegenden Verletzungen der Kradfahrer führten (45\%).

Werden die entsprechenden Verkehrsunfälle nach dem Unfalltyp unterschieden, so ergibt sich das nachfolgend dargestellte Verteilungsmuster.

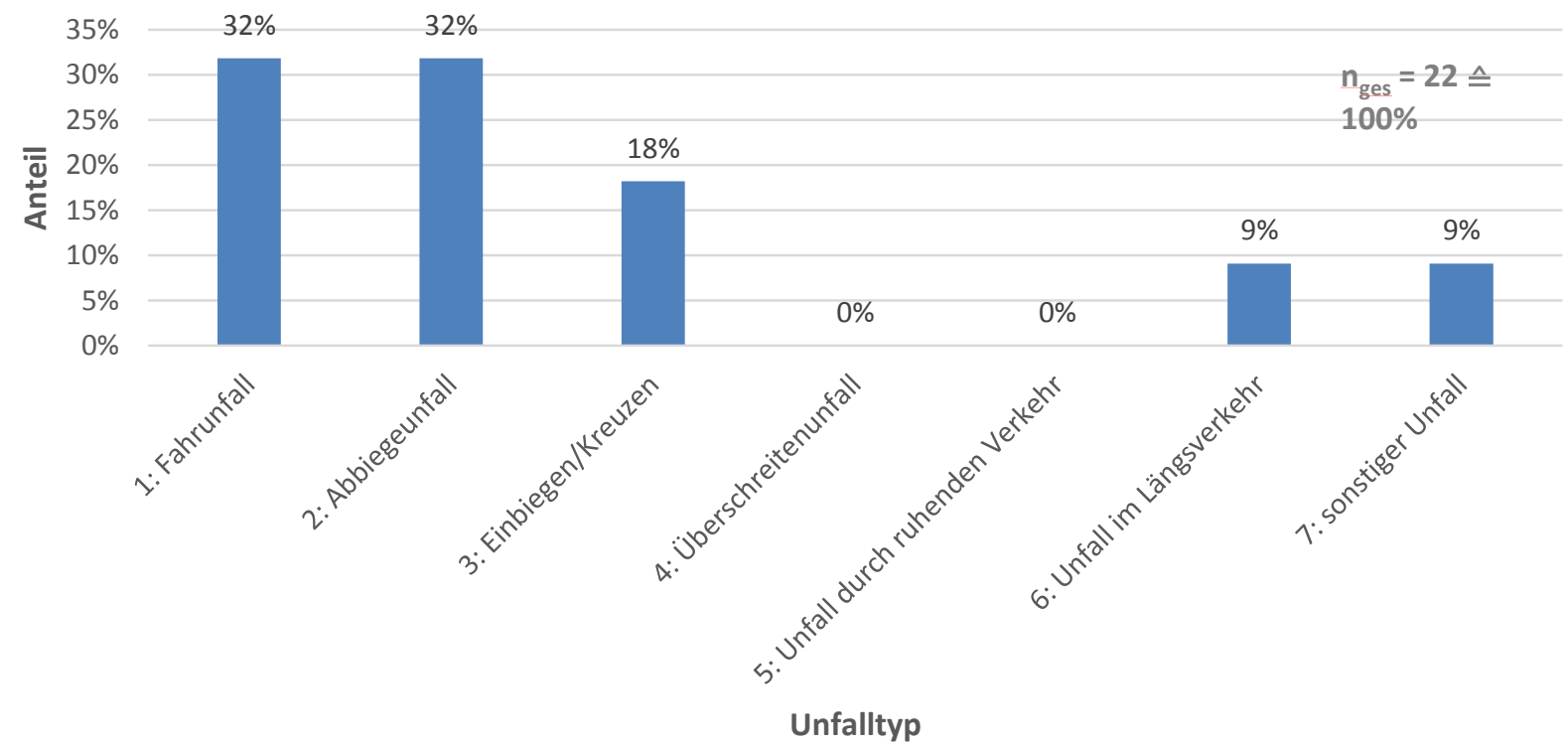

Diagramm 44:

Prozentuale Verteilung der untersuchten Verkehrsunfälle mit besonders schwerwiegenden Verletzungen der Kradfahrer (MAIS 3+) nach Unfalltyp 
Im Gebiet des Saarlandes konnten durch die Unfallforschungsteams im Zeitraum von Mai 2010 bis Dezember 2011 insgesamt 194 Verkehrsunfälle unter der Beteiligung von Krafträdern unmittelbar nach dem Unfall in Augenschein genommen und weitergehend ausgewertet werden.

Besonders häufig kam es zu Verkehrsunfällen unter der Beteiligung von sogenannten Naked-Bikes bzw. Sportlern sowie Sporttourern. Mehr als die Hälfte aller aufgenommenen Verkehrsunfälle ereignete sich an den Tagen des Wochenendes (Freitag bis Sonntag). Überwiegend kam es zu Verkehrsunfällen im Zeitraum zwischen 12 und 18 Uhr. In der Regel ereigneten sich die Verkehrsunfälle bei Tageslicht sowie trockener Fahrbahnoberfläche. Es konnte weiter festgestellt werden, dass es sich bei ca. einem Fünftel der aufgenommenen Verkehrsunfälle um Alleinunfälle ohne die Beteiligung anderer Verkehrsteilnehmer gehandelt hatte. Sofern ein Unfallgegner vorhanden war, handelte es sich bei diesen in der Regel um einen Pkw. Etwa zwei Drittel der Unfälle, bei denen es sich nicht um Alleinunfälle handelte, wurden durch den Unfallgegner verursacht.

Wird nach der Unfallart unterschieden, so zeigt sich ein Schwerpunkt bei den Verkehrsunfällen, bei denen es zu einem Zusammenstoß mit einem anderen Fahrzeug im abbiegenden, einbiegenden bzw. kreuzenden Verkehr gekommen war (Unfallart 5, ca. 31\%).

Im Hinblick auf die Sicherheitsausstattung der unfallbeteiligten Krafträder zeigte sich, dass weniger als jedes fünfte, im Erhebungszeitraum verunfallte Kraftrad mit einem sogenannten Antiblockiersystem ausgestattet war.

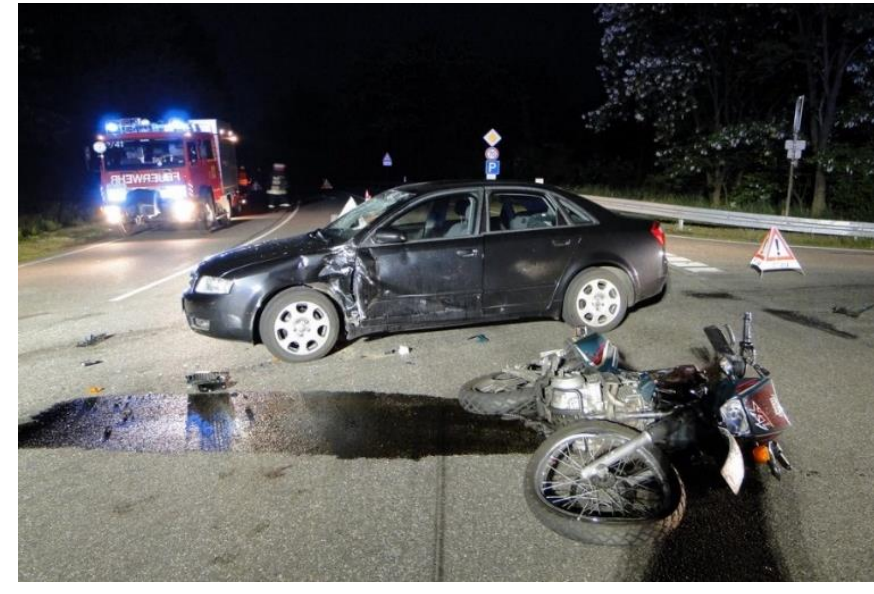

Abbildung 5:

Einbiegen/Kreuzen-Unfall

Etwa jeder dritte verunfallte Zweiradfahrer war zwischen 41 und 50 Jahren alt. Weiterhin zeigte sich ein hoher Anteil verunfallter Zweiradfahrer von etwa $26 \%$ in der Altersgruppe von 18- bis 30 Jahren.

Eine nachvollziehbare Einschränkung der Fahrtüchtigkeit der Zweiradfahrer ergab sich in ca. 2\% der Fälle. Diese Fahruntüchtigkeit konnte überwiegend auf Alkoholeinfluss zurückgeführt werden.

Im Erhebungszeitraum wurden insgesamt neun Zweiradaufsassen tödlich verletzt. Nahezu die Hälfte aller beteiligten Zweiradaufsassen erlitt schwere Verletzungen. Die Analyse der durch die verunfallten Zweiradaufsassen getragenen Bekleidung verdeutlicht, dass überwiegend Integralschutzhelme (ca. 83\%) getragen wurden. In der Mehrzahl trugen die Kradfahrer spezielle Motorradschutzkleidung. Lediglich im Hinblick auf das Schuhwerk konnte festgestellt werden, dass nur unwesentlich mehr als jeder zweite Kradfahrer spezielle Motorradschutzstiefel getragen hatte. 


\section{Besondere Feststellungen nach Unfallverursachungsmuster}

Die aufgenommenen Verkehrsunfälle wurden zur weitergehenden Auswertung in die nachfolgend aufgeführten Kategorien unterteilt:

- $\quad$ Alleinunfälle $(n=40)$

- Kradfahrer als Unfallverursacher $(n=50)$

- Unfallgegner als Unfallverursacher $(n=104)$

Im Hinblick darauf konnte bei den Alleinunfällen besonders häufig die Beteiligung von sogenannten Naked-Bikes bzw. Sportlern festgestellt werden. Etwa jeder zweite Alleinunfall ereignete sich am Wochenende (Samstag und Sonntag). Überwiegend kam es zu den entsprechenden Unfällen außerorts (auch auf Autobahnen). Bemerkenswert hoch ist zudem der Anteil der Alleinunfälle in der Altersgruppe der 21- bis 30-jährigen Zweiradfahrer. Wird ferner eine Aggregation der Einzelverletzungen nach der Abbreviated Injury Scale (AIS) durchgeführt, so zeigt sich, dass es beim Alleinunfall überwiegend zu schwerwiegenden Verletzungen (MAIS 2+) gekommen war. In der Regel kam es beim Alleinunfall zu einem Abkommen von der Fahrbahn (Unfallarten 8 und 9). Unfallursächlich war in den meisten Fällen eine nicht angepasste Geschwindigkeit, teilweise auch mit einem Überschreiten der örtlich zulässigen Höchstgeschwindigkeit.

Auch bei Unfällen mit mehreren Beteiligten, die durch den Kradfahrer verursacht wurden, konnte festgestellt werden, dass häufig die Motorrad-Bauformen Naked-Bike und Sportler beteiligt waren. Im Hinblick auf das Alter der unfallbeteiligten Kradfahrer zeigte sich ein überwiegender Anteil der 41- bis 60-Jährigen. Bei den Unfallkonstellation lag der Schwerpunkt bei den sogenannten Auffahrkollisionen (Unfallarten 1 und 2).

Zu Verkehrsunfällen, die durch den Unfallgegner (in der Regel Pkws) verursacht wurden, kam es hauptsächlich innerorts und im Zeitraum von Montag bis Freitag. Zudem lag der Unfallort im Wesentlichen im Einmündungs- und Kreuzungsbereich. Ferner konnte festgestellt werden, dass sich als Unfallursachenschwerpunkte insbesondere Fehler beim Abbiegen, Wenden oder Einfahren sowie die Missachtung der Vorfahrtsregelungen herausgestellt haben.

Werden alle schwerverletzten Zweiradaufsassen nach polizeilicher Erhebung berücksichtigt, so ergibt sich ein Anteil polytraumatisierter Zweiradaufsassen mit einem ISS $\geq 16$ von ca. 10\%.

\section{Unfälle mit schwer- bzw. schwerstverletzten Kradfahrern}

Besonders schwerwiegende Verletzungsbilder (MAIS 3+) der Kradfahrer konnten überwiegend bei Verkehrsunfällen festgestellt werden, die sich außerorts ereignet hatten. Mehr als die Hälfte der entsprechenden Verkehrsunfälle wurden durch den Unfallgegner verursacht. In ca. 64\% der Fälle kam es zu einer Kollision des Kraftrades mit dem Unfallgegner während einer aufrechten Fahrbewegung. Eine vorherige Abwehrhandlung des Kradfahrers (z. B. Bremsung, Ausweichbewegung) konnte in nur etwa jedem zweiten Fall nachvollzogen werden.

Wie die Unterscheidung nach der Unfallart verdeutlicht, kam es bei nahezu jedem zweiten Unfall zu einem Zusammenstoß mit einem anderen Fahrzeug im abbiegenden, einbiegenden oder kreuzenden Verkehr. 
Zur Erhöhung der Sicherheit im Straßenverkehr ist es erforderlich, die Zahl der Verkehrsunfälle dauerhaft und konsequent zu senken. Aber auch in den Situationen, in denen ein Unfall weiterhin unabwendbar ist, sind Maßnahmen erforderlich, die dazu beitragen, das Unfallausmaß und somit auch das Verletzungsrisiko zu reduzieren.

Die Auswertungen der erhobenen Verkehrsunfälle verdeutlichen besonders häufige Unfallkonstellation sowie Problemstellungen. Besonders häufig kam es hier zu Zusammenstößen, bei denen ein anderer Verkehrsteilnehmer abbog, einbog oder kreuzte. Ging die Unfallverursachung vom Kradfahrer aus, so stellten sich diese Unfälle gehäuft als Alleinunfälle (z. B. Abkommen von der Fahrbahn) oder aber als Auffahrunfälle auf vorausfahrende Fahrzeuge heraus. Signifikant ist weiterhin, dass offensichtlich ABS bei Krafträdern noch immer selten im Straßenverkehr anzutreffen ist. Nachfolgend wird weiter auf die bedeutendsten Gegenmaßnahmen eingegangen.

\section{Technische Maßnahmen}

Wie bereits dargelegt, ereignete sich ein großer Teil der aufgenommenen Verkehrsunfälle bei Abbiege-, Einbiegevorgängen bzw. im kreuzenden Verkehr. Hierbei wurden die Krafträder teilweise gänzlich vom Unfallgegner übersehen bzw. zu spät erkannt. Eine deutlich bessere Erkennbarkeit der Zweiradaufsassen kann durch das Tragen von Warnwesten in auffälliger Farbgebung mit fluoreszierenden und retroreflektierenden Flächen sowohl bei Tageslicht als auch bei Dunkelheit ermöglicht werden. Allerdings werden entsprechende Westen derzeit nur sehr selten von Motorradaufsassen im Straßenverkehr getragen.

Zusätzlich wäre es auch denkbar, die Erkennbarkeit des Kraftrades selbst weiter zu verbessern. Möglicherweise könnten hierfür eine Umgestaltung der Fahrzeugsilhouette oder auch Änderungen bzw. Verbesserungen der lichttechnischen Einrichtungen sorgen.

\section{ABS}

Um Motorradunfälle zu reduzieren bzw. zu vermeiden, ist eine schnelle Einführung zusätzlicher technischer Assistenzsysteme sowie die Weiterentwicklung von bereits vorhandenen technischen Assistenzsystemen erforderlich. Insbesondere wird hier auf die Einführung von ABS mit Schräglagenerkennung sowie Abstandsradarsysteme, die einen ausreichenden Sicherheitsabstand sicherstellen, verwiesen.

Die Auswertungen der aufgenommenen Verkehrsunfälle zeigen, dass weniger als jedes fünfte unfallbeteiligte Kraftrad mit einem konventionellen Antiblockiersystem ausgestattet war. Im Zuge der Rekonstruktion der einzelnen Unfallabläufe konnte festgestellt werden, dass in ca. $44 \%$ aller Fälle $\left(n_{\text {gesamt }}=194\right)$ der Kradfahrer zu Fall gekommen war, ohne dass eine Kollision vorangegangen bzw. sturzursächlich war. In insgesamt 31\% der Fälle ( $n=60)$ konnte auf Grund der Spurenlage vor Ort nachvollzogen werden, dass unmittelbar vor dem Sturz eine starke Bremsverzögerung vorgelegen hatte, die mit dem Blockieren von zumindest einem Rad des Kraftrades einherging und somit sturzursächlich war. 
Diese Fälle $(n=60)$ wurden weitergehend analysiert, um Aussagen über das Potenzial von herkömmlichem ABS zur Minimierung des Unfallrisikos bzw. Unfallausmaßes zu treffen. Es zeigte sich, dass bei nahezu jedem der betrachteten Fälle (ca. 95\%, $n=57$ ) das Vorhandensein eines Antiblockiersystems einen nennenswerten positiven Einfluss auf das Unfallgeschehen gehabt hätte.

Bei ca. $60 \%$ der ausgewerteten Fälle $(n=36$; entspricht ca. $19 \%$ aller aufgenommenen Verkehrsunfälle) wurde nach entsprechender Unfallrekonstruktion festgestellt, dass das Unfallgeschehen durch ein mit herkömmlichem ABS (ohne Schräglagenerkennung) ausgestattetes Kraftrad gänzlich zu vermeiden gewesen wäre. In weiteren $35 \%$ der berücksichtigten Fälle ( $n=21$; entspricht ca. $11 \%$ der Gesamtzahl der aufgenommenen Verkehrsunfälle) ist zumindest davon auszugehen, dass das Unfallausmaß und damit auch insbesondere das Verletzungsrisiko des Kradfahrers durch $A B S$ zu reduzieren gewesen wäre. In der Summe wären $30 \%(n=57)$ aller Unfälle durch ein mit einem herkömmlichen ABS ausgestatteten Kraftrad vermeidbar oder positiv beeinflussbar gewesen.

\section{eCall}

Bei eCall (Emergency Call) handelt es sich um ein System, dass im Falle eines Unfalls selbstständig durch entsprechend auslösende Sensoren oder manuell ausgelöst über das Mobilfunknetz die jeweilige örtliche zuständige Notrufabfragestelle informiert. Hierbei werden automatisch auch beispielsweise die Positionsdaten übermittelt. Darüber hinaus wird eine Sprechverbindung zwischen dem Unfallbeteiligten und der Rettungsleitstelle aufgebaut, um weitere Informationen zum Unfall und insbesondere auch dem Verletzungsbild des Unfallbeteiligten zu erhalten.

Gerade dann, wenn es auf Grund der Unfallschwere den Unfallbeteiligten nicht mehr möglich ist, selbstständig einen entsprechenden Notruf zu tätigen und entsprechende Informationen zum Unfall zu ermitteln, ist durch eCall sichergestellt, dass durch die Rettungsleitstelle eine sofortige Hilfeleistung veranlasst wird.

Das eCall-System soll voraussichtlich in allen EU-Staaten ab dem 31.03.2018 für Pkws und leichte Nutzfahrzeuge bis 3,5 t Leergewicht bei Neufahrzeugen Pflicht werden. Eine entsprechende Vorschrift für Krafträder existiert bisher nicht. Bereits im Januar 2013 fand die Markteinführung eines nachrüstbaren eCall-Systems für Motorräder in Deutschland, Österreich und der Schweiz statt. Das entsprechende System bestand aus einer Einheit, die am Motorradlenker angebracht wurde und einer weiteren Einheit, die am Helm anzubringen war. Durch die verbaute Sensorik konnten die Fahrbedingungen überprüft werden und im Bedarfsfall ein Notruf ausgelöst werden. Zwischenzeitlich wurde das System allerdings wieder vom Markt genommen. Um eine Potentialabschätzung des eCall-Systems bei Motorradunfällen abgeben zu können, wurden alle im Forschungsprojekt aufgenommenen Alleinunfälle unter einer Vielzahl von relevanten Gesichtspunkten untersucht.

Insbesondere wurden hierbei folgende Faktoren berücksichtigt:

- Verletzungsschwere

- Ortslage

- Unfallzeit bzw. Lichtverhältnisse

- zu erwartendes Verkehrsaufkommen 
- Vorhandensein möglicher Unfallzeugen

- Erkennbarkeit des Kradfahrers bzw. Krades für vorbeifahrende Fahrzeuge

eCall wurde dann als sinnvoll bzw. hilfreich angesehen, wenn schwerwiegende Verletzungen (MAIS $3+$ ) vorgelegen haben und beispielsweise von einem geringen Verkehrsaufkommen oder aber schlechter Erkennbarkeit der Unfallsituation für andere Verkehrsteilnehmer (z. B. Endlage des Kradfahrers am Fahrbahnrand nur schwer erkennbar) ausgegangen werden konnte. Grundsätzlich ist es auch denkbar, dass das Vorhandensein eines eCall-Systems bei weniger schwerwiegenden Verletzungsbildern (insbesondere MAIS 2) sinnvoll gewesen wäre. Konnte davon ausgegangen werden, dass andere Verkehrsteilnehmer unmittelbar nach dem Unfall Hilfe hätten leisten können oder war das Verletzungsbild des Kradfahrers so unerheblich, dass eine Hilfsanforderung auf eigenem Wege erfolgen konnte, ergäbe sich in derartigen Fällen keine erhebliche Verbesserung der Unfallsituation durch ein eCall-System.

Die Auswertungsergebnisse der aufgenommenen Verkehrsunfälle sind im nachfolgenden Diagramm schematisch wiedergegeben.

\section{Einfluss von eCall auf Alleinunfälle}

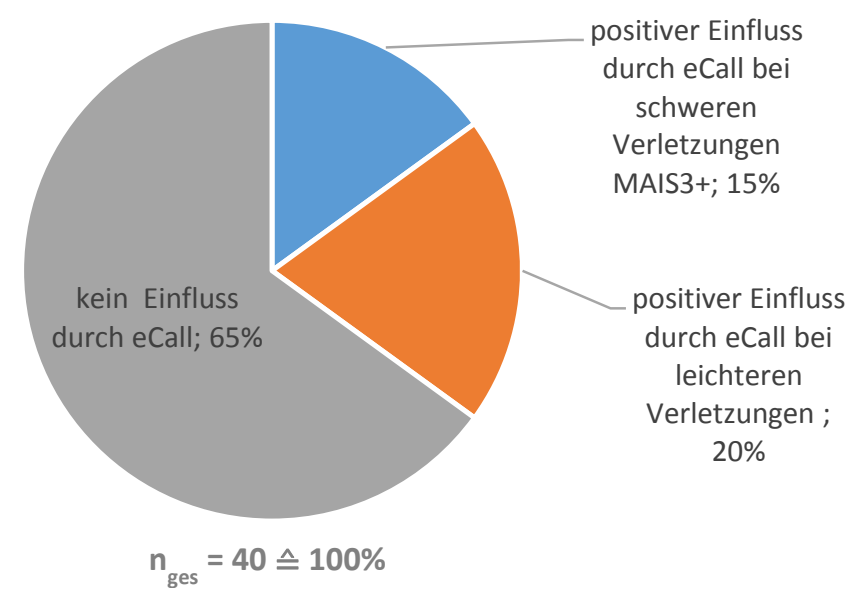

Diagramm 45:

Einfluss von eCall bei Alleinunfällen

Die Untersuchung der aufgenommenen Alleinunfälle verdeutlicht, dass 14 aller Alleinunfälle (entspricht $35 \%$ ) positiv durch eCall zu beeinflussen gewesen wären. Das sind $7 \%$ aller aufgenommenen Unfälle. Bei Betrachtung des Einflusses von eCall auf Unfälle mit schwerwiegenden Folgen (MAIS 3+) reduziert sich dieser Anteil auf 3\% aller aufgenommenen Unfälle.

Die Verbesserung der aktiven Fahrzeugsicherheit sollte sich auch auf die potentiellen Unfallgegner (insbesondere Pkw) ausweiten. Somit könnte sichergestellt werden, gewisse Unfallsituationen gänzlich zu vermeiden oder aber zumindest die Unfallfolgenschwere deutlich zu verringern. Hier wären Systeme zu nennen, die in der Lage sind, sicherheitskritische Informationen zwischen Verkehrsteilnehmern untereinander aber auch mit der Infrastruktur auszutauschen [13]. 


\section{Persönliches Verhalten}

Gerade Alleinunfälle werden durch den Kontrollverlust über das eigene Fahrzeug hervorgerufen. Den größten Einfluss üben hierauf die eigene Fahrweise und Risikobereitschaft des Kradfahrers aus. In diesem Zusammenhang ist auch darauf hinzuweisen, dass Geschwindigkeitsverstöße von Motorradfahrern deutlich schlechter kontrollierbar sind als beispielsweise von Pkw-Fahrern.

Regelmäßige Fahrertrainings können einerseits das persönliche Fahrvermögen verbessern und auch den Kradfahrer im Hinblick auf das eigene Gefahrenbewusstsein sensibilisieren.

\section{Infrastruktur}

Gerade auf Strecken, die bevorzugt von Motorrädern befahren werden, und von denen eine entsprechende Unfallgefahr ausgeht, könnte durch adäquate Maßnahmen für eine Steigerung der Sicherheit gesorgt werden. Auf unfallträchtigen, kurvigen Strecken haben sich beispielsweise Rüttelstreifen zur Reduzierung des Tempos bewährt [14]. Auch sollten die Fahrbahnoberflächen in einem derartigen Zustand (keine provisorischen Fahrbahnreparaturen oder Fahrbahnunebenheiten) sein, dass hiervon keine Gefahr für Motorradfahrer ausgeht.

Sollte es dennoch zu einem Sturz des Kradfahrers kommen, so sollte auch in diesen Fällen das mögliche Verletzungsrisiko für den Kradfahrer, z. B. durch Schutzplanken mit Unterfahrschutz, reduziert werden.

\section{Crashversuche Motorrad - Pkw}

Die Unfalldatenauswertung zeigt, dass fast jeder zweite Unfall, den ein Kradfahrer verursacht, ein sogenannter Unfall im Längsverkehr ist. Die typische Unfallkonstellation stellt hier der Auffahrunfall dar. Unzureichender Sicherheitsabstand des Motorradfahrers zum vorausfahrenden Fahrzeug oder aber auch eine kurzzeitige Ablenkung können hier unfallursächlich sein. Entsprechende Kollisionen führen häufig zu schweren Verletzungen der Kradfahrer.

Um weitere Erkenntnisse zur bislang unterschätzten Gefahr des Auffahrunfalles zu erlangen, wurden im Mai 2014 zwei Motorradcrashtests bei der Firma CTS in Münster durchgeführt. Die Tests waren durch die folgenden Randbedingungen gekennzeichnet:

- Auffahrkollision eines ungebremst auffahrenden Kraftrades auf das Heck eines Pkw, welcher aus einer Fahrbewegung heraus abgebremst wird

- Ausgangssituation: beide Fahrzeuge mit nahezu gleicher Ausgangsgeschwindigkeit hintereinander fahrend (ca. 60 bis $70 \mathrm{~km} / \mathrm{h}$ )

- im Anschluss daran starker Abbremsvorgang des Pkw, sodass es zur Kollision des Kraftrades mit dem Pkw kommt

- Aufprallgeschwindigkeit des Kraftrades zum Zeitpunkt der Kollision mit dem Pkw: ca. 30 $\mathrm{km} / \mathrm{h}$ höher als die des Pkw

- Bauart der Krafträder: sogenannte Naked-Bikes, mit einem Dummy (Typ: Hybrid II, nicht instrumentiert) besetzt

- Kollision des Kraftrades mit dem Pkw unter einem seitlichen Versatz nach rechts. 
Nachfolgend sind die Ausgangsparameter der Crashversuche schematisch dargestellt.

(1) $\quad \mathrm{v}_{\mathrm{AKrad}}=\mathrm{v}_{\mathrm{APkw}}=\mathrm{ca} .60-70 \mathrm{~km} / \mathrm{h}$
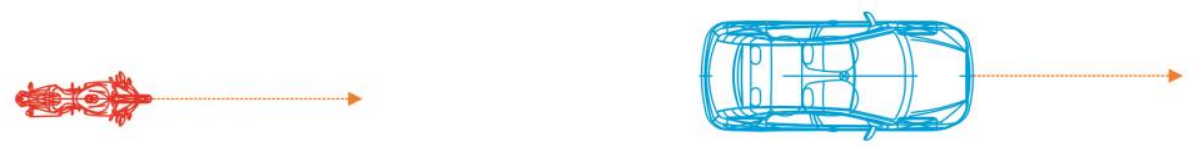

(2) $\quad \mathrm{v}_{\mathrm{Krad}}=\mathrm{v}_{\mathrm{A} \text { Krad }}=$ konst., während Pkw verzögert
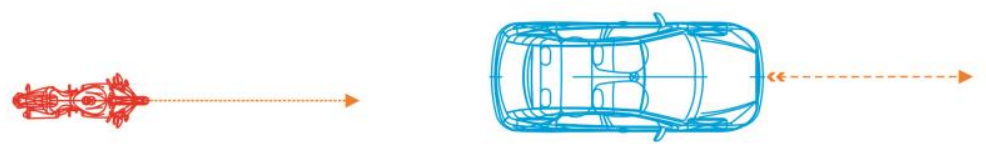

(3) Krad fährt auf verzögerten Pkw auf, $\Delta v$ ca. $30 \mathrm{~km} / \mathrm{h}$

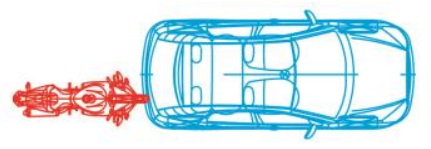

Abbildung 6:

Prinzipieller Ablauf des Crashversuchs

Bei den zu den Crashversuchen herangezogenen Pkws handelte es sich um Fahrzeuge des Typs Ford Focus 1 Turnier (Kombi). Die beiden Fahrzeuge wiesen eine Gesamtmasse von 1.297 (Versuch 1) bzw. $1.259 \mathrm{~kg}$ (Versuch 2) auf. Darüber hinaus waren die beiden Fahrzeuge jeweils mit einem Dummy (Typ Hybrid II) mit einer Masse von 75 kg besetzt.

Bei den zu den Crashversuchen herangezogenen Motorrädern handelte es sich um Naked-Bikes des Typs Kawasaki ER5 mit einem Hubraum von ca. $500 \mathrm{~cm}^{3}$. Die Krafträder waren jeweils mit einem Dummy (Typ Hybrid II) mit einer Masse von $75 \mathrm{~kg}$ besetzt und wiesen eine Fahrzeugmasse von 183 kg (Versuch 1) bzw. 186 kg (Versuch 2) auf.

Nachfolgend sind die wesentlichen Parameter des Crashversuches 1 wiedergegeben:

- Ausgangsgeschwindigkeit Pkw sowie Krad: ca. 67 km/h

- Kollisionsgeschwindigkeit Krad: ca. 65 km/h

- Kollisionsgeschwindigkeit Pkw: ca. 32 km/h

- Kollisionsbedingter Geschwindigkeitsabbau Krad: ca. 37 km/h

- Geschwindigkeit Krad nach Kollision: ca. 28 km/h

Die folgenden Abbildungen beschreiben beispielhaft den Versuch 1. 


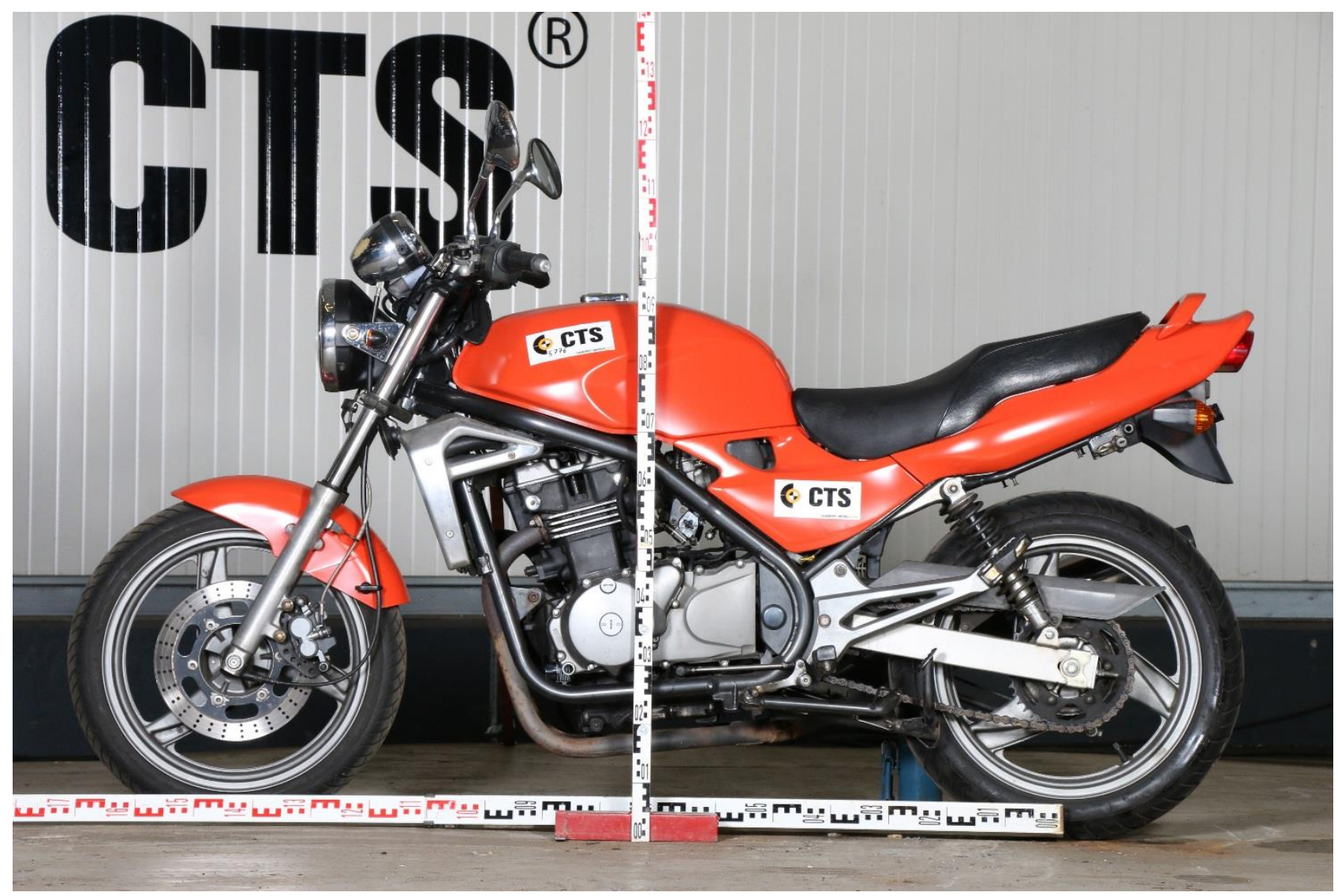

Abbildung 7:

Für den Crashversuch 1 genutztes Kraftrad im unbeschädigten Zustand

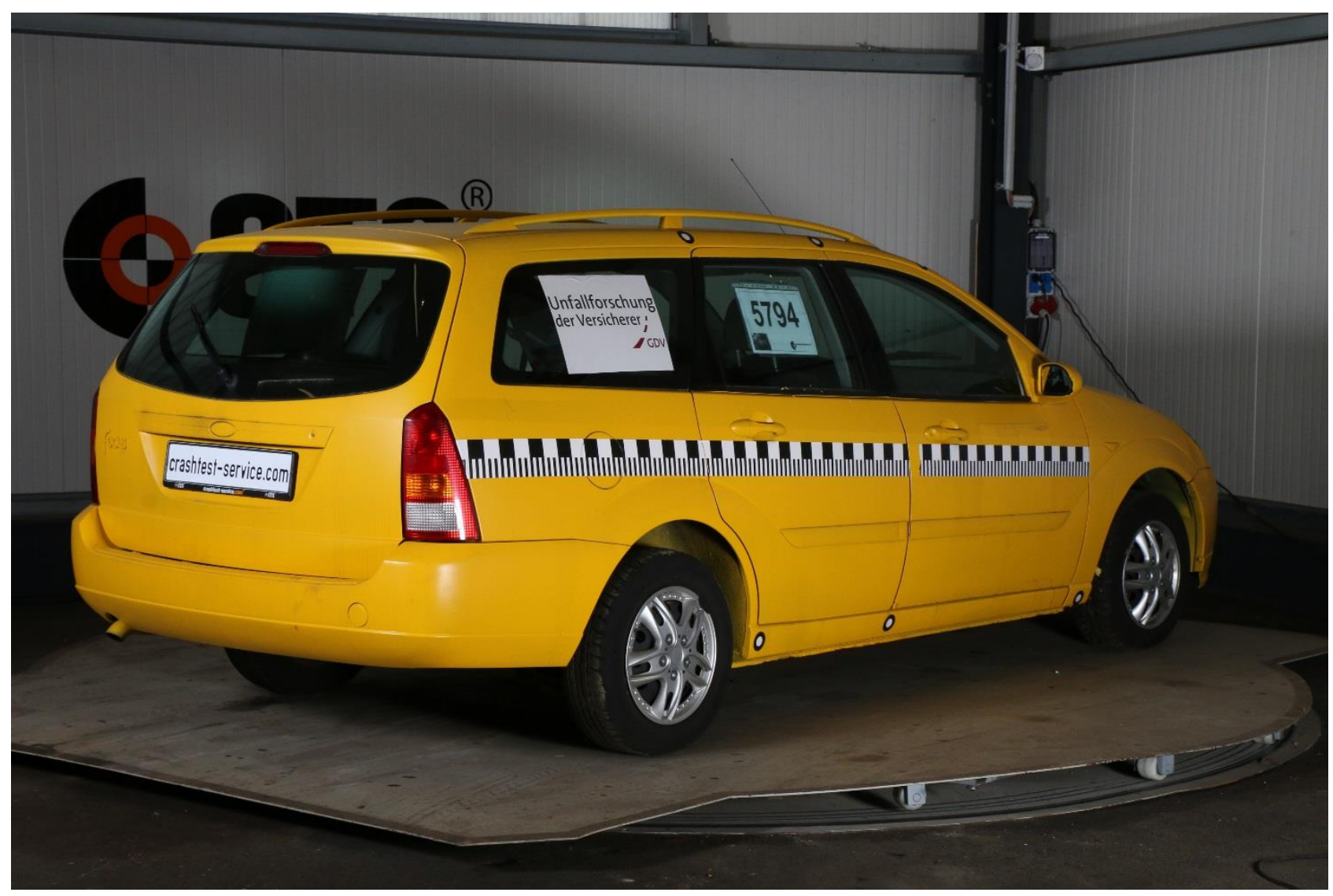

Abbildung 8:

Für den Crashversuch genutzter Pkw 


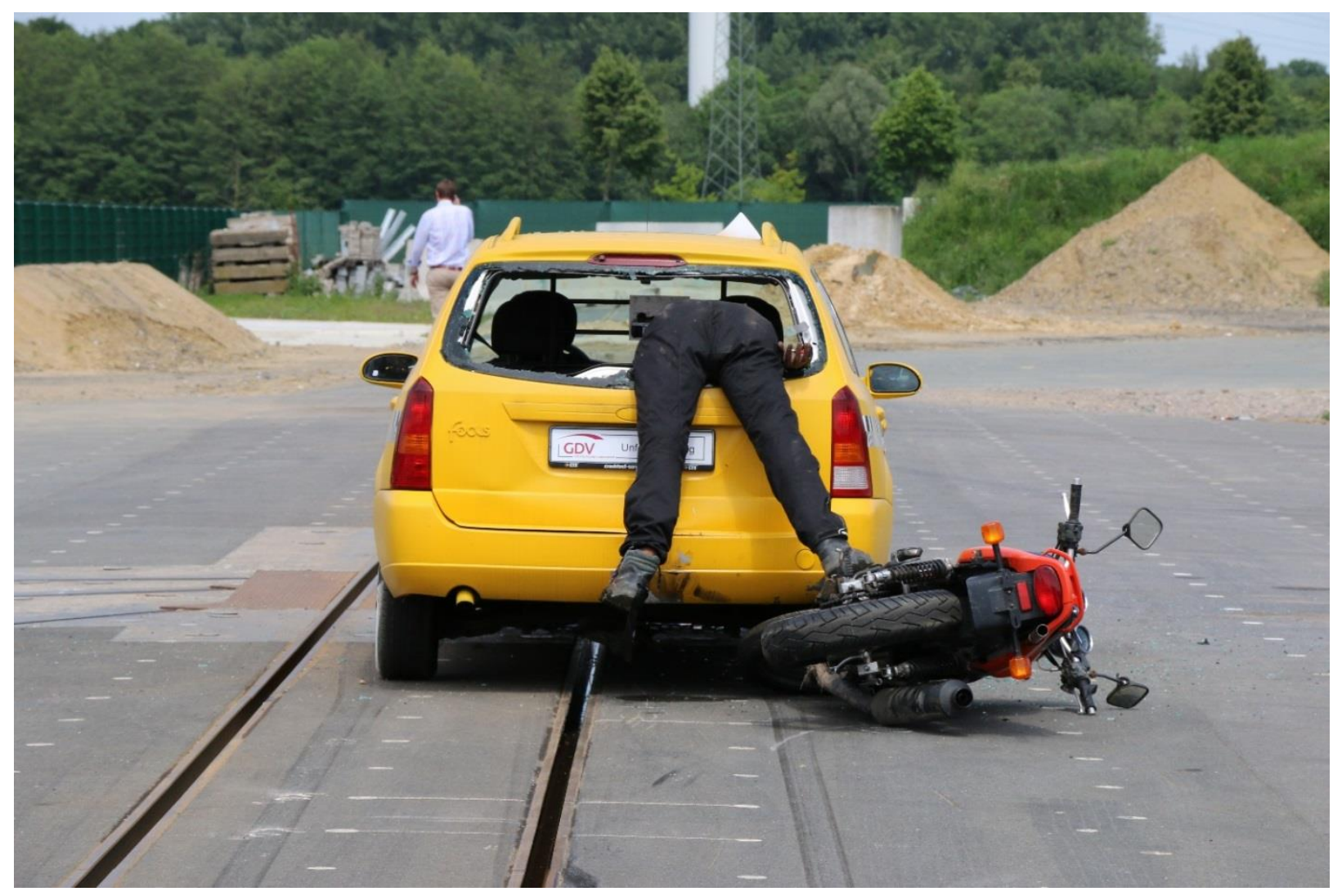

Abbildung 9:

Endstand des Pkw sowie Endlagen von Kraftrad und Kradfahrer nach Versuch 1

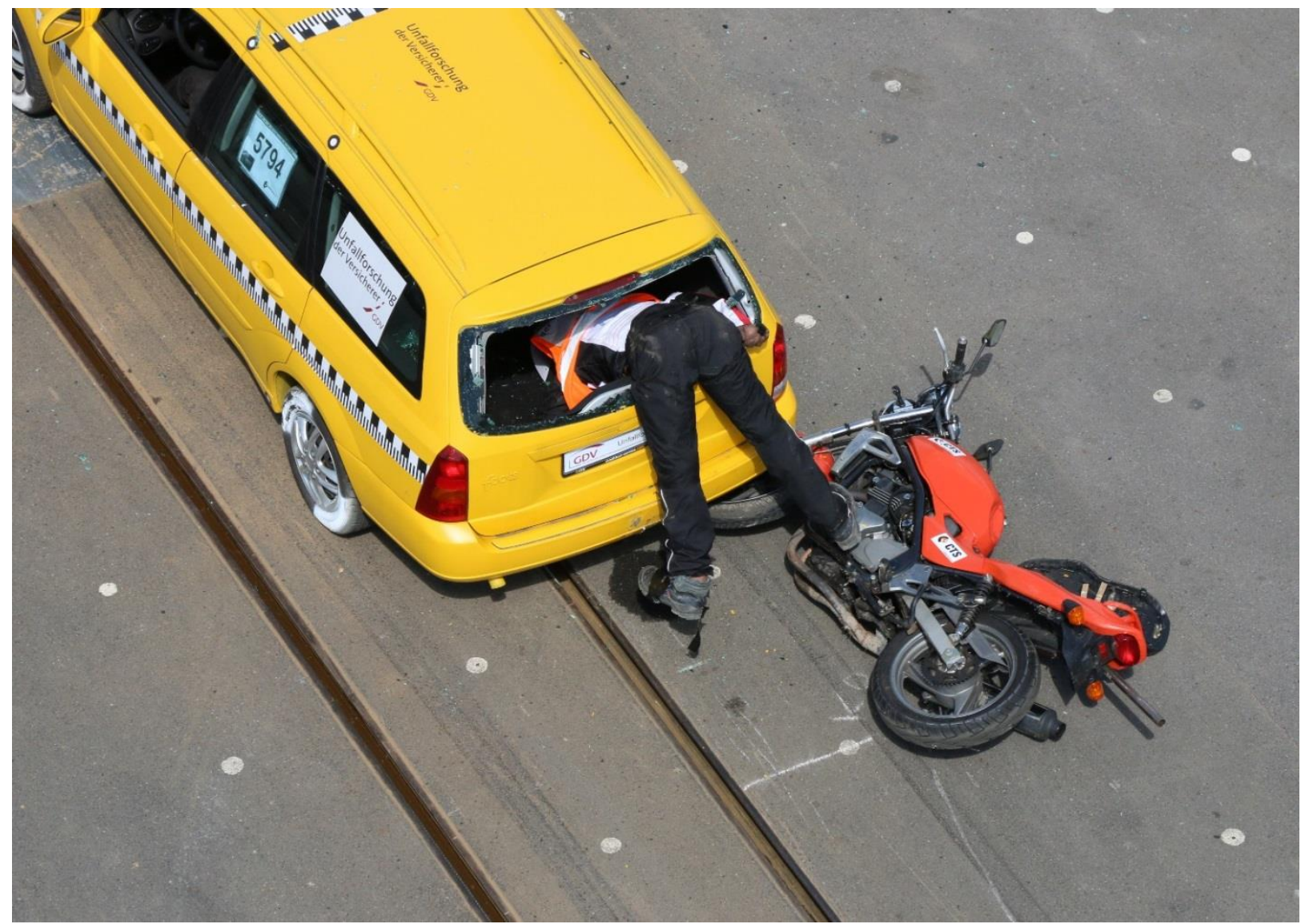

Abbildung 10:

Endstand des Pkw sowie Endlagen von Kraftrad und Kradfahrer nach Versuch 1 aus erhöhter Perspektive 
Nachfolgend sind die wesentlichen Parameter des Crashversuches 2 wiedergegeben:

- Ausgangsgeschwindigkeit Pkw sowie Krad: ca. 65 km/h

- Kollisionsgeschwindigkeit Krad: ca. 63 km/h

- Kollisionsgeschwindigkeit Pkw: ca. 29 km/h

- Kollisionsbedingter Geschwindigkeitsabbau Krad: ca. 32 km/h

- Geschwindigkeit Krad nach Kollision: ca. 31 km/h

Die folgenden Abbildungen beschreiben beispielhaft den Versuch 2.

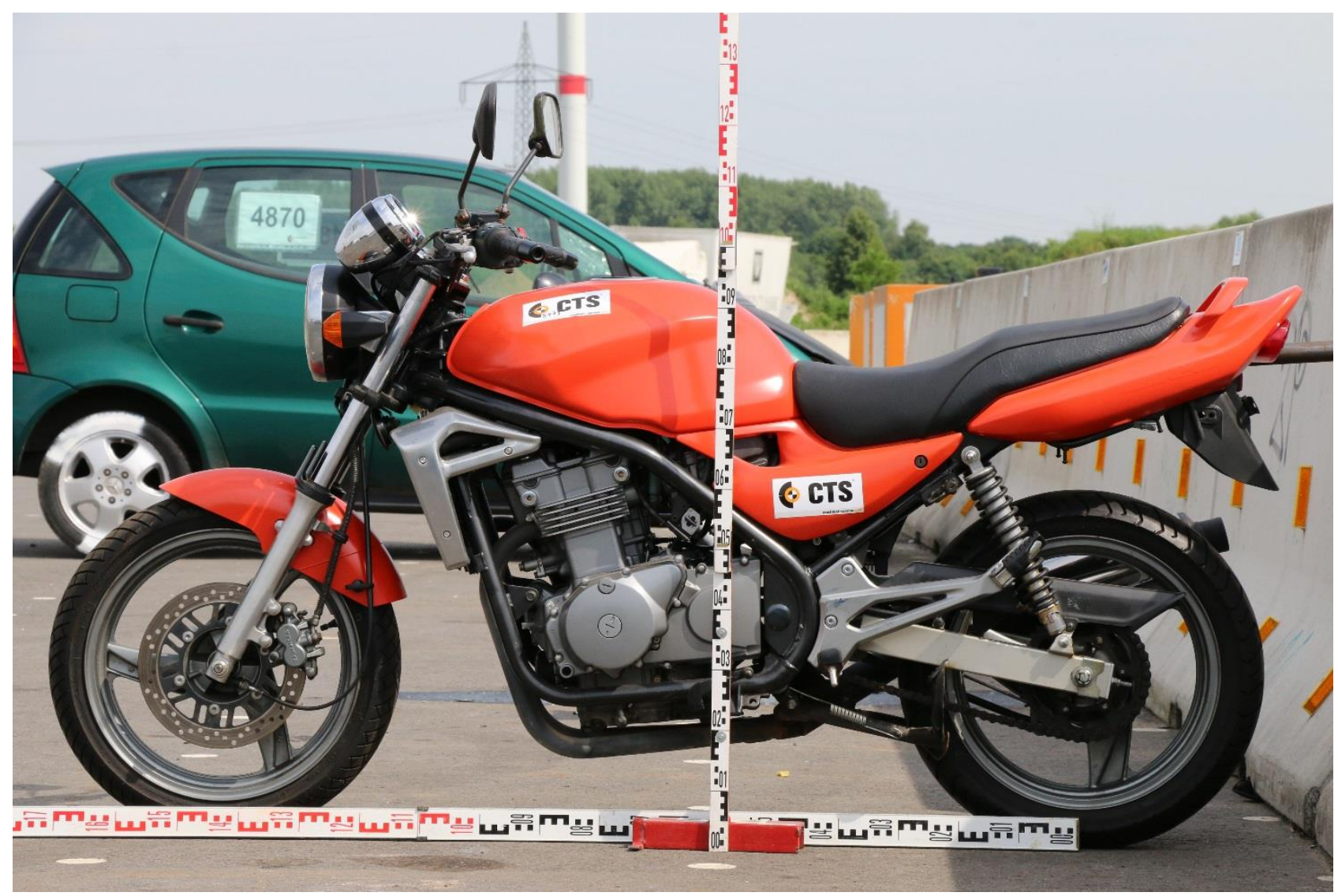

Abbildung 11:

Für den Crashversuch 2 genutztes Kraftrad in Übersicht 


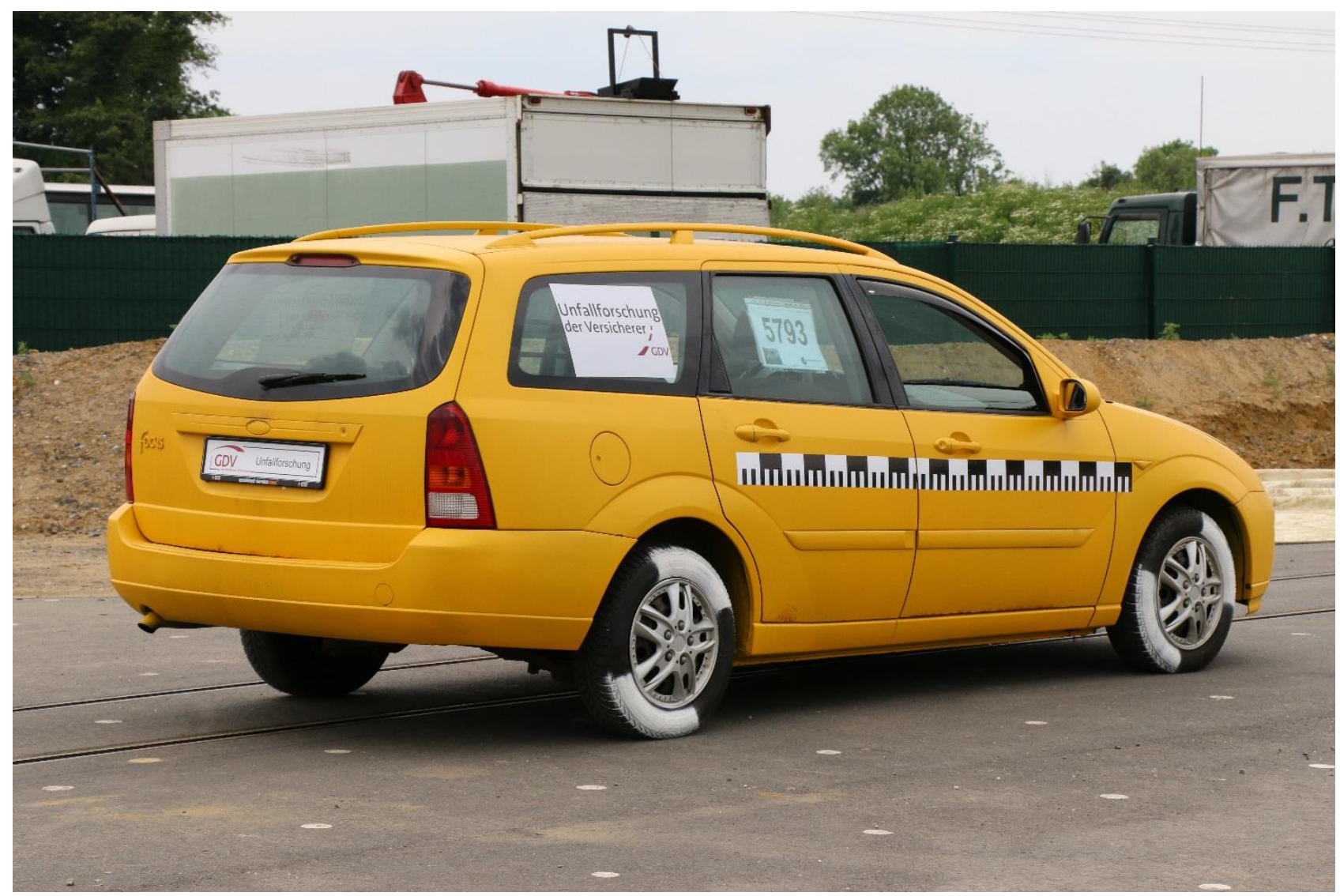

Abbildung 12:

Pkw zur Durchführung des Crashversuchs 2

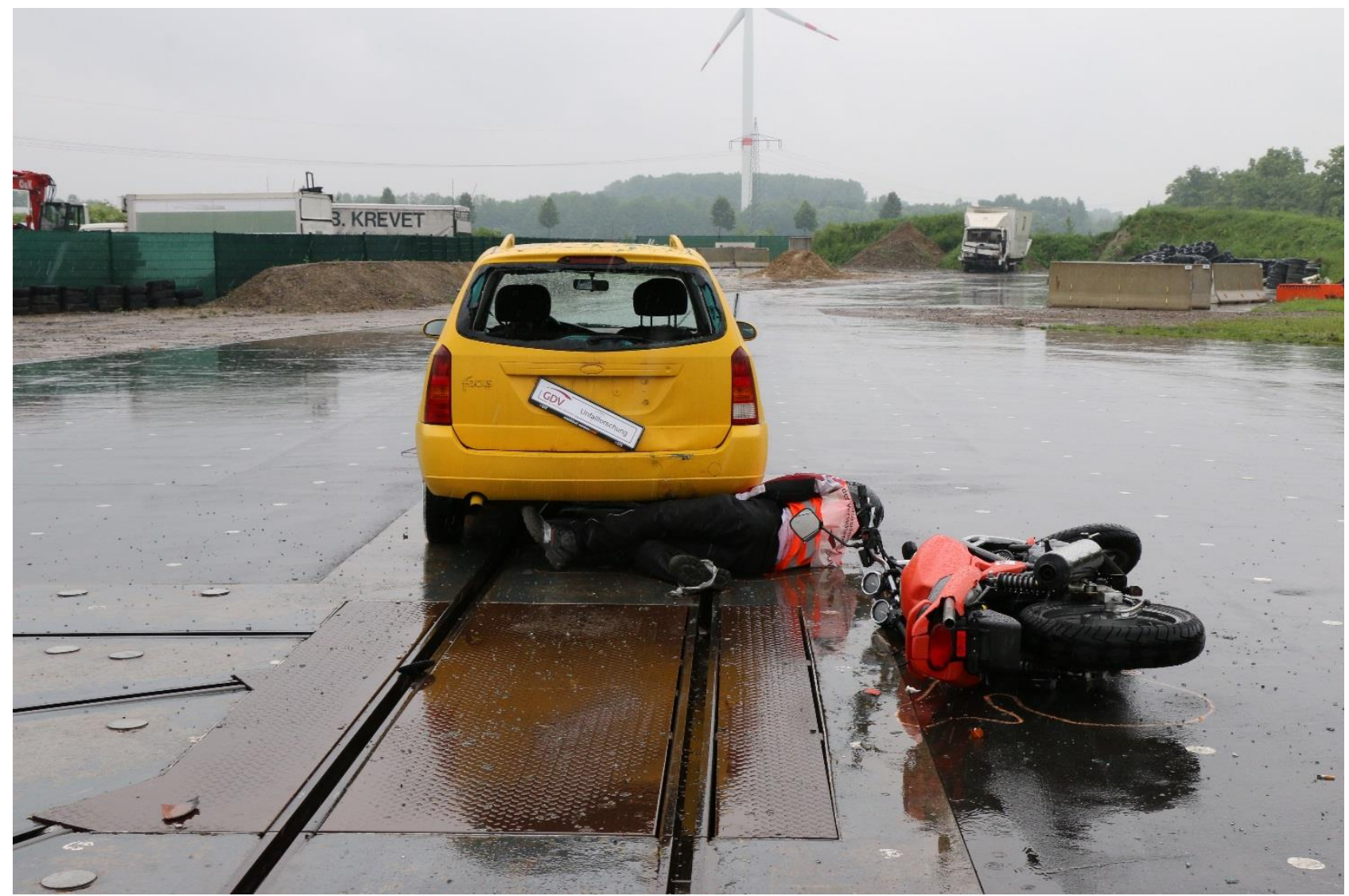

Abbildung 13:

Endlagen des Kraftrades und des Kradfahrers sowie Endstand des Pkw nach der Kollision 


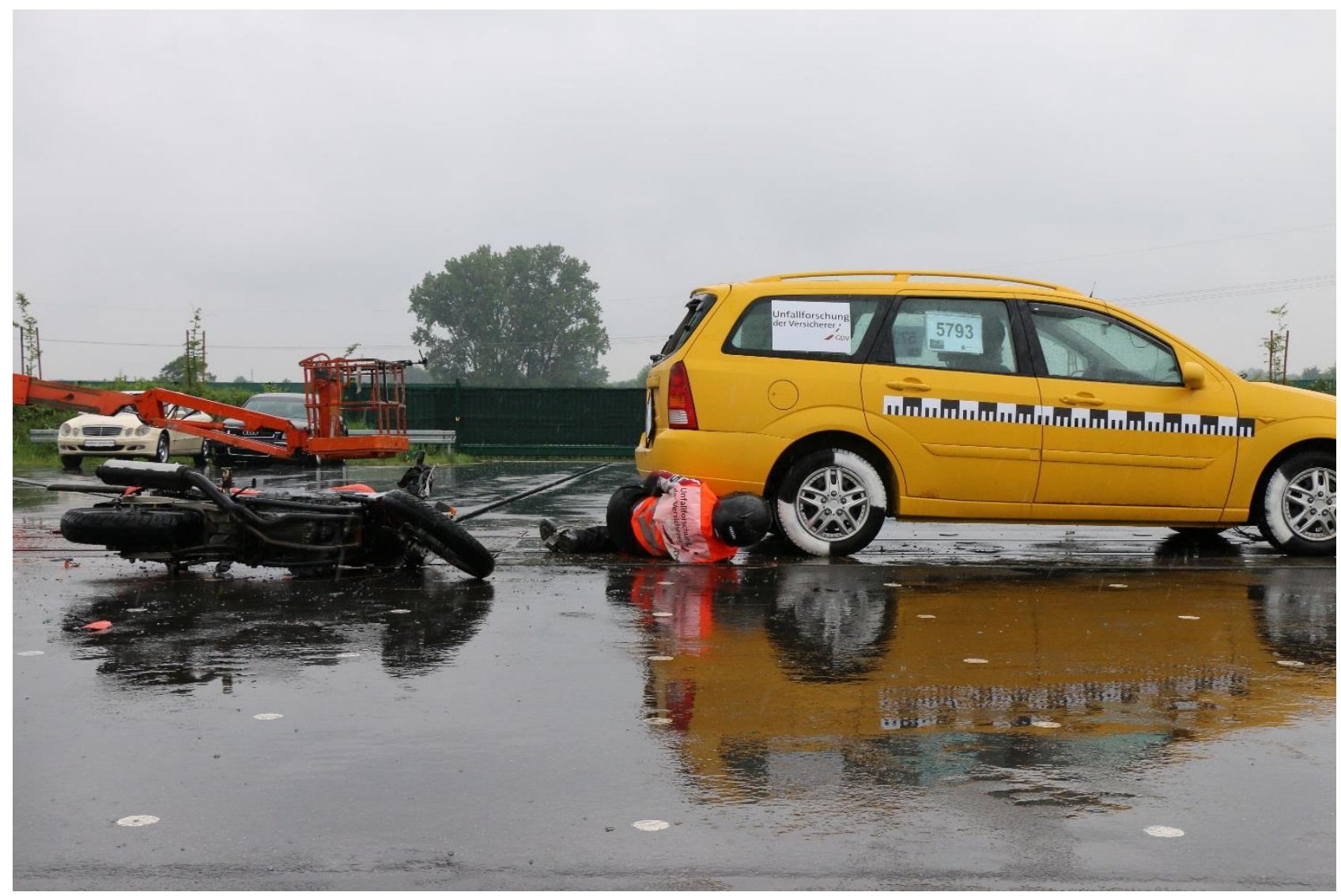

Abbildung 14:

Endlagen des Kraftrades und des Kradfahrers sowie Endstand des Pkw nach der Kollision aus geänderter Perspektive

Die durchgeführten Crashversuche verdeutlichen die hohe Gefahr, die von Auffahrunfällen auch in bereits relativ niedrigen Geschwindigkeitsniveaus ausgeht. Werden die Bewegungsabläufe in der Kollisionsphase und hiermit auch die wirkenden Beschleunigungen bzw. Verzögerungen berücksichtigt, so kann hier von schweren bzw. schwersten Verletzungen des Kradfahrers ausgegangen werden.

Für weitere Details zu den durchgeführten Crashversuchen wird ergänzend auf die in der Anlage eingefügten Datenblätter verwiesen (Versuch 1 - Anlage 2, Versuch 2 - Anlage 3). 


\section{Literatur}

[1] Statistisches Bundesamt, Fachserie 8, Reihe 7, 2013, erschienen am 2. Juli 2014

[2] Statistisches Bundesamt, Verkehrsunfälle, Zweiradunfälle im Straßenverkehr 2013, Wiesbaden, 2014.

[3] EU-Richtlinie 2002/24/EG des europäischen Parlaments und des Rates zur Typengenehmigung für zweirädrige oder dreirädrige Kraftfahrzeuge und zur Aufhebung der Richtlinie 92/61/EWG des Rates, Amtsblatt der Europäischen Gemeinschaft, L 124/1, 9.5.2002

[4] Fahrerlaubnis-Verordnung, FeV vom 13. Dezember 2010 (BGBI. I S. 1980) in der Fassung des Inkrafttretens vom 01.05.2014. Letzte Änderung durch: Zehnte Verordnung zur Änderung der Fahrerlaubnis-Verordnung und anderer straßenverkehrsrechtlicher Vorschriften vom 16. April 2014 (Bundesgesetzblatt Jahrgang 2014 Teil I Nr. 15 S. 348, ausgegeben zu Bonn am 23. April 2014)

[5] Kraftfahrt-Bundesamt, Der Fahrzeugbestand im Überblick am 1. Januar 2014 gegenüber 1. Januar 2013, http://www.kba.de/DE/Statistik/Fahrzeuge/Bestand/Ueberblick/2014_b_ueberblick_pdf.pdf? blob=publicationFile \&v=7

[6] Kraftfahrt-Bundesamt, Bestand an Kraftfahrzeugen und Kraftfahrzeuganhängern am 1. Januar 2014 nach Bundesländern und Fahrzeugklassen, http://www.kba.de/DE/Statistik/Fahrzeuge/Bestand/FahrzeugklassenAufbauarten/2014_b_f zkl_eckdaten_absolut.html?nn=652402

[7] Landespolizeipräsidium des Saarlandes, statistische Erhebungen, unveröffentlicht, 2012

[8] Association for the Advancement of Automotive Medicine The Abbreviated Injury ScaleRevision 1990 Update 98, Association for the Advancement of Automotive Medicine AAAM, Barrington, Illinois, USA, http://www.aaam.org

[9] Statistisches Bundesamt, Fachserie 1, Reihe 1.3, 2011, erschienen am 15.05.2013

[10] Statistisches Bundesamt, Fachserie 3, Reihe 5.1, 2013, erschienen am 18.12.2014

[11] Statistische Ämter des Bundes und der Länder, Verkehr - Straßen des überörtlichen Verkehrs, 05.09.2014

[12] Baker SP, O'Neill B, Haddon W, Long WB (1974): The Injury Severity Score: A method for describing patients with multiple injuries and evaluating emergency care. J Trauma 14: 187 $-197$

[13] Unfallforschung der Versicherer, Intelligente Systeme zur Verbesserung der Motorradsicherheit, Unfallforschung kompakt Nr. 45, Berlin, 2014.

[14] http://www.udv.de/de/initiativen-aktionen/unfallkommission/sicherheitspreis-dieunfallkommission-2013

[15] ADAC (Allgemeiner Deutscher Automobilclub) - Zahlen, Fakten, Wissen, Aktuelles aus dem Verkehr, Ausgabe 2014 


\section{Anlage 1}

Kfz-Bestand, Kfz-Fahrleistungen

\begin{tabular}{|l|r|r|r|r|r|r|r|r|r|r|r|r|}
\hline \multicolumn{1}{|l|}{ Kraftfahrzeuge } & 2003 & 2004 & 2005 & 2006 & 2007 & $2008 *$ & 2009 & 2010 & 2011 & 2012 & 2013 & 2014 \\
\hline & 200 & & & & & & & & & & & \\
\hline $\begin{array}{l}\text { Kfz-Bestand } \\
\text { zum 1.1. in Mio. }\end{array}$ & 53,66 & 54,08 & 54,52 & 54,91 & 55,51 & 49,33 & 49,60 & 50,18 & 50,9 & 51,7 & 52,4 & 53,0 \\
\hline \begin{tabular}{l} 
Pkw-Bestand \\
\multicolumn{1}{|c|}{ davon Diesel }
\end{tabular} & 44,66 & 45,02 & 45,38 & 46,09 & 46,57 & 41,18 & 41,32 & 41,74 & 42,3 & 42,9 & 43,4 & 43,9 \\
\hline Krafträder & 3,65 & 3,72 & 9,1 & 10,10 & 10,80 & 10,00 & 10,03 & 10,82 & 11,3 & 11,9 & 13,0 & 13,2 \\
\hline $\begin{array}{l}\text { Mopeds+Mofas } \\
\text { Kraftomnibusse }\end{array}$ & 1,58 & 1,66 & 1,79 & 1,80 & 1,93 & 1,98 & 2,19 & 2,10 & 2,04 & 2,10 & 2,10 & 2,04 \\
\hline $\begin{array}{l}\text { und Obusse } \\
\text { Lastkraftwagen }\end{array}$ & 0,09 & 0,09 & 0,09 & 0,08 & 0,08 & 0,08 & 0,08 & 0,08 & 0,08 & 0,08 & 0,08 & 0,08 \\
\hline $\begin{array}{l}\text { Sattel- } \\
\text { zugmaschinen }\end{array}$ & 2,18 & 2,59 & 2,57 & 2,57 & 2,60 & 2,32 & 2,35 & 2,39 & 2,44 & 2,53 & 2,58 & 2,63 \\
\hline
\end{tabular}

* ab 2008 ohne vorübergehend abgemeldete Kfz

\begin{tabular}{|l|r|r|r|r|r|r|r|r|r|r|r|r|r|r|r|r|}
\hline \multicolumn{10}{|c|}{ Kraftfahrzeugverkehr - Fahrleistungen nach Kraftfahrzeugarten in Mrd. Fahrzeug-Kilometer } \\
\hline & 2001 & 2002 & 2003 & 2004 & 2005 & 2006 & 2007 & 2008 & 2009 & 2010 & 2011 & 2012 \\
\hline Kfz insgesamt & 682,7 & 687,3 & 682,2 & 696,4 & 684,3 & 687,3 & 692,0 & 690,1 & 699,0 & 704,8 & 717,6 & 719,3 \\
\hline Mopeds & 4,1 & 3,8 & 3,9 & 4,2 & 4,3 & 4,6 & 4,6 & 4,7 & 4,8 & 4,7 & 4,8 & 4,8 \\
\hline Krafträder & 13,8 & 12,2 & 12,5 & 12,7 & 13,0 & 13,2 & 10,8 & 11,1 & 11,4 & 11,6 & 11,9 & 12,1 \\
\hline $\begin{array}{l}\text { Personen- } \\
\text { kraftwagen }\end{array}$ & 575,5 & 583,6 & 577,8 & 590,4 & 578,2 & 583,9 & 587,5 & 584,6 & 595,0 & 599,0 & 608,8 & 610,1 \\
\hline Kraftomnibusse & 3,7 & 3,6 & 3,6 & 3,6 & 3,5 & 3,5 & 3,4 & 3,3 & 3,4 & 3,3 & 3,3 & 3,3 \\
\hline Lastkraftwagen & 60,2 & 58,2 & 57,9 & 57,7 & 57,0 & 57,6 & 59,8 & 60,3 & 59,5 & 60,7 & 62,5 & 63,2 \\
\hline Sattelzugmasch. & 13,7 & 13,7 & 14,0 & 15,1 & 15,5 & 16,6 & 17,8 & 18,0 & 16,5 & 16,9 & 17,5 & 16,8 \\
\hline Sonstige Kfz & 11,7 & 12,3 & 12,4 & 12,6 & 12,8 & 7,8 & 8,0 & 8,1 & 8,4 & 8,5 & 8,8 & 9,0 \\
\hline $\begin{array}{l}\text { Bundes- } \\
\text { autobahnen }\end{array}$ & 207,4 & 211,3 & 213,1 & 218,9 & 216,2 & 218,7 & 225,3 & 221,4 & 226,9 & 217,1 & 223,2 & 222,0 \\
\hline
\end{tabular}

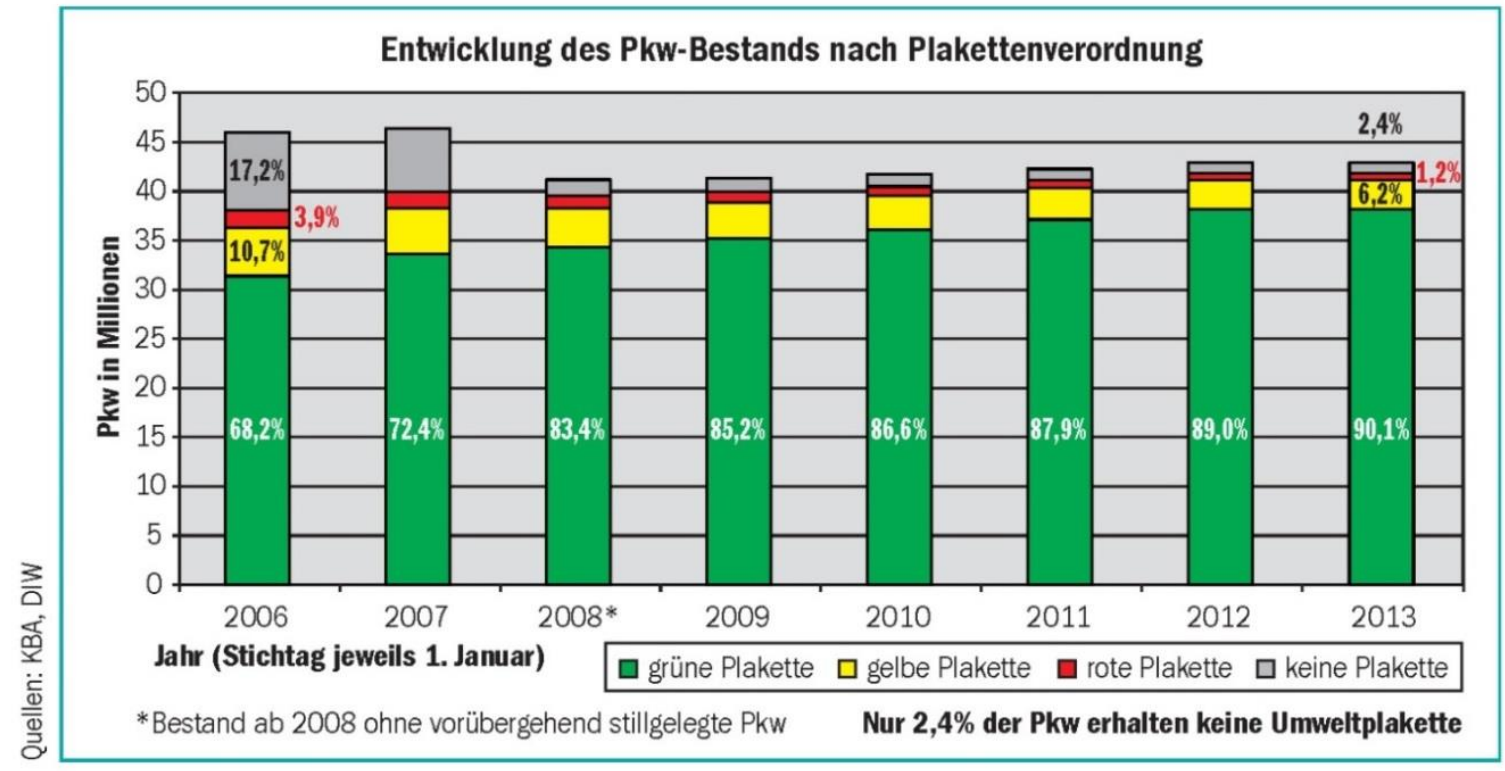




\section{Anlage 1}

10

\section{Verunglückte nach Art der Verkehrsbeteiligung}

\begin{tabular}{|c|c|c|c|c|c|c|c|c|}
\hline \multirow{3}{*}{ Jahr } & \multicolumn{8}{|c|}{ Verunglïckte } \\
\hline & \multirow[t]{2}{*}{ insgesamt } & \multicolumn{6}{|c|}{ Fahrer und Mitfahrer von } & \multirow{2}{*}{$\begin{array}{l}\text { Fuß- } \\
\text { gänger }\end{array}$} \\
\hline & & $\begin{array}{l}\text { Mofas/ } \\
\text { Mopeds }\end{array}$ & $\begin{array}{l}\text { Motor- } \\
\text { rädern }\end{array}$ & $\begin{array}{c}\text { Personen- } \\
\text { kraft- } \\
\text { wagen }\end{array}$ & $\begin{array}{l}\text { Kraftomni- } \\
\text { bussen, } \\
\text { Obussen }\end{array}$ & $\begin{array}{l}\text { Güter- } \\
\text { kraftfahr- } \\
\text { zeugen }\end{array}$ & $\begin{array}{l}\text { Fahr- } \\
\text { rädern }\end{array}$ & \\
\hline 1980 & 555.966 & 61.951 & 54.896 & 296.261 & 5.162 & 11.639 & 54.583 & 67.809 \\
\hline 1990 & 521.977 & 22.272 & 42.506 & 318.423 & 5.361 & 10.160 & 69.294 & 50.067 \\
\hline 2000 & 511.577 & 19.373 & 41.112 & 313.892 & 5.077 & 14.900 & 73.397 & 38.989 \\
\hline 2005 & 438.804 & 17.864 & 35.703 & 250.114 & 5.241 & 12.201 & 78.434 & 34.602 \\
\hline 2007 & 436.368 & 21.498 & 34.802 & 244.377 & 5.252 & 12.200 & 79.004 & 34.499 \\
\hline 2008 & 413.524 & 22.209 & 30.640 & 227.123 & 5.005 & 11.149 & 79.423 & 33.423 \\
\hline 2009 & 401.823 & 19.914 & 30.370 & 223.508 & 5.235 & 10.354 & 75.797 & 32.238 \\
\hline 2010 & 374.818 & 17.247 & 26.969 & 213.396 & 5.580 & 11.539 & 65.573 & 30.139 \\
\hline 2011 & 396.374 & 18.679 & 30.680 & 217.238 & 5.736 & 10.595 & 76.655 & 32.111 \\
\hline 2012 & 387.978 & 17.344 & 27.947 & 216.068 & 5.671 & 10.053 & 74.463 & 31.830 \\
\hline $2013^{*}$ & 377.500 & 15.225 & 27.334 & 212.543 & 5.818 & 9.948 & 71.403 & 31.350 \\
\hline
\end{tabular}

\begin{tabular}{|c|c|c|c|c|c|c|c|c|}
\hline \multirow{3}{*}{ Jahr } & \multicolumn{8}{|c|}{ Tödlich Verunglüickte } \\
\hline & \multirow[t]{2}{*}{ insgesamt } & \multicolumn{6}{|c|}{ Fahrer und Mitfahrer von } & \multirow{2}{*}{$\begin{array}{l}\text { Fuß- } \\
\text { gänger }\end{array}$} \\
\hline & & $\begin{array}{l}\text { Mofas/ } \\
\text { Mopeds }\end{array}$ & $\begin{array}{l}\text { Motor- } \\
\text { rädern }\end{array}$ & $\begin{array}{c}\text { Personen- } \\
\text { kraft- } \\
\text { wagen }\end{array}$ & $\begin{array}{c}\text { Kraftomni- } \\
\text { bussen, } \\
\text { Obussen }\end{array}$ & $\begin{array}{c}\text { Güter- } \\
\text { kraftfahr- } \\
\text { zeugen }\end{array}$ & $\begin{array}{l}\text { Fahr- } \\
\text { rädern }\end{array}$ & \\
\hline 1980 & 15.050 & 1.056 & 1.575 & 6.915 & 49 & 269 & 1.338 & 3.720 \\
\hline 1990 & 11.046 & 384 & 1.059 & 6.256 & 23 & 211 & 908 & 2.113 \\
\hline 2000 & 7.503 & 157 & 945 & 4.396 & 9 & 274 & 659 & 992 \\
\hline 2005 & 5.361 & 107 & 875 & 2.833 & 9 & 208 & 575 & 686 \\
\hline 2007 & 4.949 & 100 & 807 & 2.625 & 26 & 213 & 425 & 695 \\
\hline 2008 & 4.477 & 110 & 656 & 2.368 & 10 & 180 & 456 & 653 \\
\hline 2009 & 4.152 & 99 & 650 & 2.110 & 12 & 164 & 462 & 591 \\
\hline 2010 & 3.648 & 74 & 635 & 1.840 & 32 & 162 & 381 & 476 \\
\hline 2011 & 4.009 & 70 & 708 & 1.986 & 10 & 169 & 399 & 614 \\
\hline 2012 & 3.600 & 93 & 586 & 1.791 & 3 & 152 & 406 & 520 \\
\hline $2013^{*}$ & 3.340 & 73 & 568 & 1.588 & 11 & 148 & 354 & 556 \\
\hline
\end{tabular}

* vorläufige Werte
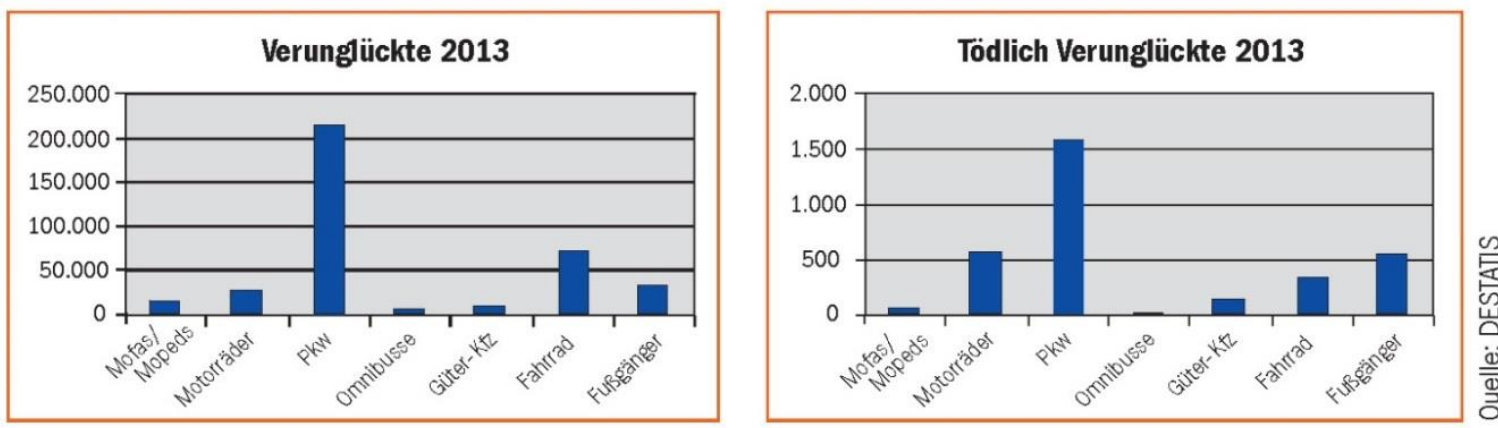


\section{Anlage 1}

Unfallrisiko

\begin{tabular}{|l|r|r|r|r|}
\hline \multirow{2}{*}{$\begin{array}{l}\text { Entwicklung des Risikos, } \\
\text { im Straßenverkehr }\end{array}$} & \multicolumn{2}{|c|}{ Verunglïcktenrate } & \multicolumn{2}{|c|}{ Getötetenrate } \\
\cline { 2 - 5 } zu verunglïcken & \multicolumn{1}{|c|}{ Verunglückte pro 10 Mio. Pkm } & \multicolumn{2}{|c|}{ Getötete pro 1 Mrd. Pkm } \\
\hline Krafträder & 1990 & 2012 & 1990 & 2012 \\
\hline Mopeds & 48,7 & 21,4 & 112,7 & 44,8 \\
\hline Fußgänger & 43,5 & 33,5 & 58,9 & 18,0 \\
\hline Fahrradfahrer & 17,4 & 9,2 & 62,4 & 15,1 \\
\hline Sattelzugmaschinen & 35,8 & 21,2 & 39,3 & 11,6 \\
\hline Pkw & 1,3 & 0,8 & 4,1 & 2,6 \\
\hline Lkw & 4,4 & 2,5 & 6,9 & 2,1 \\
\hline Omnibusse & 1,8 & 1,1 & 2,7 & 1,3 \\
\hline
\end{tabular}
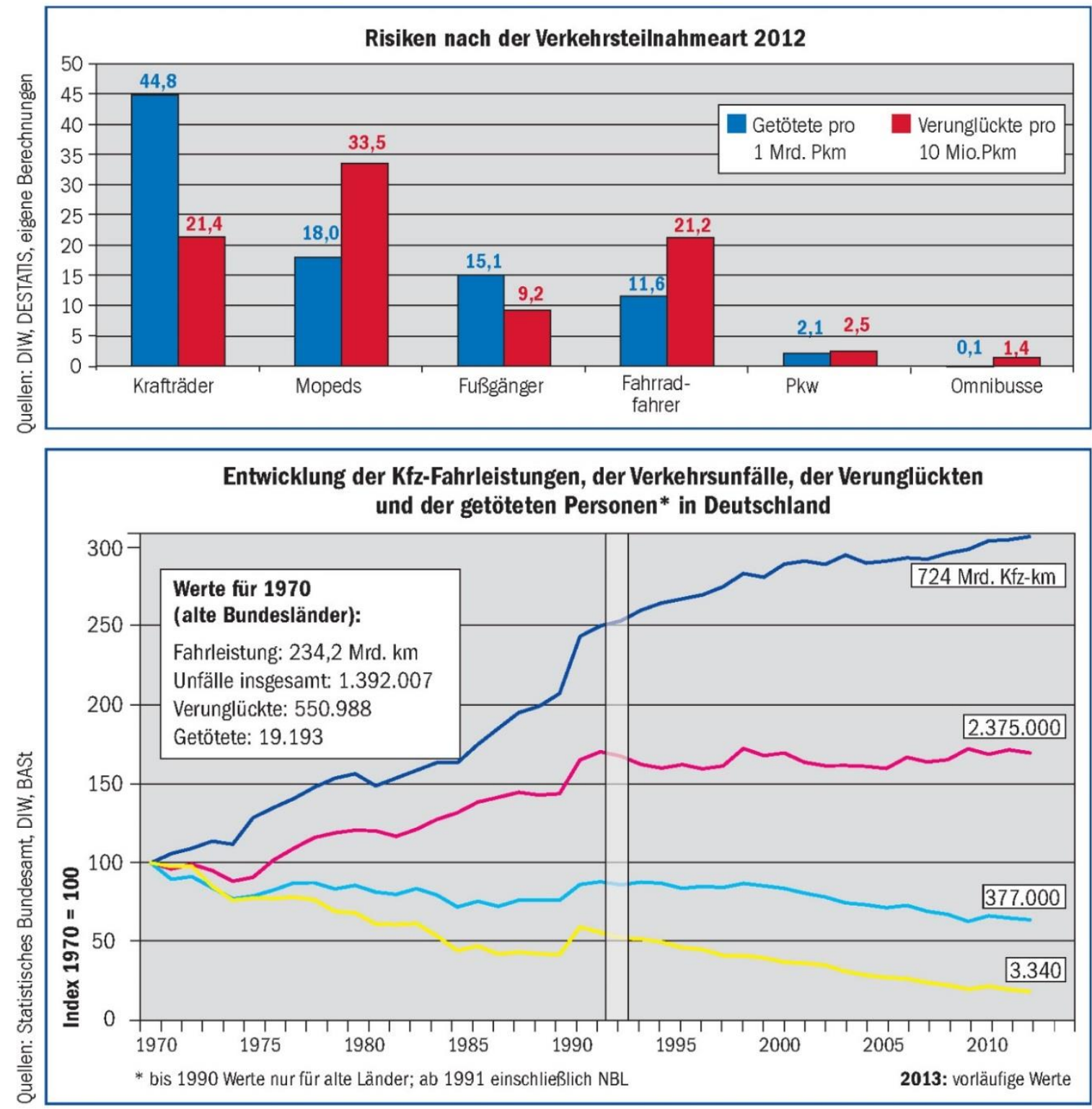


\section{Anlage 2}

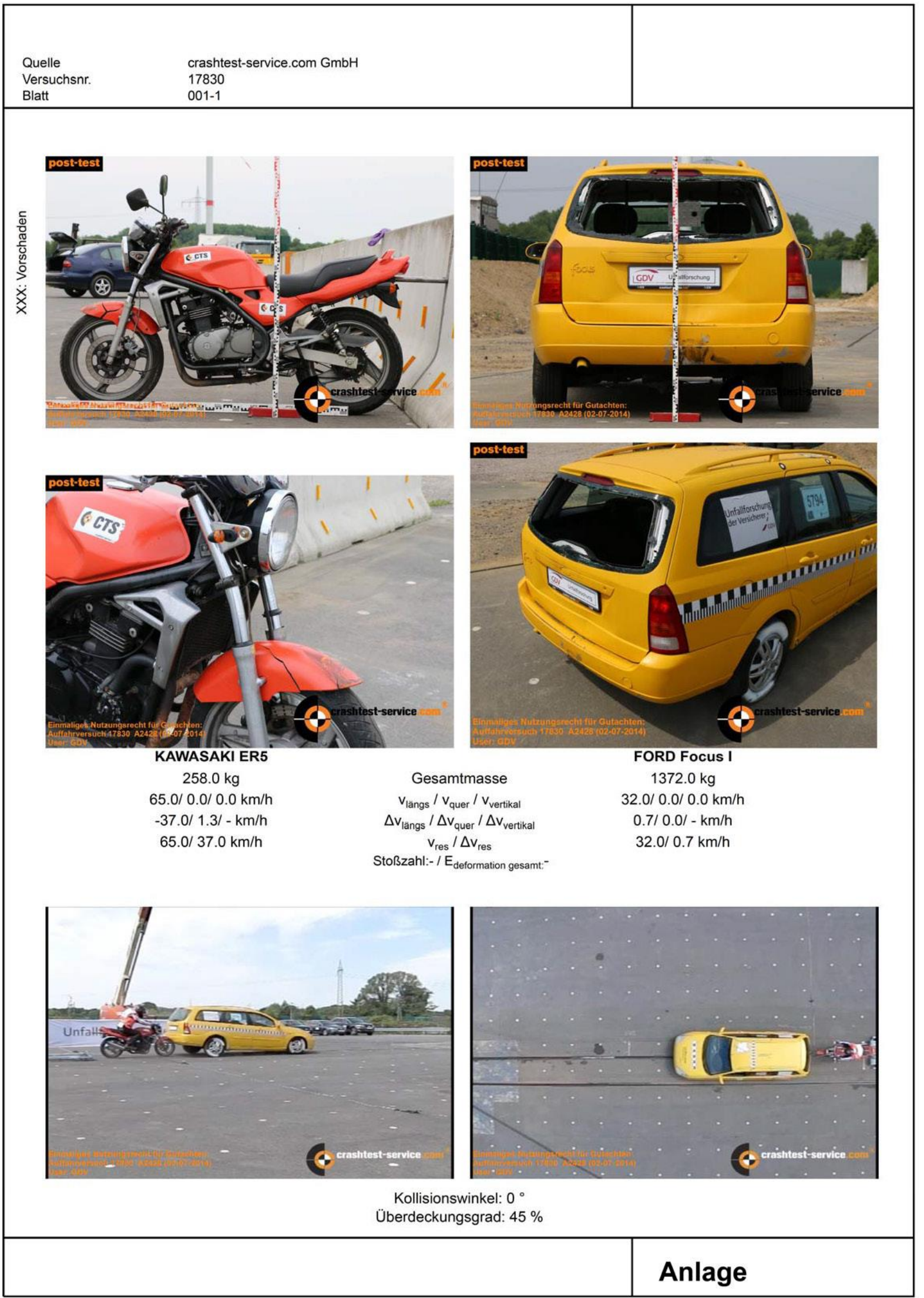


Anlage 2

Quelle

Versuchsnr.

crashtest-service.com GmbH

Blatt

17830

001-2

Allgemeine Versuchsdaten

Versuchsdatum:

13.05.2014

Anstoßhöhe:

Schalldruckpegel

Messung 1 (außen)

Messung 2 (außen):

Mehrfahrzeugkollision:

Besonderheiten:

Mess-Abstand: -

Mess-Abstand: -

KAWASAKI fährt mit ca. $65 \mathrm{~km} / \mathrm{h}$ auf Heck des ca. $32 \mathrm{~km} / \mathrm{h}$ schnellen,

vollverzögertem FORD auf. Vrel ca.33km/h. Vorversuch zu 17845

Bemerkungen: 


\section{Anlage 3}

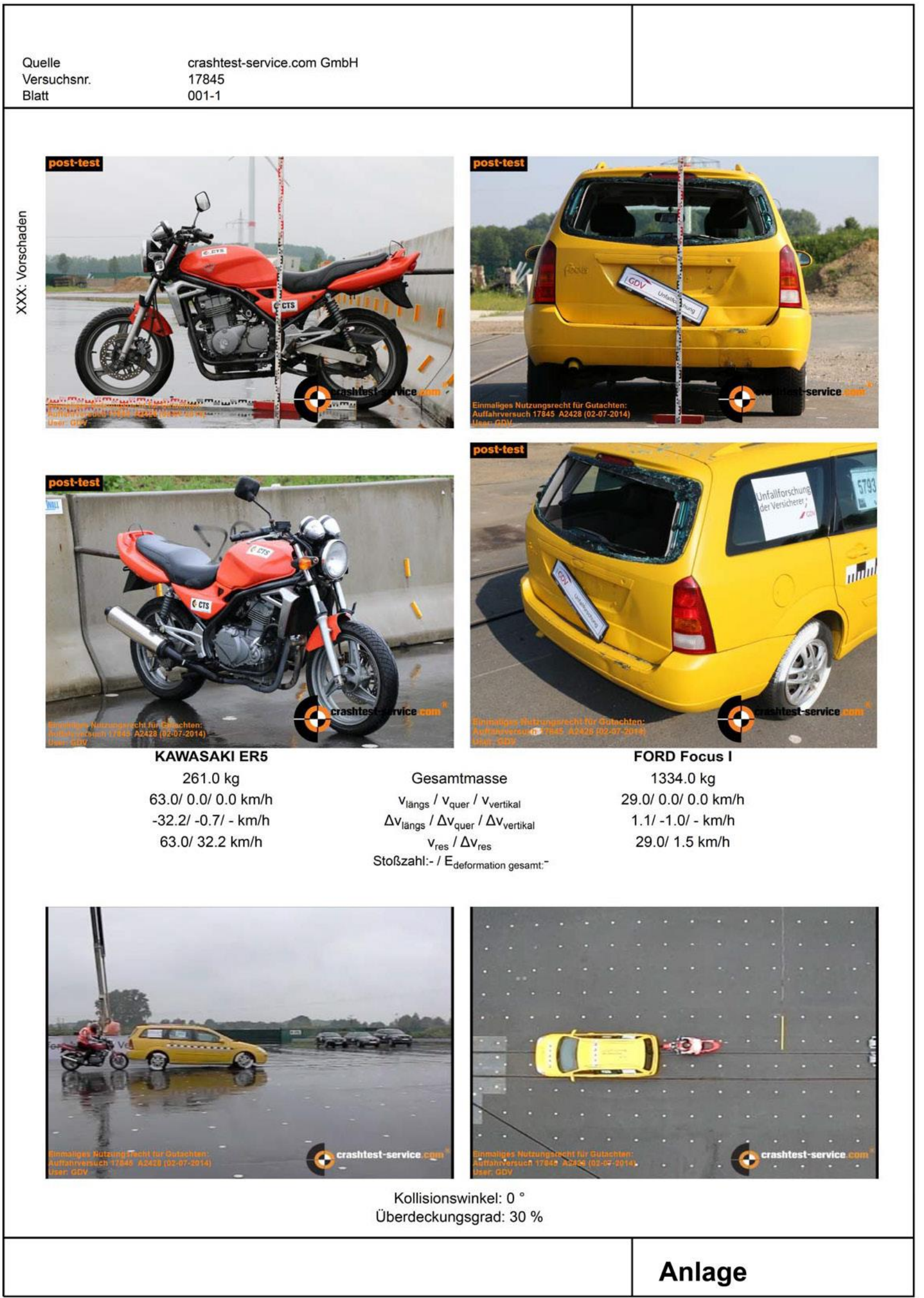


Anlage 3

Quelle

Versuchsnr.

crashtest-service.com $\mathrm{GmbH}$

Blatt

17845

$001-2$

Allgemeine Versuchsdaten

Versuchsdatum:

27.05.2014

Anstoßhöhe:

Schalldruckpegel

Messung 1 (außen)

Messung 2 (außen):

Mehrfahrzeugkollision:

Besonderheiten:

Mess-Abstand: -

Mess-Abstand: -

KAWASAKI fährt mit ca. $63 \mathrm{~km} / \mathrm{h}$ auf Heck des ca. $29 \mathrm{~km} / \mathrm{h}$ schnellen, vollverzögertem FORD auf. Vrel ca. $34 \mathrm{~km} / \mathrm{h}$.

Bemerkungen: 


\section{GDV}

DIE DEUTSCHEN VERSICHERER

\section{Gesamtverband der Deutschen Versicherungswirtschaft e. V.}

Wilhelmstraße 43 / 43 G, 10117 Berlin

Postfach 0802 64, 1002 Berlin

Tel. $030 / 2020$-50 00, Fax 030 / 2020 - 6000

www.gdv.de, www.udv.de 